\title{
Diastereoselective Mannich Reactions using Fluorinated Ketones: Synthesis of Stereogenic Carbon-Fluorine Units
}

\author{
Ya Li,* Xiang Li, Huaqi Shang, Xiangyu Chen, Xinfeng Ren \\ Department of Chemistry and Chemical Engineering, Shanghai University of \\ Engineering Science, 333 Longteng Road, Shanghai, 201620 \\ E-mail:ya.li@sues.edu.cn
}

\section{Contents}

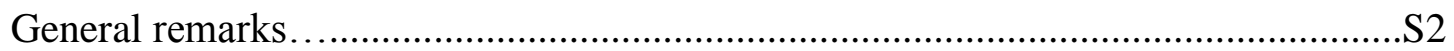

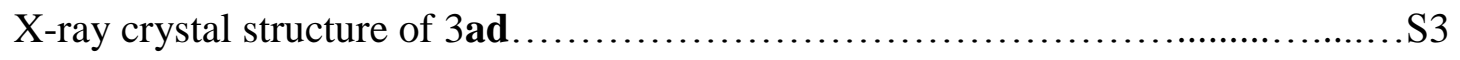

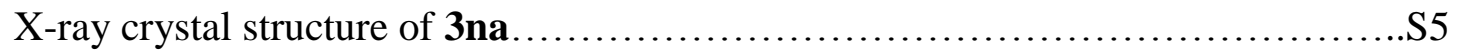

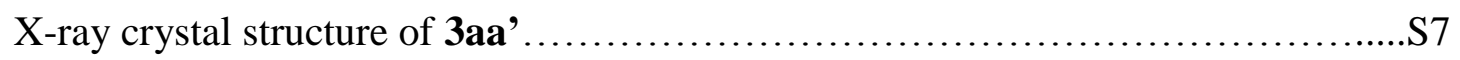

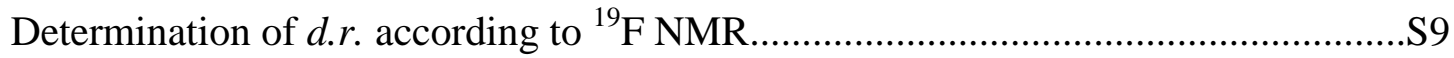

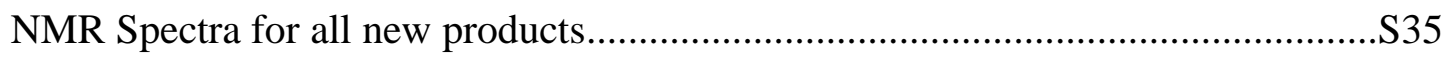




\section{General Remarks}

Unless otherwise mentioned, all commercial reagents and solvents were used directly as purchased. Compounds 2 were prepared according to literature methods. ${ }^{[1-2]}$ The ether was dried with molecular sieves before use. Flash chromatography was performed on silica gel with petroleum ether/ethyl acetate as the eluent. Melting points were uncorrected. Optical rotations were measured with a sodium lamp. ${ }^{1} \mathrm{H}$, ${ }^{13} \mathrm{C}$ and ${ }^{19} \mathrm{~F}$ NMR spectra were recorded on a $400 \mathrm{MHz}$ NMR spectrometer. High-resolution mass data were recorded on a high-resolution mass spectrometer in the ESI mode. Chemical shifts $(\delta)$ are reported in parts per million and referenced to the residual solvent peak, and $J$ values are given in hertz $(\mathrm{Hz})$. 


\section{X-ray crystal structure of $3 \mathrm{ad}$}

The thermal ellipsoids are drawn at a $30 \%$ probability level.<smiles>CC(C)(C)S(=O)N[C@H](c1ccc(Cl)cc1)C1(F)CCc2ccccc2C1=O</smiles>

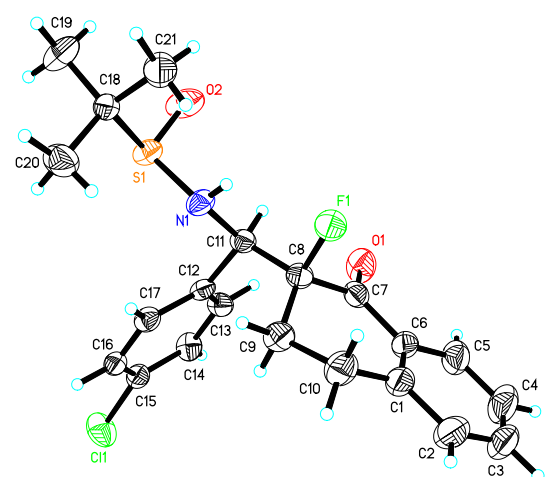

Table 1. Crystal data and structure refinement for 3ad.

\section{Empirical formula}

Formula weight

Temperature

Wavelength

Crystal system

Space group

Unit cell dimensions

Volume

Z

Density (calculated)

Absorption coefficient

$\mathrm{F}(000)$

Crystal size

Theta range for data collection

Index ranges

Reflections collected

Independent reflections

\section{$\mathrm{C} 21 \mathrm{H} 23 \mathrm{Cl}$ F N O2 S}

407.91

293(2) K

$0.71073 \AA$

Monoclinic

P 21

$\mathrm{a}=6.0578(13) \AA \quad \alpha=90^{\circ}$.

$\mathrm{b}=8.7727(18) \AA \quad \beta=94.406(5)^{\circ}$.

$\mathrm{c}=19.173(4) \AA \quad \gamma=90^{\circ}$.

1015.9(4) $\AA^{3}$

2

$1.334 \mathrm{Mg} / \mathrm{m}^{3}$

$0.315 \mathrm{~mm}^{-1}$

428

$0.220 \times 0.180 \times 0.100 \mathrm{~mm}^{3}$

2.131 to $25.497^{\circ}$.

$-7<=\mathrm{h}<=5,-10<=\mathrm{k}<=10,-22<=1<=23$

5841

$3611[\mathrm{R}(\mathrm{int})=0.0329]$ 
Completeness to theta $=25.242^{\circ}$

Absorption correction

Max. and min. transmission

Refinement method

Data / restraints / parameters

Goodness-of-fit on $\mathrm{F}^{2}$

Final R indices [I $>2 \operatorname{sigma}(\mathrm{I})]$

$\mathrm{R}$ indices (all data)

Absolute structure parameter

Extinction coefficient

Largest diff. peak and hole
$99.9 \%$

Semi-empirical from equivalents

0.7456 and 0.6330

Full-matrix least-squares on $\mathrm{F}^{2}$

$3611 / 1 / 251$

0.997

$\mathrm{R} 1=0.0509, \mathrm{wR} 2=0.1083$

$\mathrm{R} 1=0.0720, \mathrm{wR} 2=0.1191$

$0.05(6)$

$\mathrm{n} / \mathrm{a}$

0.278 and -0.135 e. $\AA^{-3}$

Data collection: APEX2 ${ }^{1}$; cell refinement: SAINT $^{1}$; data reduction: SAINT; program(s) used to solve structure: SHELXS97 ${ }^{2}$; program(s) used to refine structure: SHELXL97 $7^{3}$. 


\section{X-ray crystal structure of 3 na}

The thermal ellipsoids are drawn at a 30\% probability level.<smiles>CC(C)(C)C(=O)[C@H](F)[C@H](N[S@@](=O)C(C)(C)C)c1ccccc1</smiles>

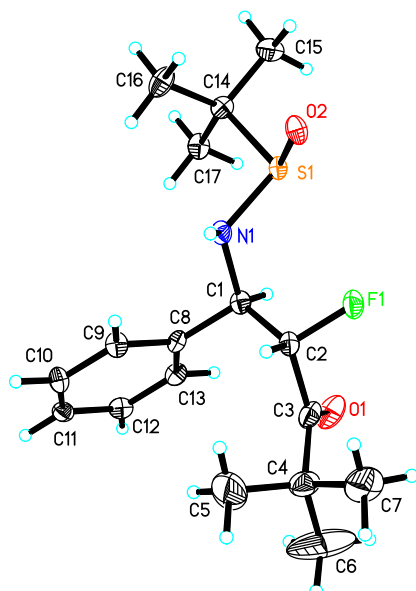

Table 1. Crystal data and structure refinement for 3na.

Empirical formula

Formula weight

Temperature

Wavelength

Crystal system

Space group

Unit cell dimensions

Volume

Z

Density (calculated)

Absorption coefficient

$\mathrm{F}(000)$

Crystal size

Theta range for data collection

Index ranges
C18.33 H29.33 F N O2.33 S

352.15

133(2) K

$0.71073 \AA$

Monoclinic

P 21

$\mathrm{a}=16.297(2) \AA$

$\alpha=90^{\circ}$.

$\mathrm{b}=10.6491(13) \AA$

$\beta=98.884(3)^{\circ}$.

$\mathrm{c}=18.241(2) \AA$

$\gamma=90^{\circ}$.
6

$1.122 \mathrm{Mg} / \mathrm{m}^{3}$

$0.174 \mathrm{~mm}^{-1}$

1140

$0.250 \times 0.200 \times 0.150 \mathrm{~mm}^{3}$

1.821 to $26.000^{\circ}$.

$-17<=\mathrm{h}<=20,-13<=\mathrm{k}<=12,-22<=1<=22$ 
Reflections collected

Independent reflections

Completeness to theta $=25.242^{\circ}$

Absorption correction

Max. and min. transmission

Refinement method

Data / restraints / parameters

Goodness-of-fit on $\mathrm{F}^{2}$

Final R indices [I $>2 \operatorname{sigma}(\mathrm{I})]$

$\mathrm{R}$ indices (all data)

Absolute structure parameter

Largest diff. peak and hole
23046

$12200[\mathrm{R}($ int $)=0.0469]$

$99.9 \%$

Semi-empirical from equivalents

0.7457 and 0.5253

Full-matrix least-squares on $\mathrm{F}^{2}$

12200 / 35 / 671

1.024

$\mathrm{R} 1=0.0537, \mathrm{wR} 2=0.1263$

$\mathrm{R} 1=0.0768, \mathrm{wR} 2=0.1410$

$-0.04(4)$

0.522 and -0.286 e. $\AA^{-3}$

Data collection: $\mathrm{APEX}^{1}$; cell refinement: $\mathrm{SAINT}^{1}$; data reduction: SAINT; program(s) used to solve structure: SHELXS $97^{2}$; $\operatorname{program}(\mathrm{s})$ used to refine structure: SHELXL97 $7^{3}$. 


\section{X-ray crystal structure of 3aa'}

The thermal ellipsoids are drawn at a $30 \%$ probability level.<smiles>CC(C)(C)S(=O)NC(c1ccccc1)C1(F)CCc2ccccc2C1=O</smiles>

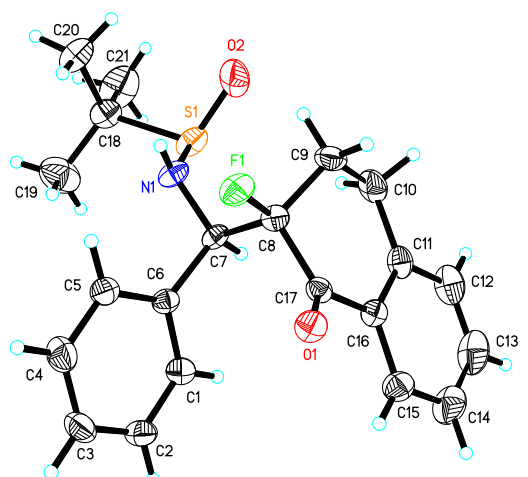

Table 1. Crystal data and structure refinement for 3aa'.

Empirical formula

Formula weight

Temperature

Wavelength

Crystal system

Space group

Unit cell dimensions

Volume

$\mathrm{Z}$

Density (calculated)

Absorption coefficient

$\mathrm{F}(000)$

Crystal size

Theta range for data collection

Index ranges

Reflections collected

Independent reflections

Completeness to theta $=25.242^{\circ}$
C21 H24 F N O2 S

373.47

293(2) K

$0.71073 \AA$

Monoclinic

P 21

$\mathrm{a}=7.926(8) \AA$

$\alpha=90^{\circ}$.

$\mathrm{b}=10.565(10) \AA$

$\beta=102.26(2)^{\circ}$.

$\mathrm{c}=11.841(12) \AA$ $\gamma=90^{\circ}$.

969.0(17) $\AA^{3}$

2

$1.280 \mathrm{Mg} / \mathrm{m}^{3}$

$0.191 \mathrm{~mm}^{-1}$

396

$0.200 \times 0.160 \times 0.100 \mathrm{~mm}^{3}$

1.760 to $25.498^{\circ}$.

$-8<=\mathrm{h}<=9,-12<=\mathrm{k}<=12,-12<=\mathrm{l}<=14$

4627

$3079[\mathrm{R}(\mathrm{int})=0.0509]$

$96.3 \%$ 
Absorption correction

Max. and min. transmission

Refinement method

Data / restraints / parameters

Goodness-of-fit on $\mathrm{F}^{2}$

Final $\mathrm{R}$ indices [I $>2 \operatorname{sigma}(\mathrm{I})]$

$\mathrm{R}$ indices (all data)

Absolute structure parameter

Extinction coefficient

Largest diff. peak and hole
Semi-empirical from equivalents

0.7456 and 0.5848

Full-matrix least-squares on $\mathrm{F}^{2}$

3079 / 2 / 242

1.003

$\mathrm{R} 1=0.0409, \mathrm{wR} 2=0.0808$

$\mathrm{R} 1=0.0618, \mathrm{wR} 2=0.0903$

$0.04(7)$

$\mathrm{n} / \mathrm{a}$

0.201 and -0.277 e. $\AA^{-3}$

Data collection: $\mathrm{APEX}^{1}$; cell refinement: $\mathrm{SAINT}^{1}$; data reduction: SAINT; program(s) used to solve structure: SHELXS97²; program(s) used to refine structure: SHELXL97 ${ }^{3}$.

1. Bruker (2009). APEX2, SAINT and SADABS. Bruker AXS Inc., Madison, Wisconsin, USA.

2. Sheldrick, G. M. (2008). Acta Cryst. A64, 112-122.

3. Sheldrick, G. M. (2015). Acta Cryst. C71, 3-8. 
d.r. Determination by ${ }^{19}$ F NMR on the Crude Products 3.

Crude 3aa, d.r. $=92: 3: 5$<smiles>CC(C)(C)S(=O)N[C@H](c1ccccc1)C1(F)CCc2ccccc2C1=O</smiles>

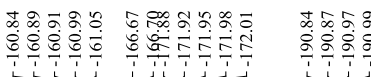

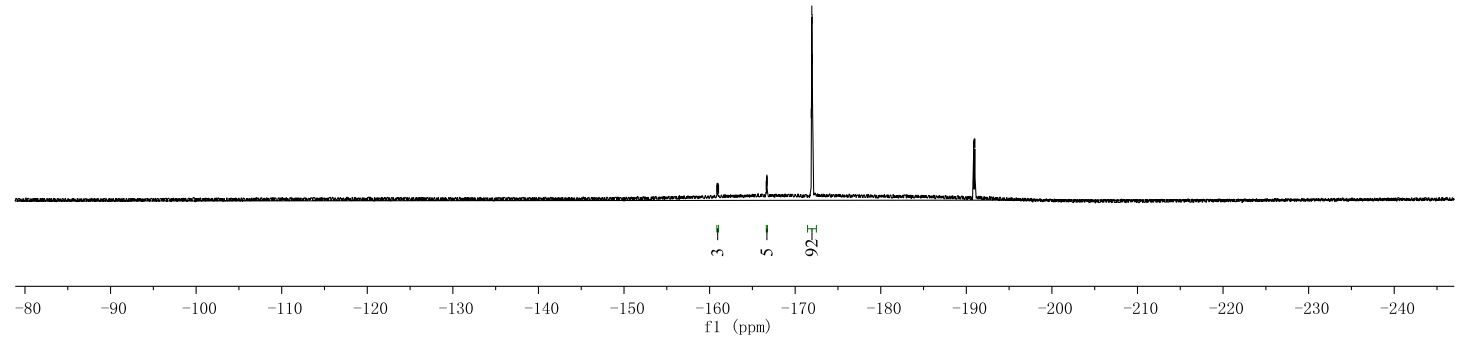

The stereoisomers are at $\delta-160.84--161.04(\mathrm{~m}),-166.69(\mathrm{dd}, J=22.8,12.8 \mathrm{~Hz})$, $-171.44--172.48(\mathrm{~m})$. 
Crude 3ba, d.r. $=94: 6$
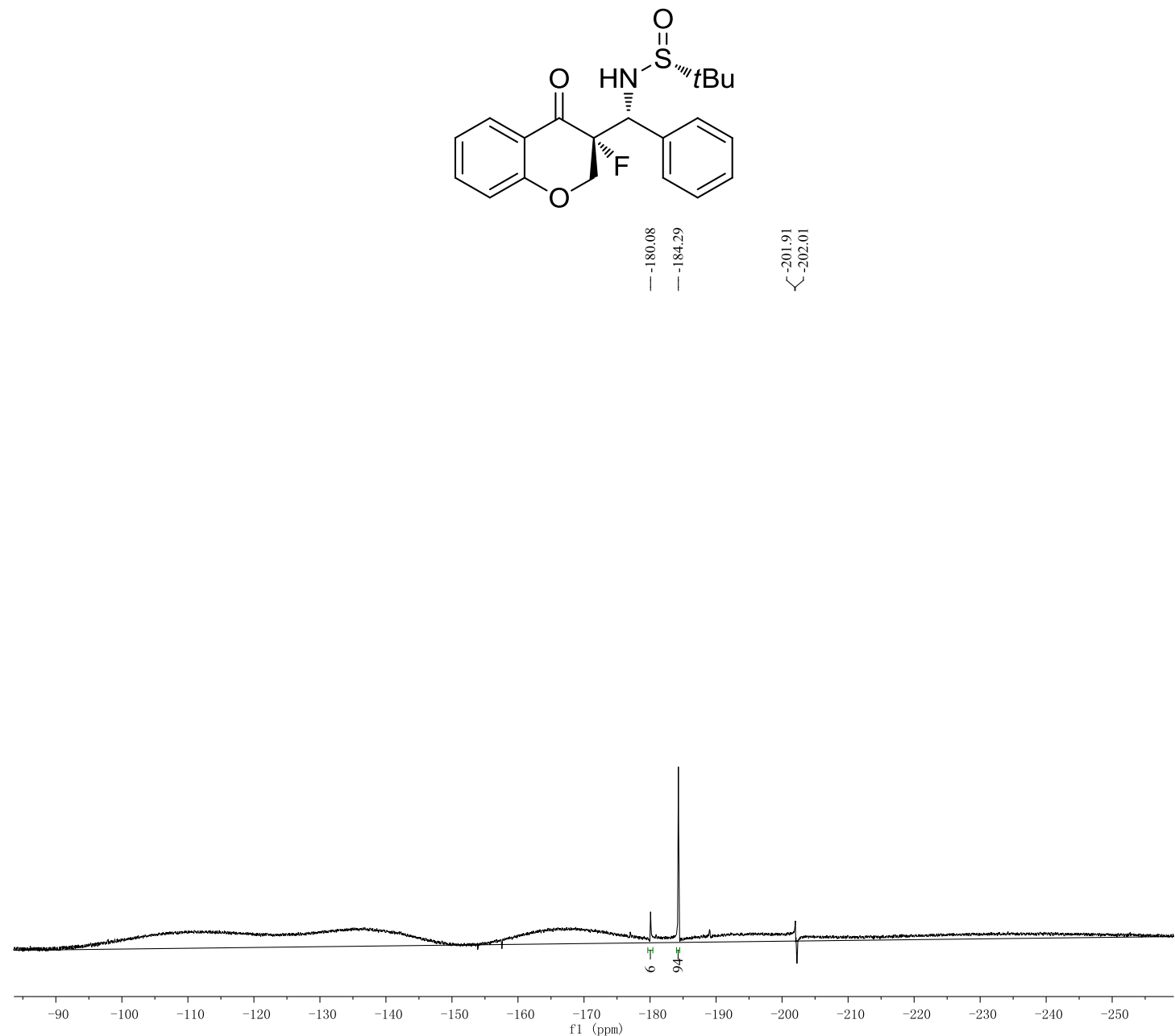

The stereoisomers are at $\delta-180.08(\mathrm{~s}),-184.29$ (s). 
Crude 3ca, d.r. $=91: 9$<smiles>CC(C)(C)S(=O)N[C@H](c1ccccc1)C1(F)CCc2sccc2C1=O</smiles>

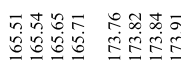

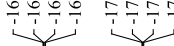

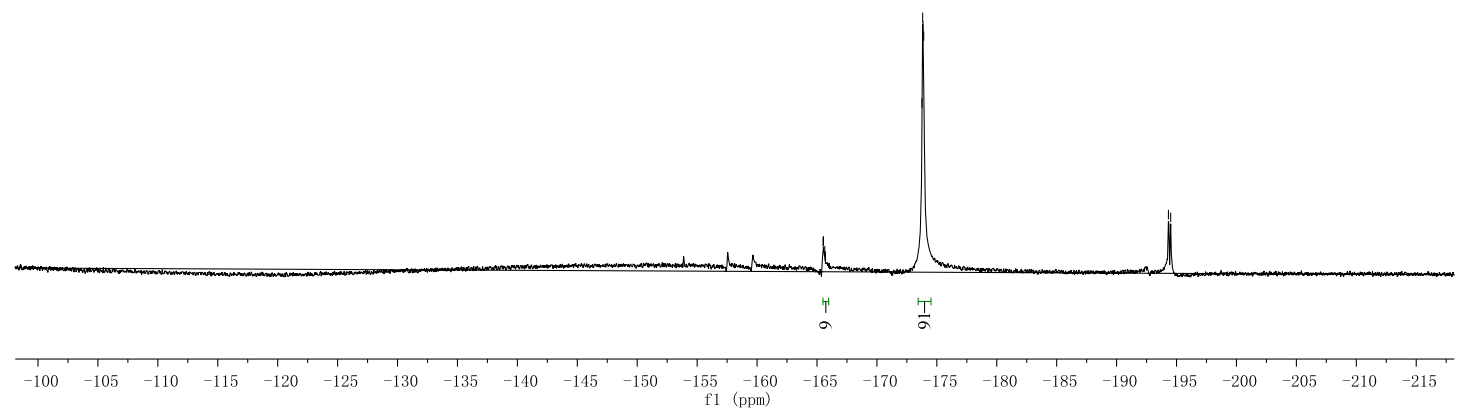

The stereoisomers are at $\delta-165.60(\mathrm{dd}, J=58.9,17.9 \mathrm{~Hz}),-173.83(\mathrm{dd}, J=31.9,23.6$ $\mathrm{Hz})$. 
Crude 3da, d.r. $=92: 8$
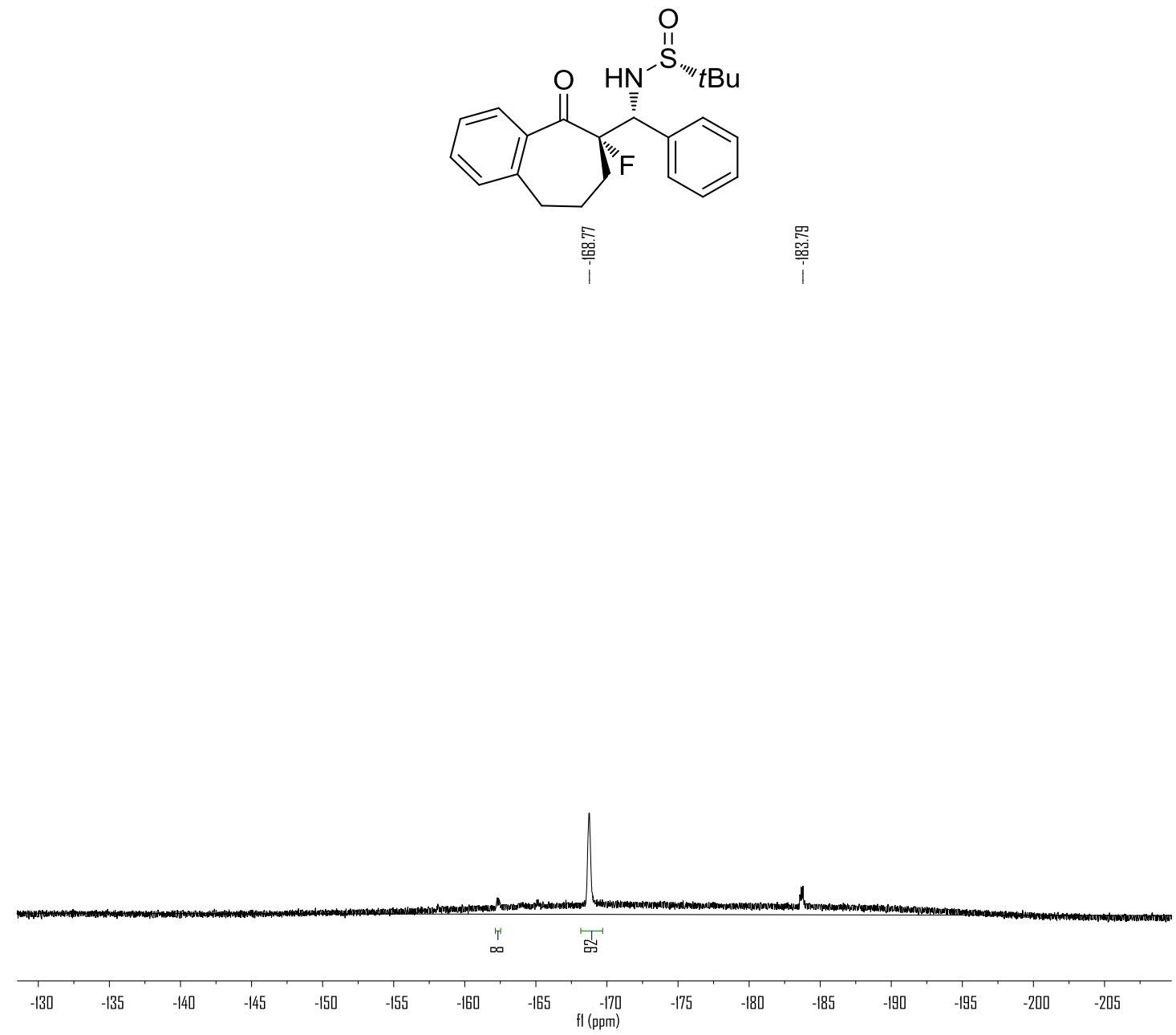

The stereoisomers are at $\delta-162.15--162.52(\mathrm{~m}),-168.77(\mathrm{~s})$. 
Crude 3ea, d.r. $=93: 7$<smiles>CC(C)(C)S(=O)NC(c1ccccc1)C1Cc2ccccc2C1=O</smiles>

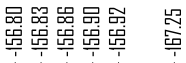

vij

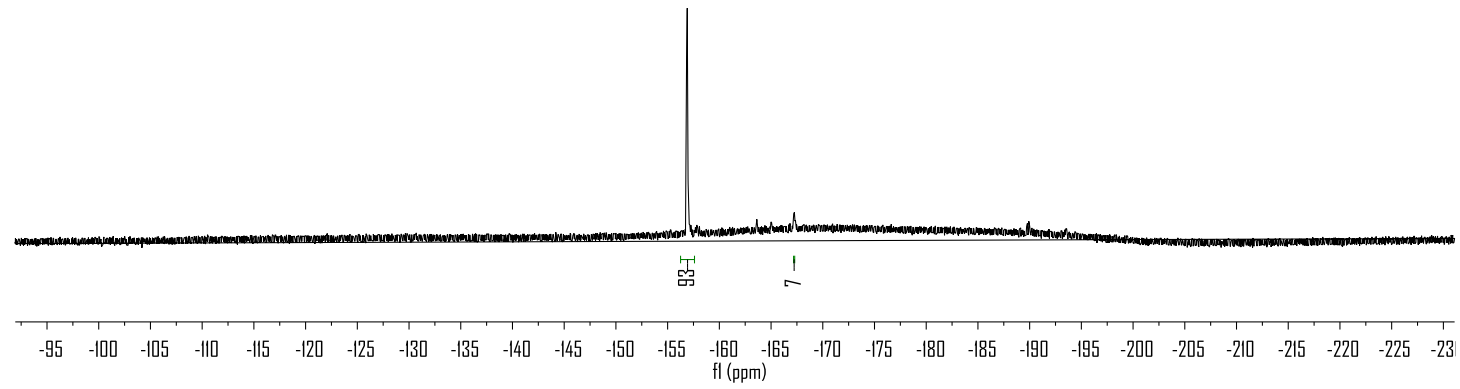

The stereoisomers are at $\delta-156.25--157.60(\mathrm{~m}),-167.25(\mathrm{~s})$. 
Crude 3fa, d.r. $=82: 5: 6: 7$<smiles>CC(C)(C)S(=O)N[C@H](c1ccccc1)C1(F)C(=O)Cc2ccccc21</smiles>

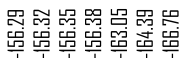

i i i

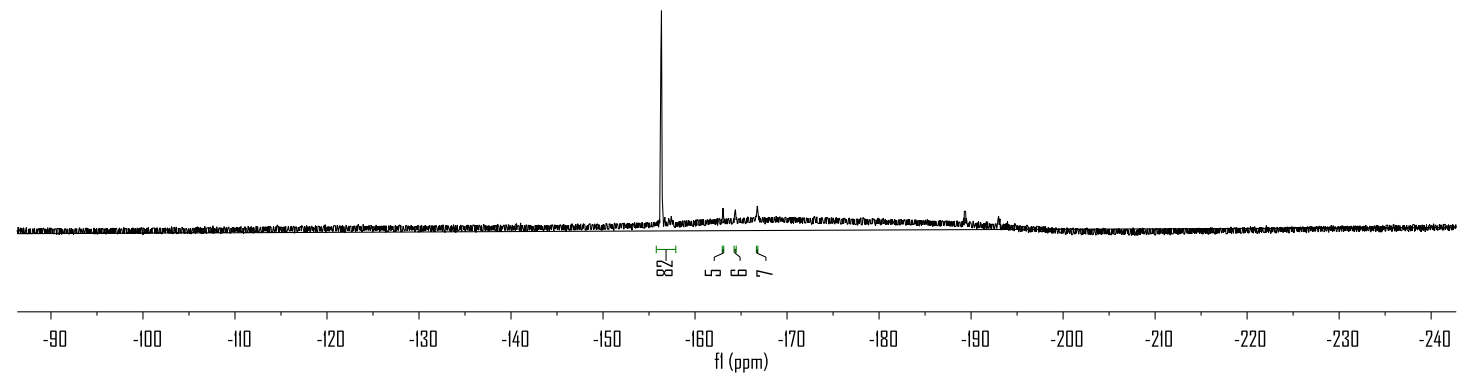

The stereoisomers are at $\delta-156.34(\mathrm{dd}, J=22.9,10.8 \mathrm{~Hz}),-163.05(\mathrm{~s}),-164.39(\mathrm{~s})$, $-166.76(\mathrm{~s})$ 
Crude 3gb, d.r. $=82: 18$<smiles>Cc1ccc(C(NS(=O)CC(C)(C)C)C(C)(F)C(=O)c2ccccc2)cc1</smiles>

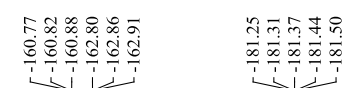

瓷

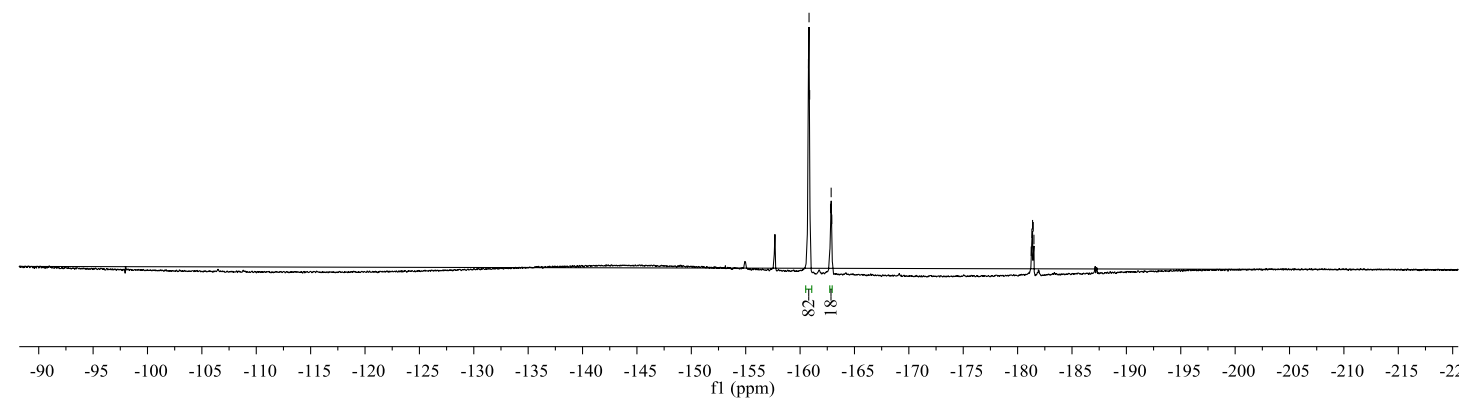

The stereoisomers are at $\delta-159.94--161.53(\mathrm{~m}),-162.71--163.05(\mathrm{~m})$. 
Crude 3ha, d.r. $=96: 4$

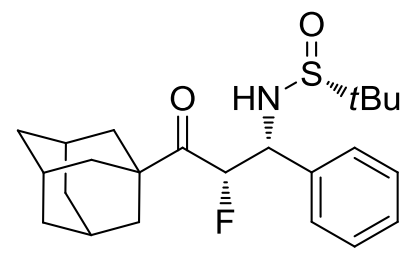

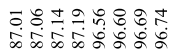

年

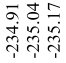

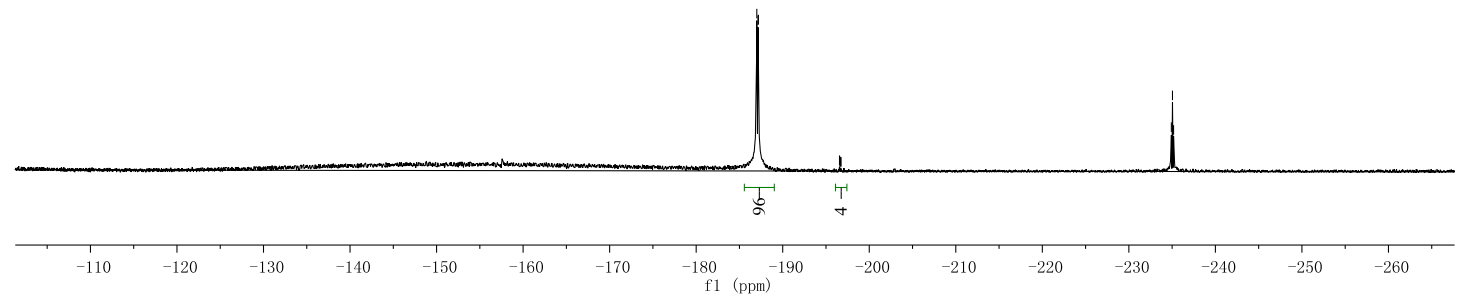

The stereoisomers are at $\delta-187.10(\mathrm{dd}, J=50.7,17.8 \mathrm{~Hz}),-196.65(\mathrm{dd}, J=49.4,16.9$ $\mathrm{Hz})$. 
Crude 3ib, d.r. $=96: 4$<smiles>Cc1ccc(C(NS(=O)C(C)(C)C)[C@H](F)C(=O)c2ccccc2C)cc1</smiles>

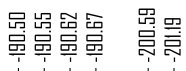

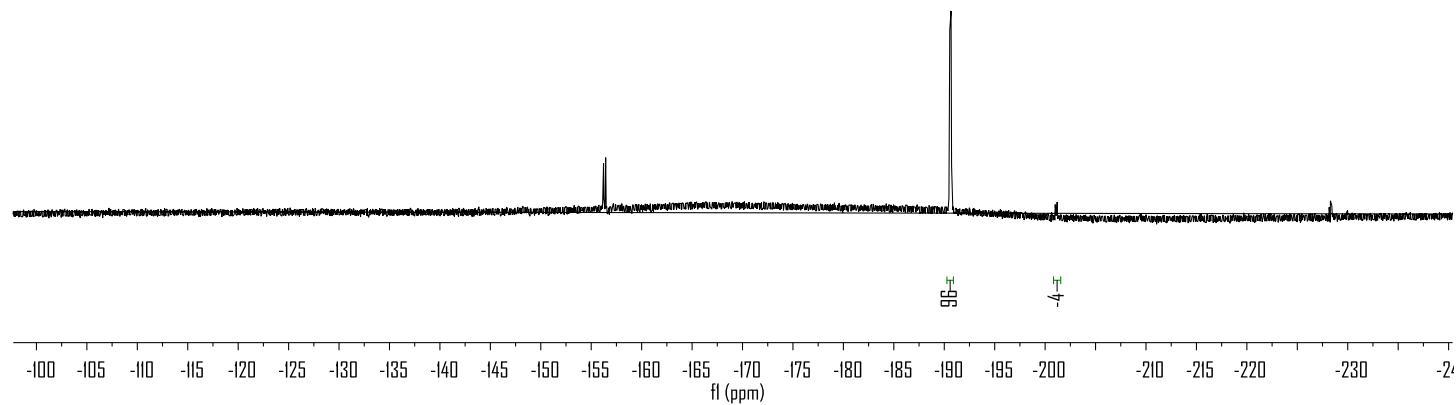

The stereoisomers are at $\delta-190.59(\mathrm{dd}, J=47.5,18.6 \mathrm{~Hz}),-201.19(\mathrm{~s})$. 
Crude 3jb, d.r. $=90: 8: 2$<smiles>CCCS(=O)N[C@H](c1ccc(C)cc1)[C@H](F)C(=O)c1ccccc1OC</smiles>

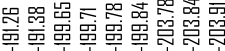

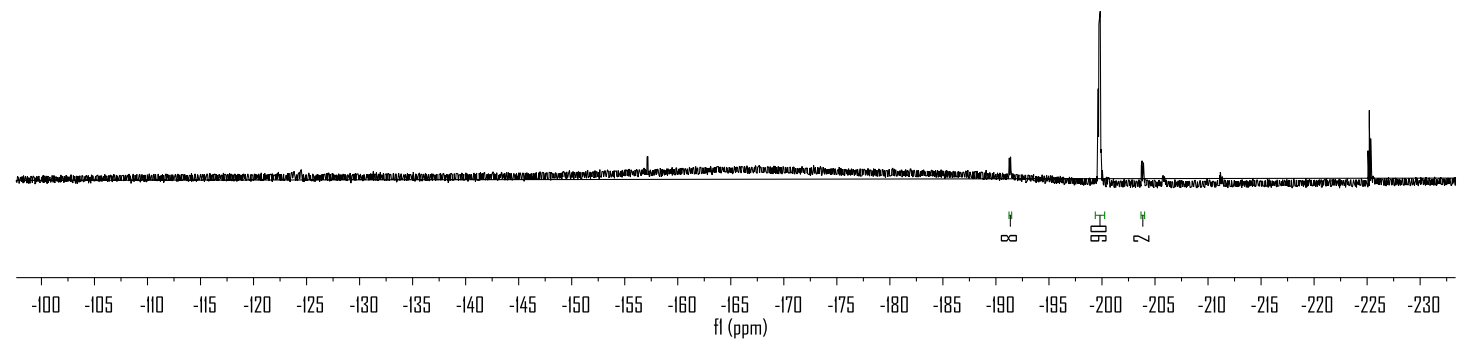

The stereoisomers are at $\delta-191.32(\mathrm{~d}, J=46.7 \mathrm{~Hz}),-199.74(\mathrm{dd}, J=47.7,22.4 \mathrm{~Hz})$, $-203.45--204.18(m)$. 
Crude 3ab, d.r. $=93: 7$<smiles>Cc1ccc(C(NS(=O)C(C)(C)C)C2(F)CCc3ccccc3C2=O)cc1</smiles>

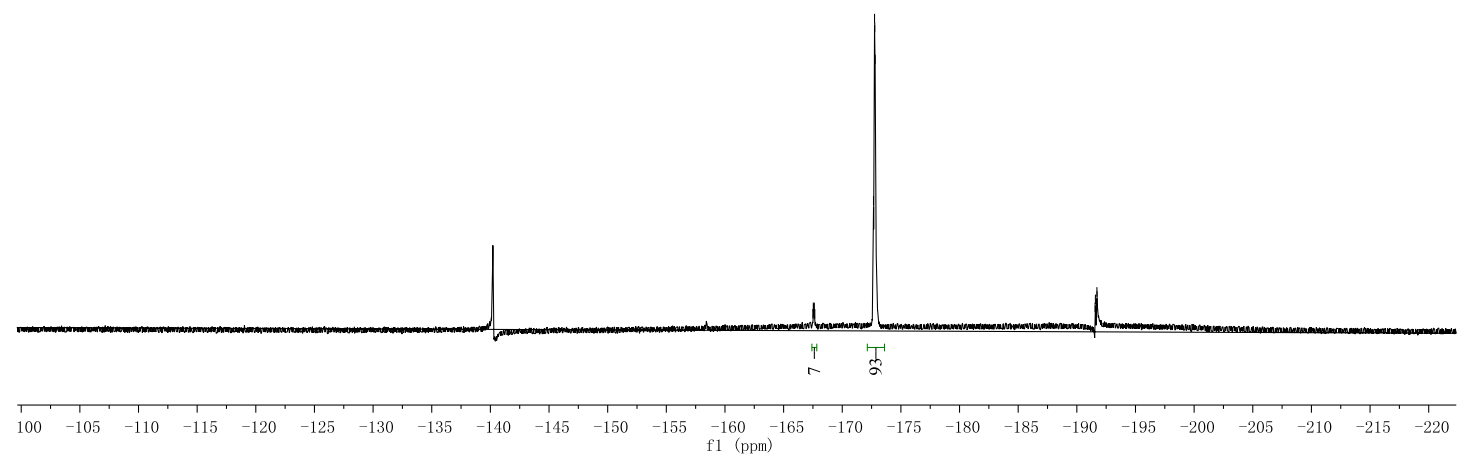

The stereoisomers are at $\delta-167.41--167.83(\mathrm{~m}),-172.75(\mathrm{dd}, J=29.4,22.2 \mathrm{~Hz})$. 
Crude 3ac, d.r. $=96: 4$<smiles>COc1ccccc1[C@H](NS(=O)C(C)(C)C)[C@H]1CCc2ccccc2C1=O</smiles>

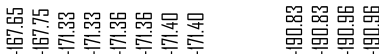

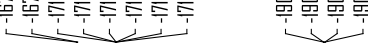

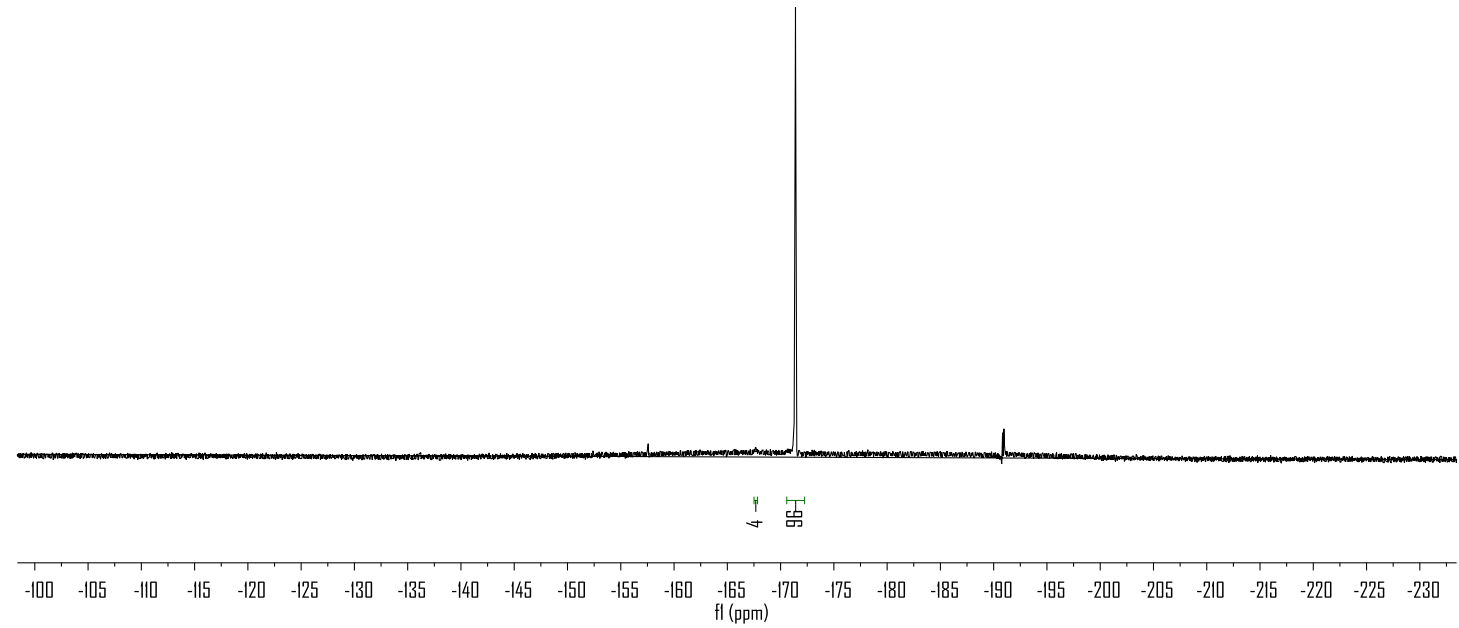

The stereoisomers are at $\delta-167.70(\mathrm{~d}, J=36.8 \mathrm{~Hz}),-170.58--172.26(\mathrm{~m})$. 
Crude 3ad, d.r. $=89: 7: 4$
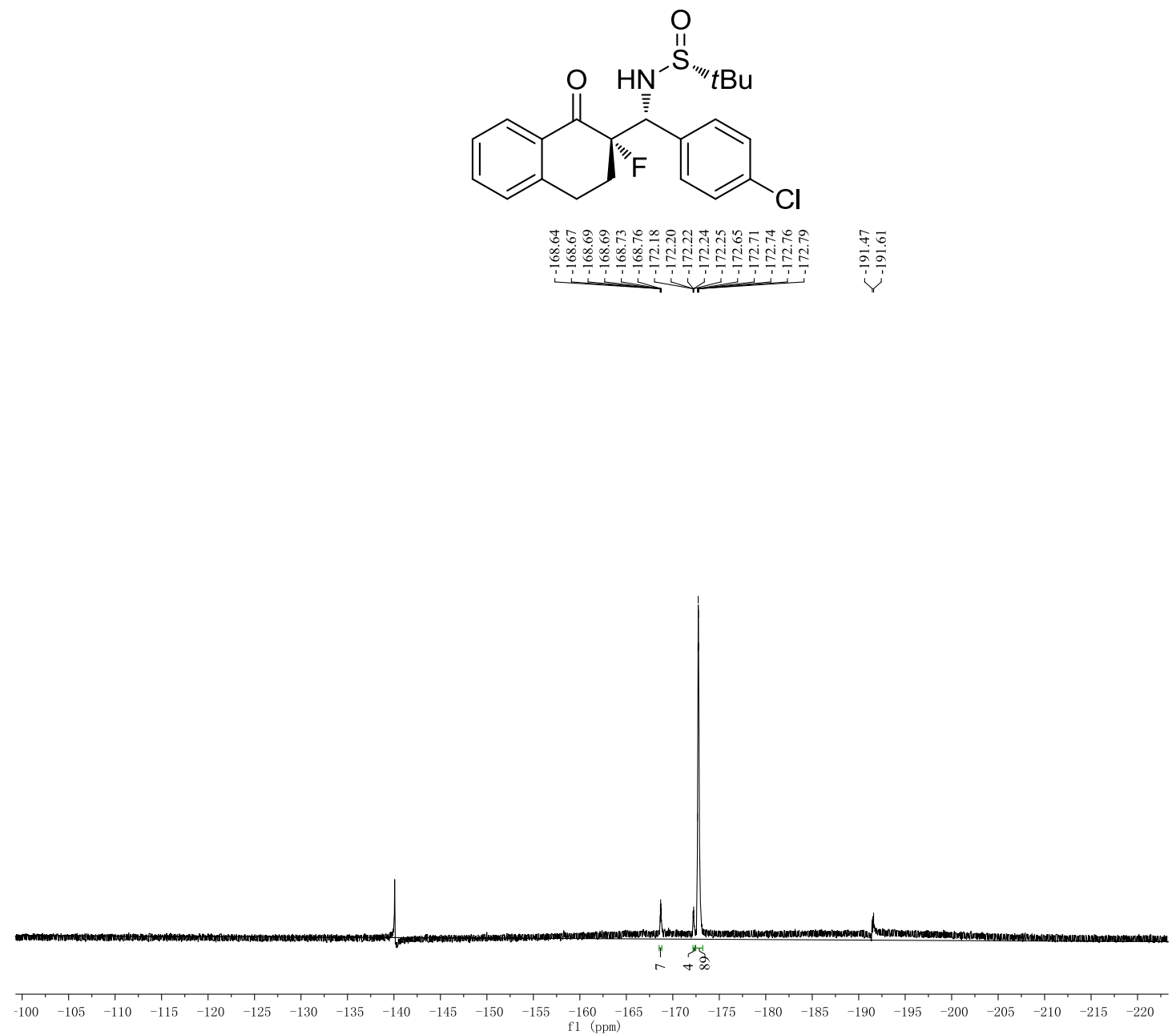

The stereoisomers are at $\delta-168.53--168.82(\mathrm{~m}),-172.17--172.38(\mathrm{~m}),-172.48-$ $-173.22(\mathrm{~m})$. 
Crude 3ae, d.r. $=90: 10$<smiles>CC(C)(C)S(=O)N[C@H](c1ccco1)C1(F)CCc2ccccc2C1=O</smiles>

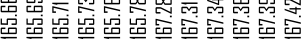

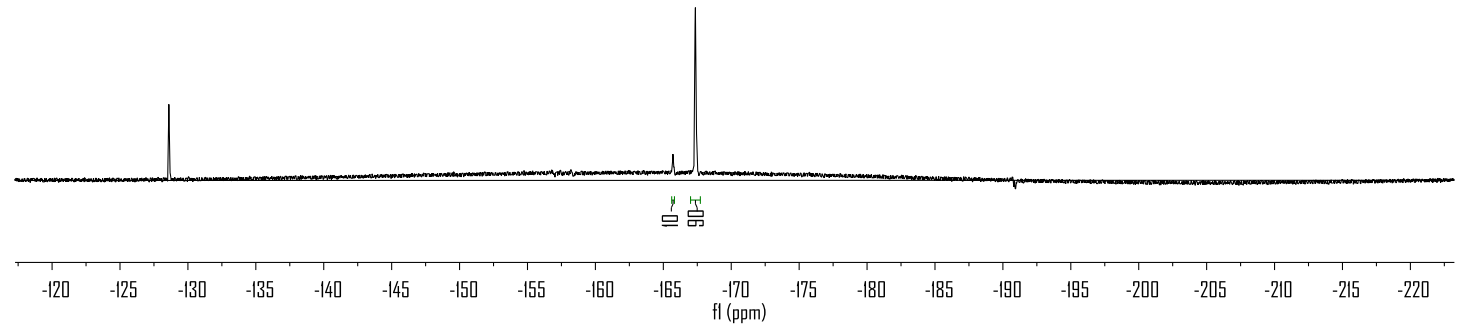

The stereoisomers are at $\delta-165.72(\mathrm{td}, J=20.5,10.4 \mathrm{~Hz}),-167.35(\mathrm{td}, J=20.5,10.6$ $\mathrm{Hz})$. 
Crude 3af, d.r. > 99:1<smiles>CC(C)(C)[S@](=O)N[C@H](/C=C/c1ccccc1)C1(F)CCc2ccccc2C1=O</smiles>

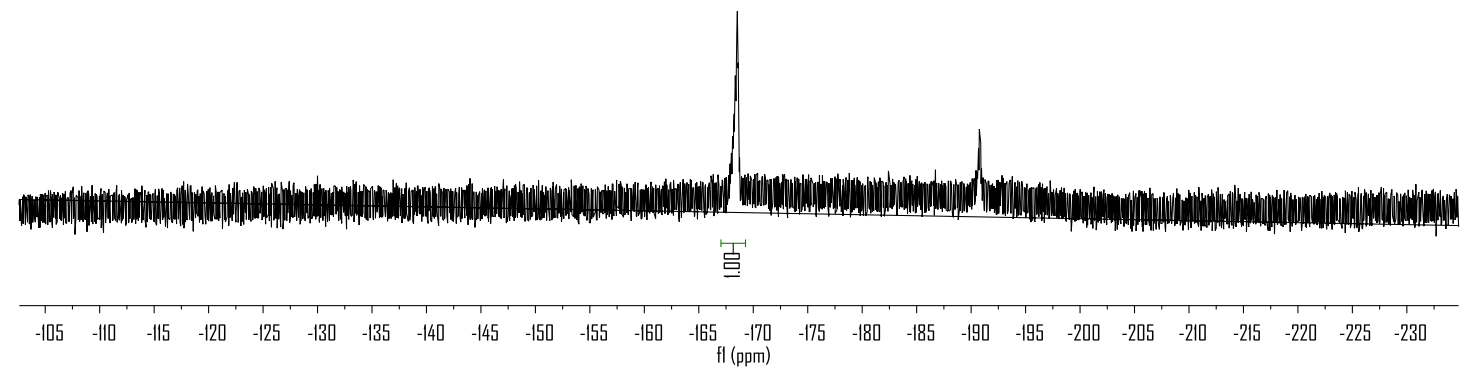

The stereoisomer is at $\delta-168.54$ (br). 
Crude 3bg, d.r. $=$ 90:10
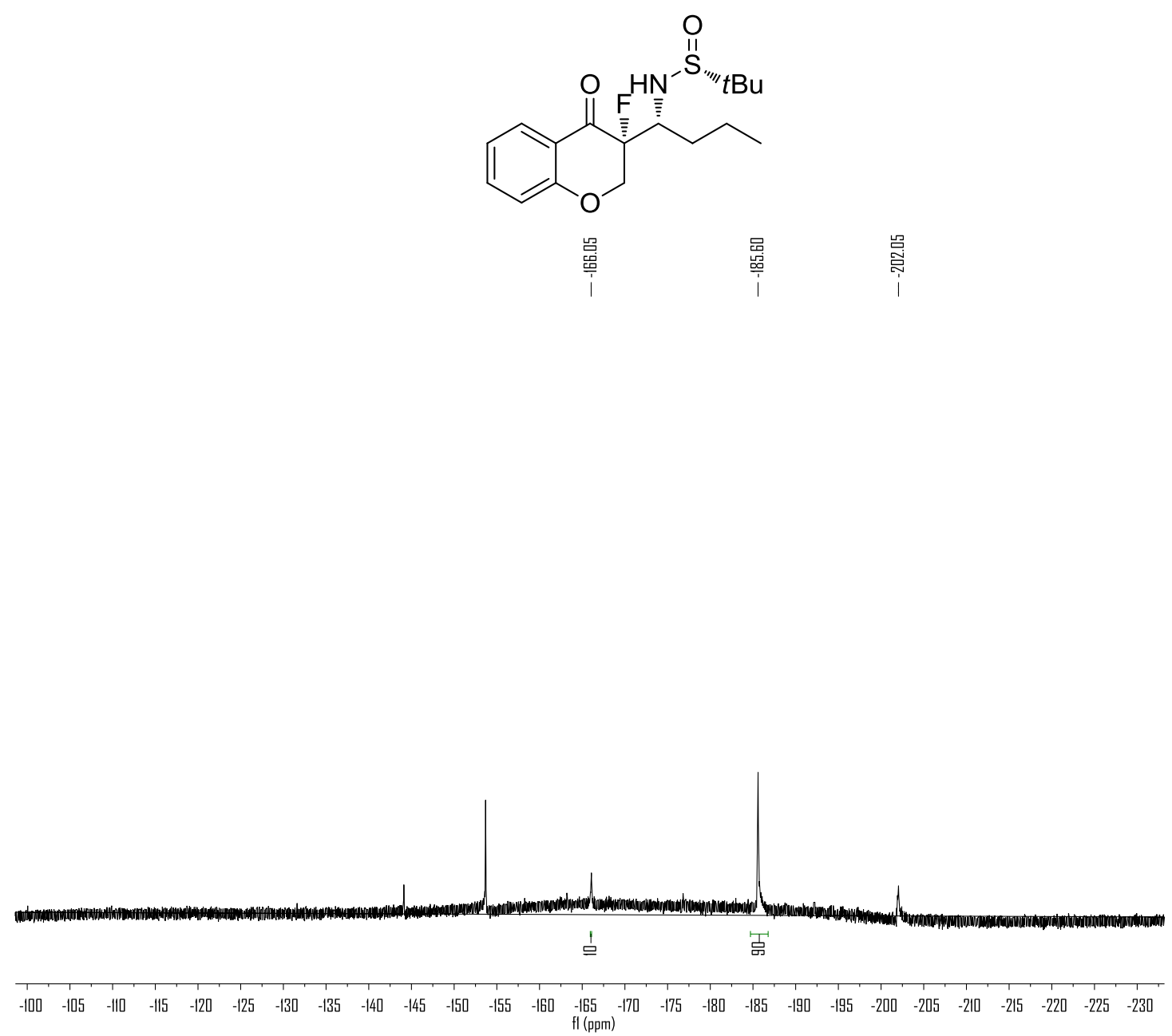

The stereoisomers are at $\delta-166.05$ (br), -185.60 (br). 
Crude 3bh, d.r. $=86: 8: 6$

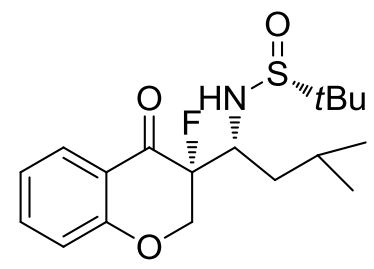

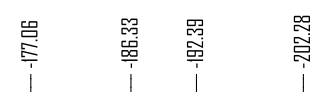

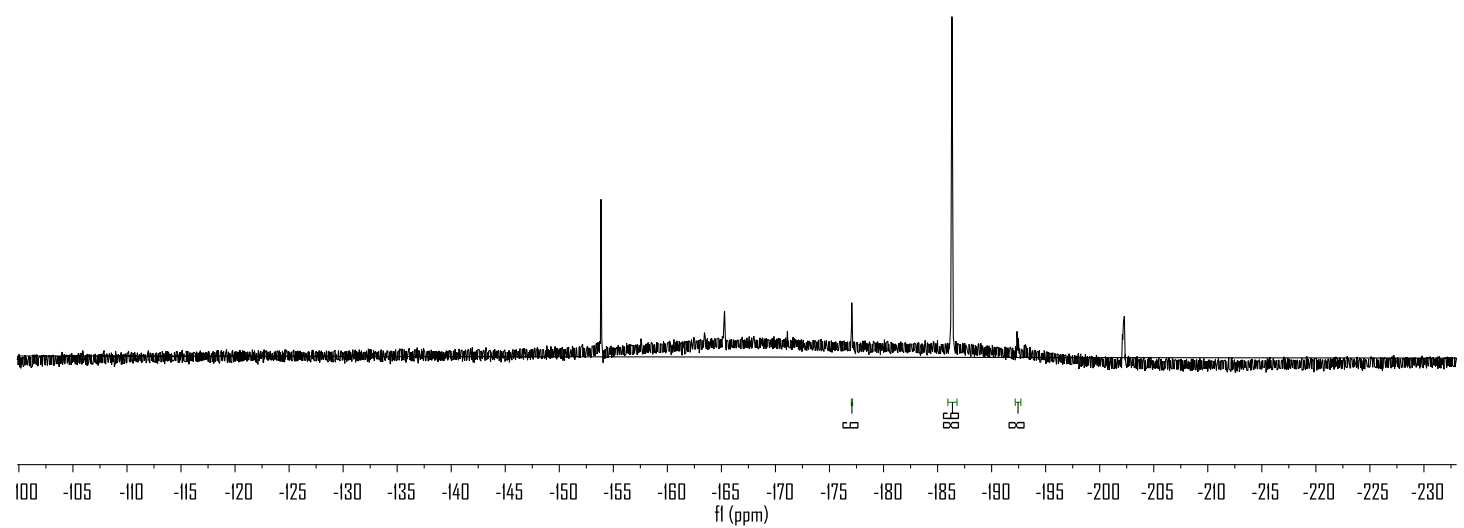

The stereoisomers are at $\delta-177.06$ (br), -186.33 (br), -192.39 (br). 
Crude 3db, d.r. $=96: 4$
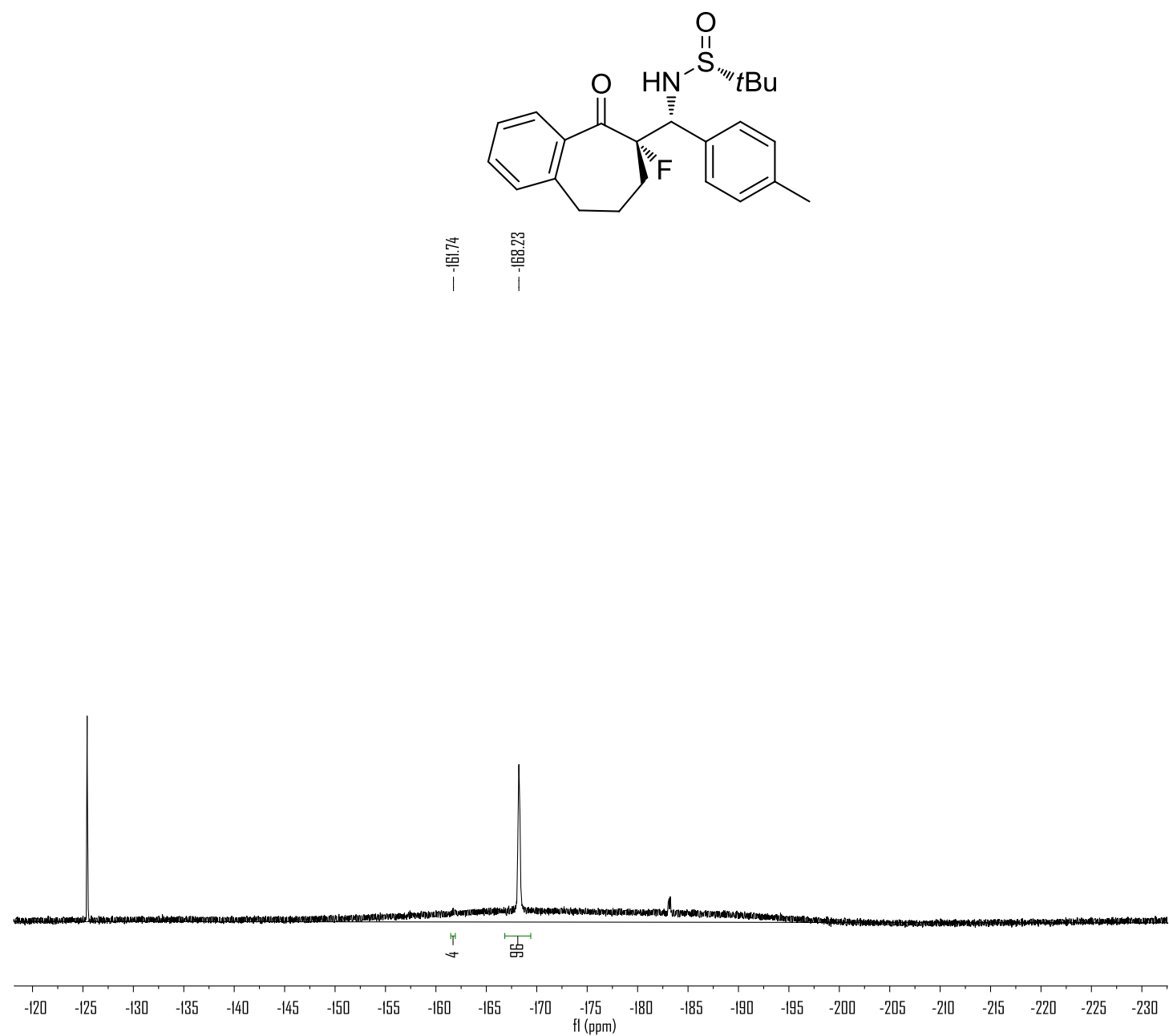

The stereoisomers are at $\delta-161.74$ (br), -168.23 (br). 
Crude 3gd, d.r. = 91:9
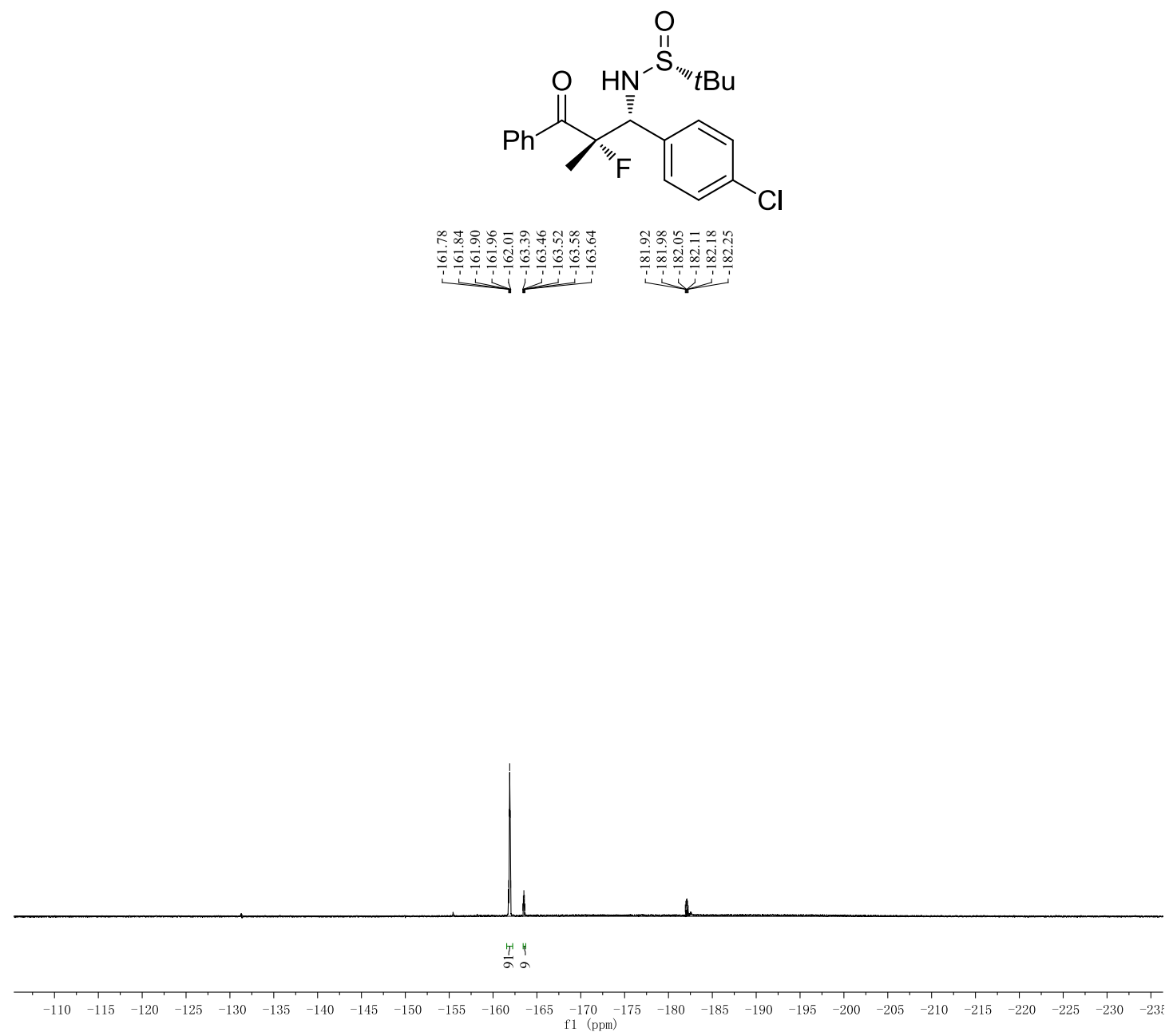

The stereoisomers are at $\delta-161.90(\mathrm{p}, J=21.7 \mathrm{~Hz}),-163.52(\mathrm{p}, J=23.5 \mathrm{~Hz})$. 
Crude 3hb, d.r.>99:1

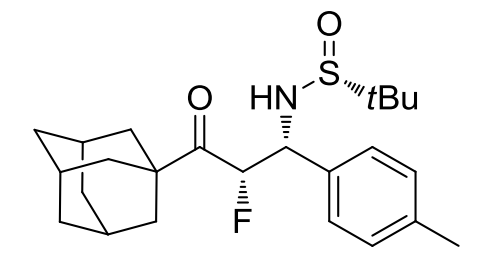

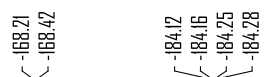

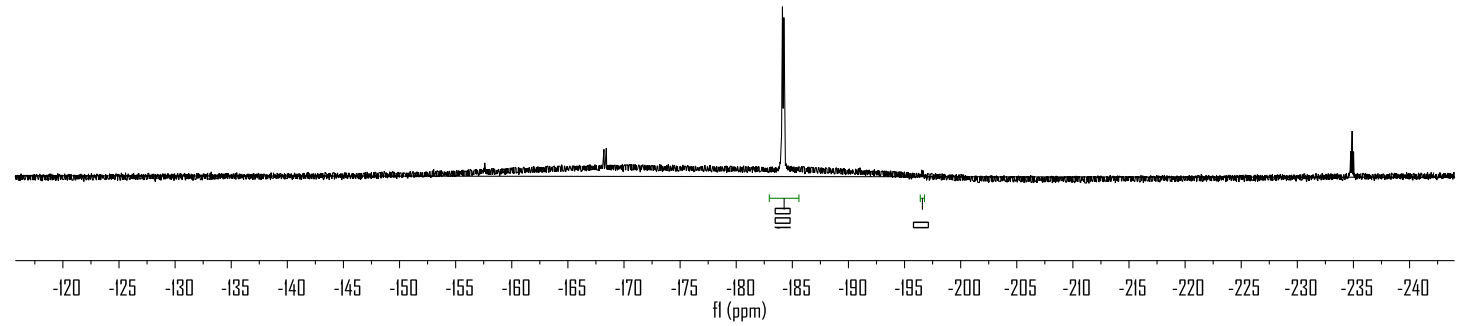

The stereoisomers are at $\delta-184.20(\mathrm{dd}, J=47.7,14.3 \mathrm{~Hz}),-196.40--196.78(\mathrm{~m})$ 
Crude 3hi, d.r. $=99: 1$

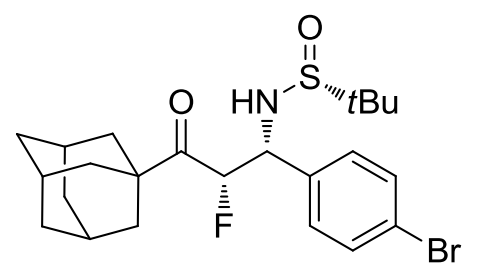

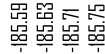

率策器

i i

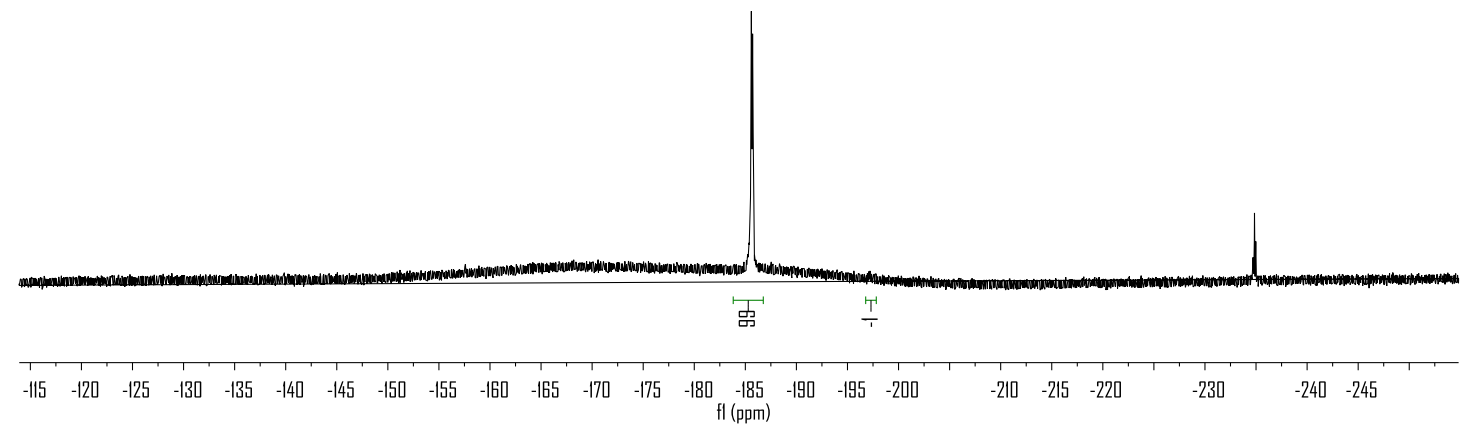

The stereoisomers are at $\delta-185.67(\mathrm{dd}, J=46.6,15.5 \mathrm{~Hz}),-196.78--197.82(\mathrm{~m})$. 
Crude 3hj, d.r. =99:1

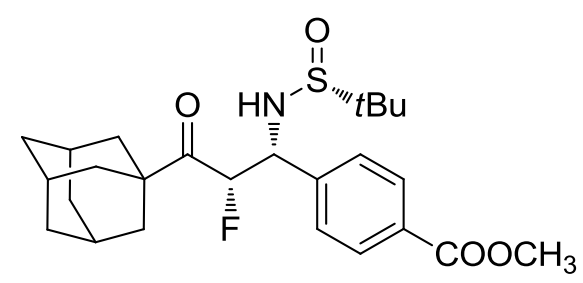

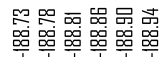

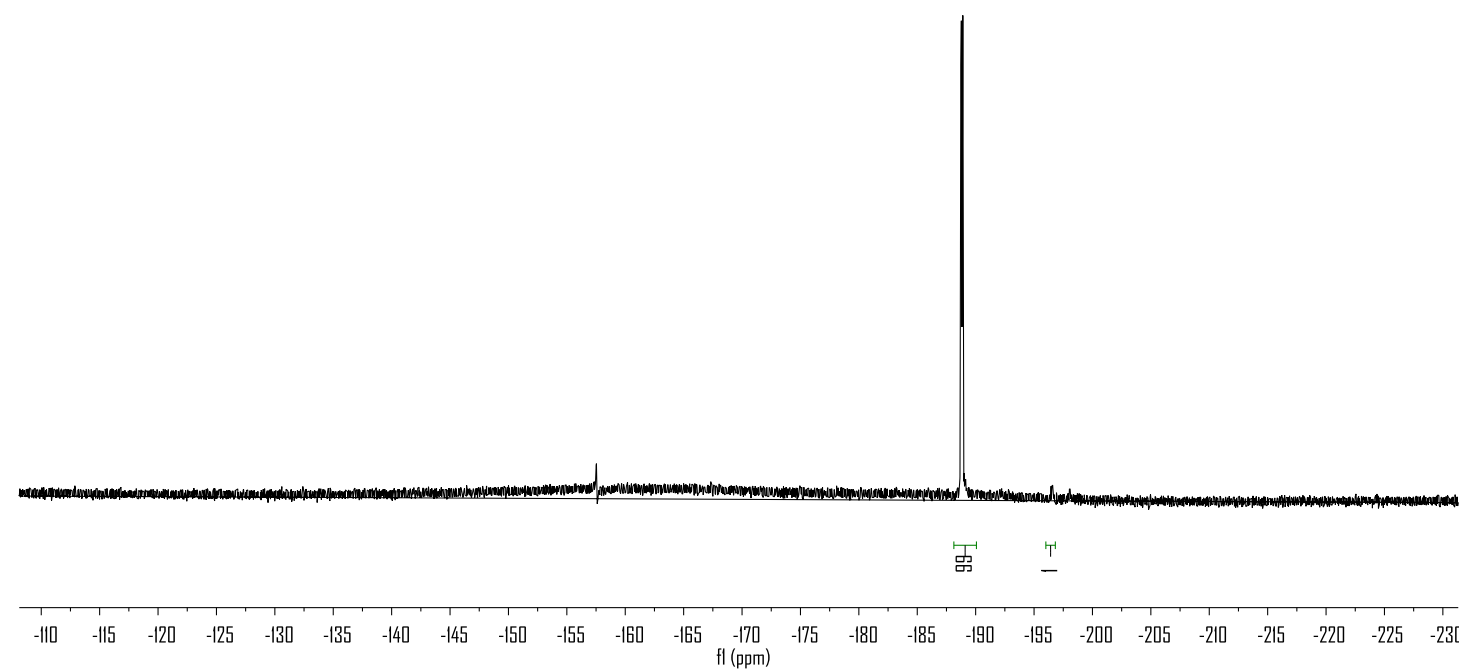

The stereoisomers are at $\delta-188.84(\mathrm{dt}, J=46.4,15.2 \mathrm{~Hz}),-196.01--196.82(\mathrm{~m})$. 
Crude 3hk, d.r. $=99: 1$

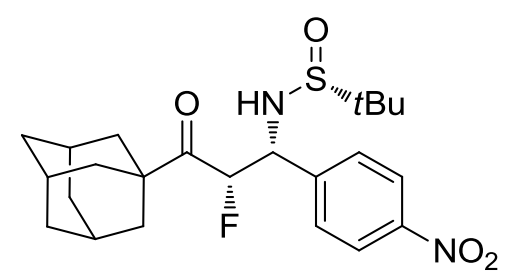

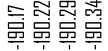

ij

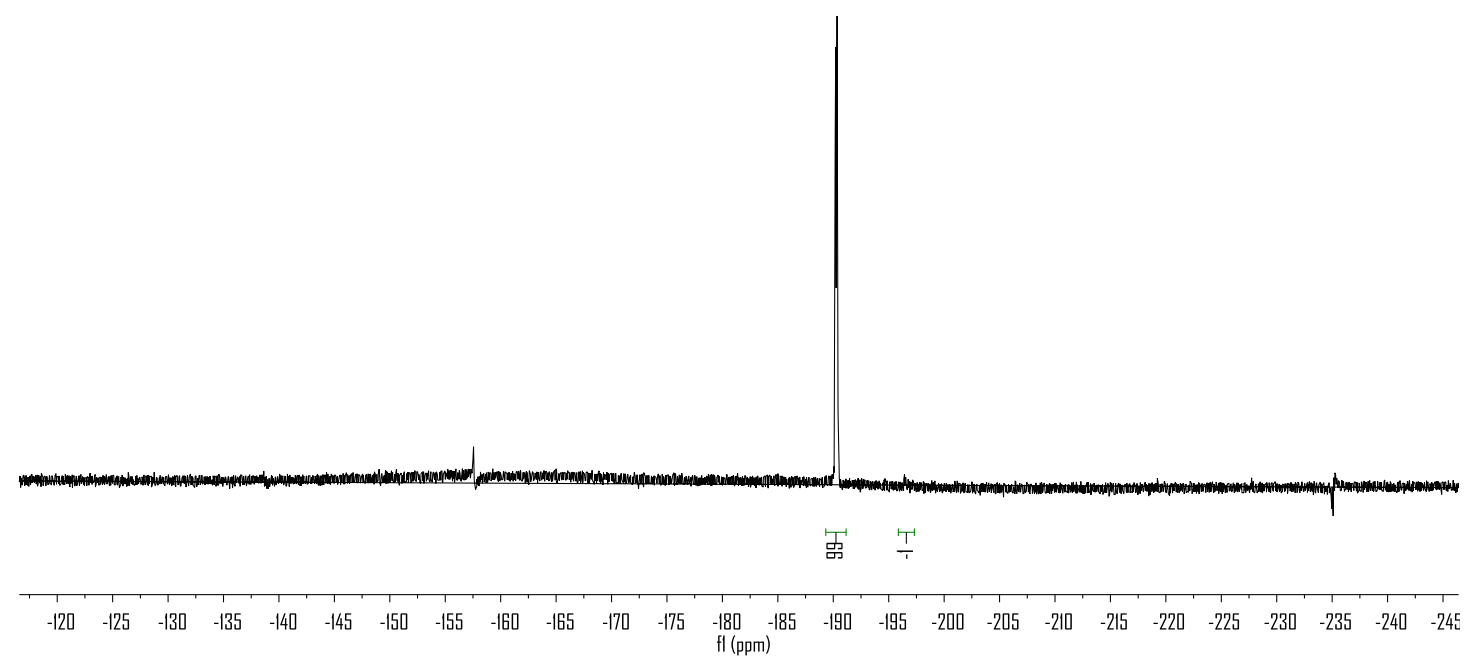

The stereoisomers are at $\delta-190.26(\mathrm{dd}, J=46.2,18.2 \mathrm{~Hz}),-195.87--197.31(\mathrm{~m})$. 
Crude 3hl, d.r. $=99: 1$<smiles>CC(C)(C)S(=O)N[C@H](c1ccc(C#N)cc1)[C@@H](F)C(=O)C12CC3CC(CC(C3)C1)C2</smiles>

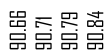

i

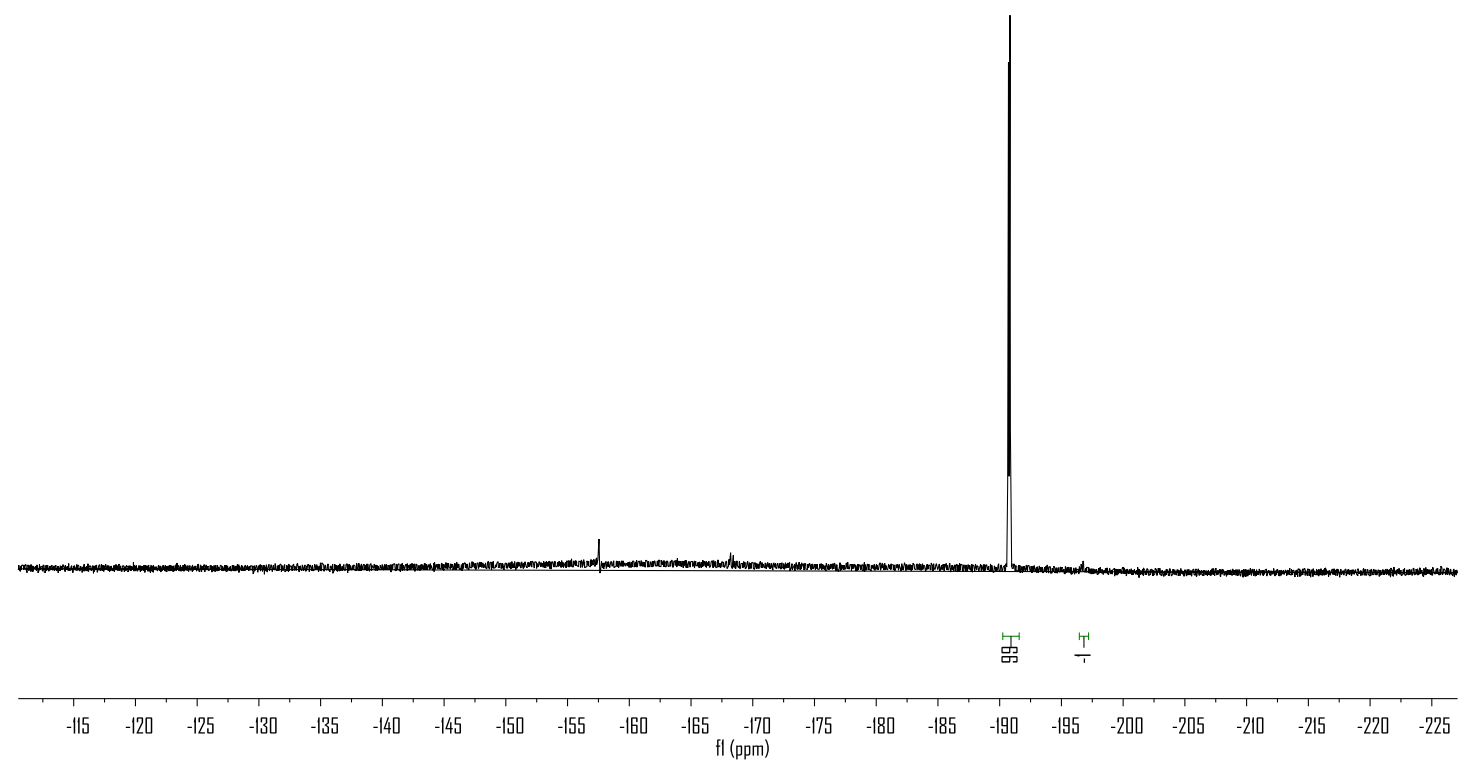

The stereoisomers are at $\delta-190.75(\mathrm{dd}, J=46.6,18.4 \mathrm{~Hz}),-196.45--197.20(\mathrm{~m})$. 
Crude 3ji, d.r. $=88: 7: 4: 1$<smiles>COc1ccccc1C(=O)[C@H](F)[C@H](NS(=O)C(C)(C)C)c1ccc(Br)cc1</smiles>

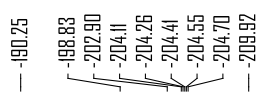

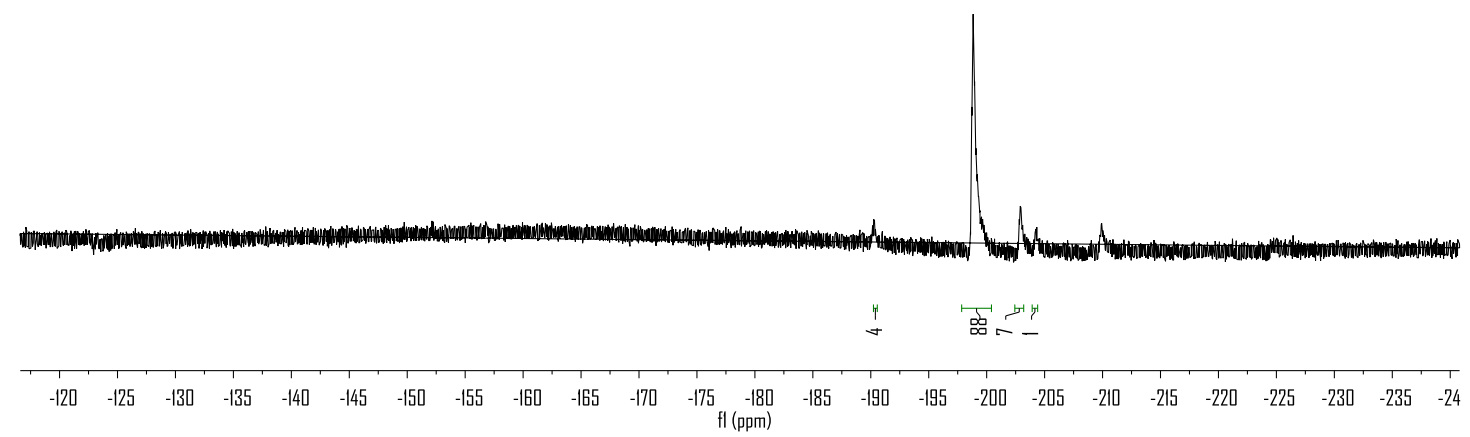

The stereoisomers are at $\delta-190.25$ (br), -198.83 (br), -202.90 (br), -203.93 - -204.40 (m). 
Crude 3na, d.r. $=91: 9$<smiles>CC(C)(C)C(=O)[C@H](F)[C@H](NS(=O)C(C)(C)C)c1ccccc1</smiles>

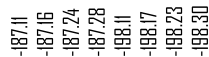

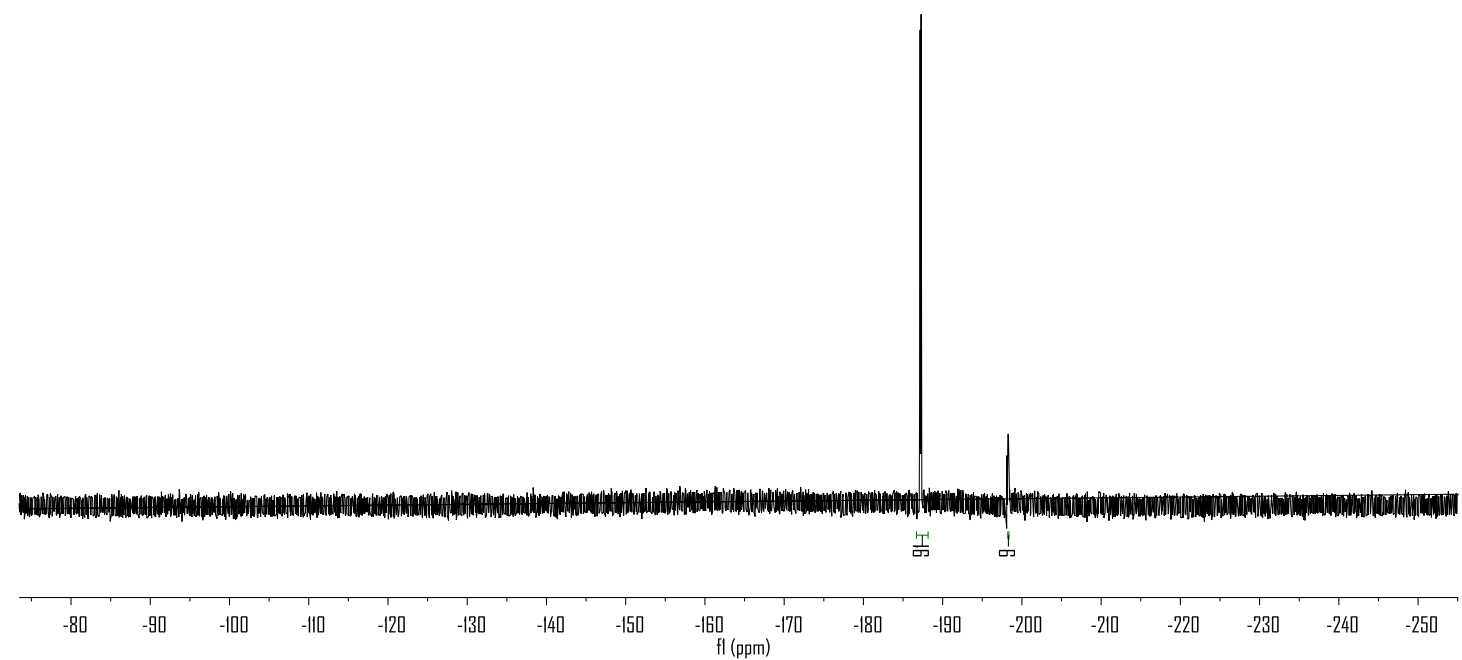

The stereoisomers are at $\delta-187.20(\mathrm{dd}, J=47.4,16.6 \mathrm{~Hz}),-198.20(\mathrm{dd}, J=48.6,24.4$ $\mathrm{Hz})$. 


\section{NMR Spectra for All New Compounds}

${ }^{1} \mathrm{H}$ NMR (400 MHz, $\mathrm{CDCl}_{3}$ ) spectrum of 3aa<smiles>CC(C)(C)S(=O)N[C@H](c1ccccc1)C1(F)CCc2ccccc2C1=O</smiles>

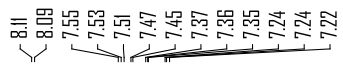

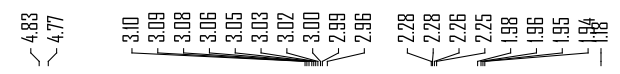

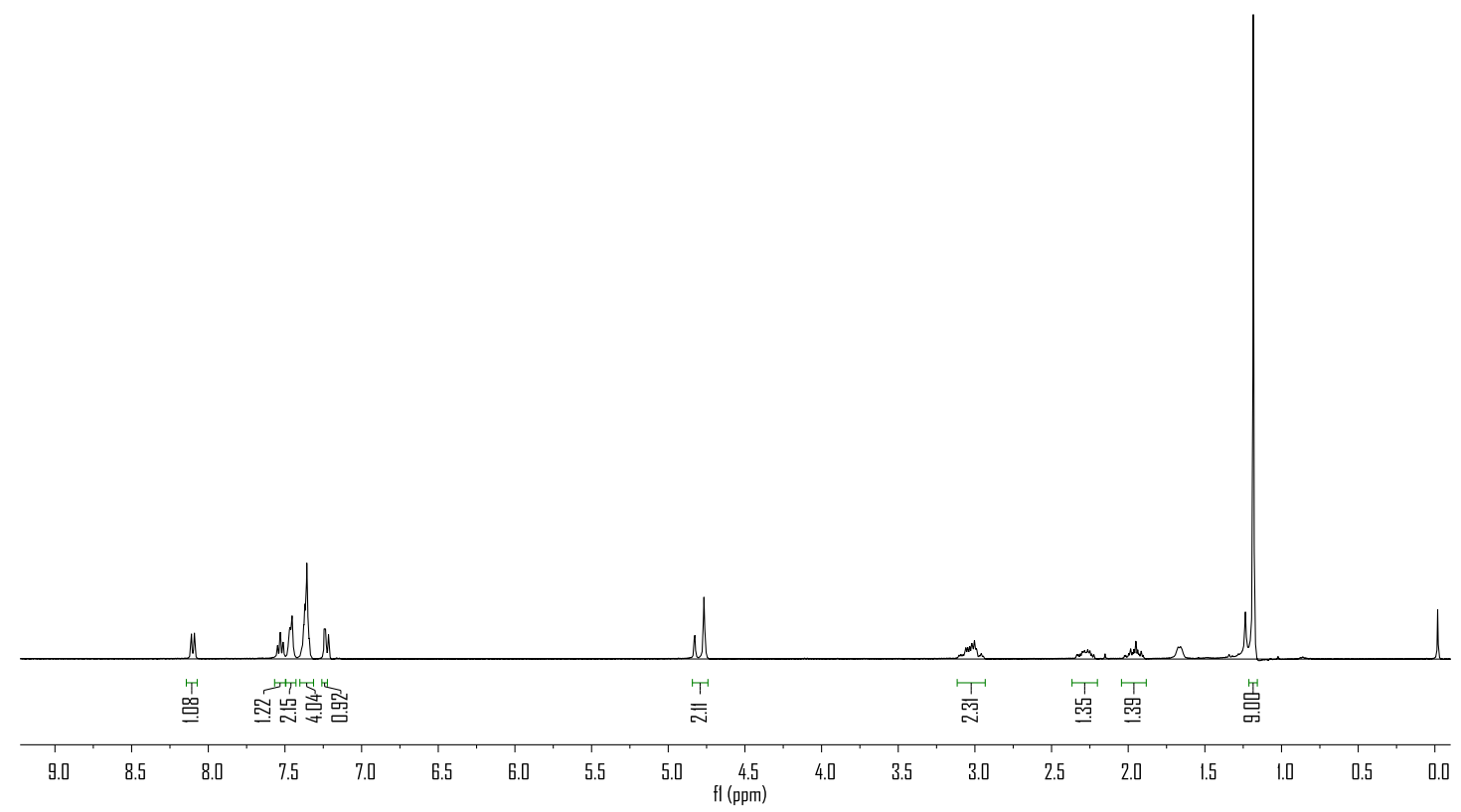


${ }^{19} \mathrm{~F}$ NMR (376 MHz, $\mathrm{CDCl}_{3}$ ) spectrum of 3aa<smiles>CC(C)(C)S(=O)N[C@H](c1ccccc1)C1(F)CCc2ccccc2C1=O</smiles>

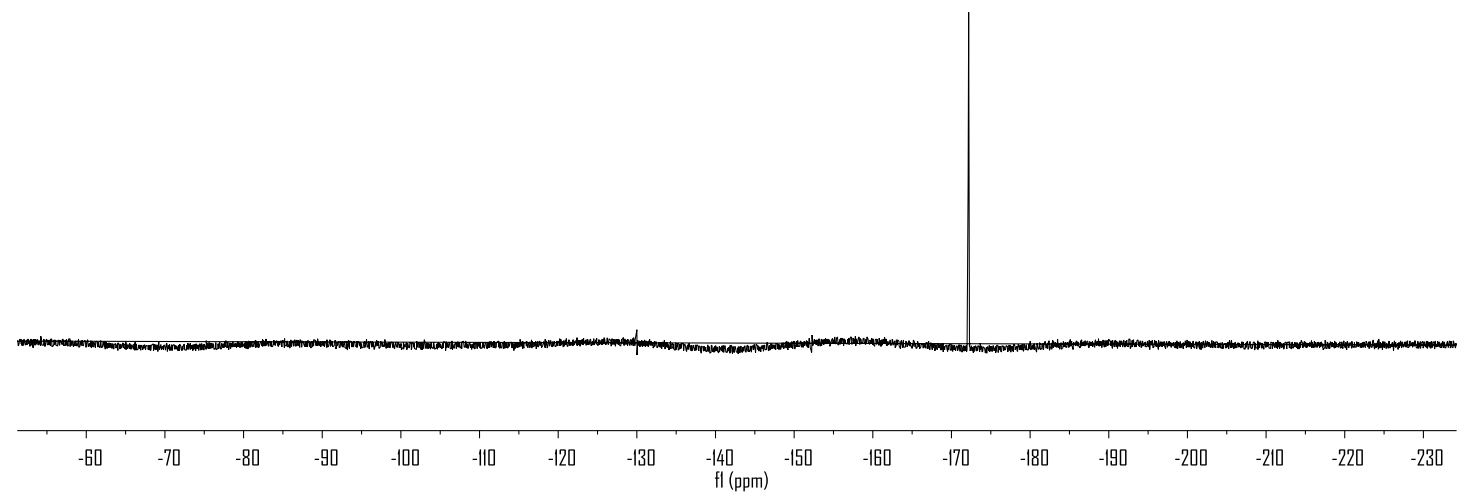


${ }^{13} \mathrm{C}$ NMR (101 MHz, $\mathrm{CDCl}_{3}$ ) spectrum of 3aa<smiles>[14CH3][S@](=O)N[C@H](c1ccccc1)[C-]1CCc2ccccc2C1=O</smiles>

罍咆

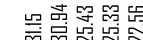

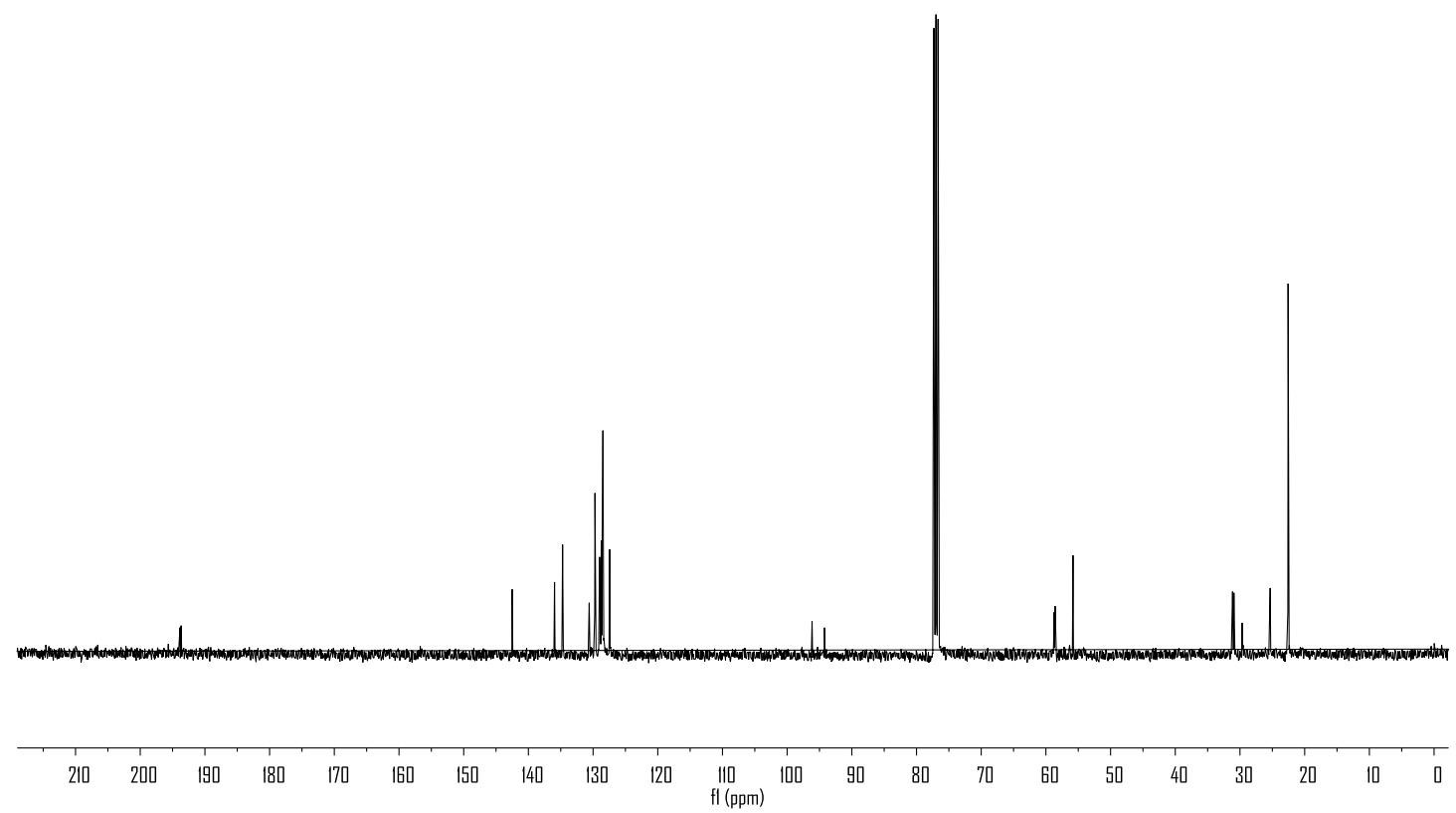


${ }^{1} \mathrm{H}$ NMR (400 MHz, $\mathrm{CDCl}_{3}$ ) spectrum of 3aa'<smiles>[14CH3]S(=O)N[C@H](c1ccccc1)[C@H]1CCc2ccccc2C1=O</smiles>

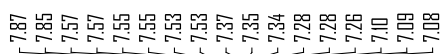

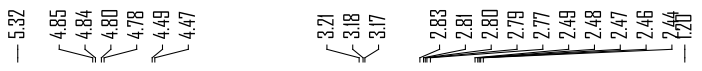

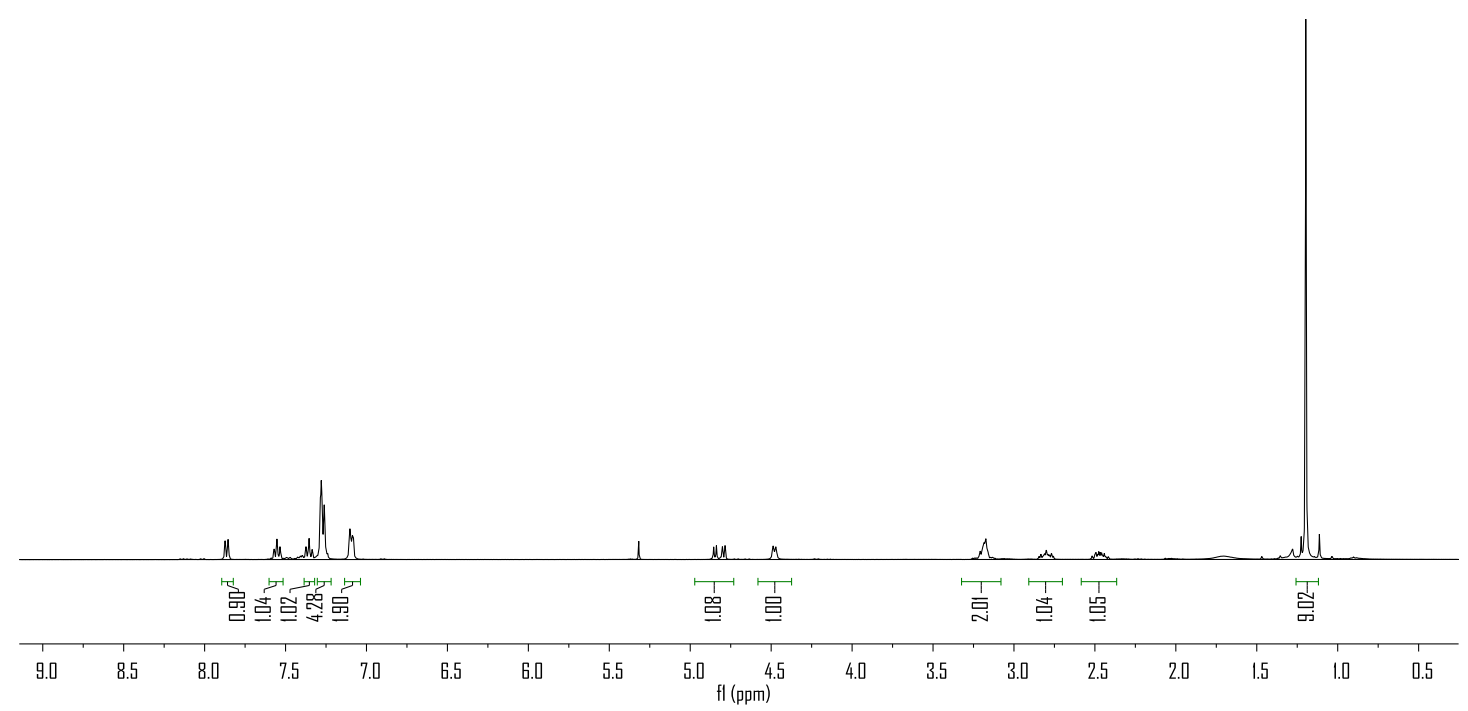


${ }^{19} \mathrm{~F}$ NMR $\left(376 \mathrm{MHz}, \mathrm{CDCl}_{3}\right.$ ) spectrum of 3aa'<smiles>[14CH3]S(=O)N[C@H](c1ccccc1)[C@@H]1CCc2ccccc2C1=O</smiles>

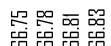

i

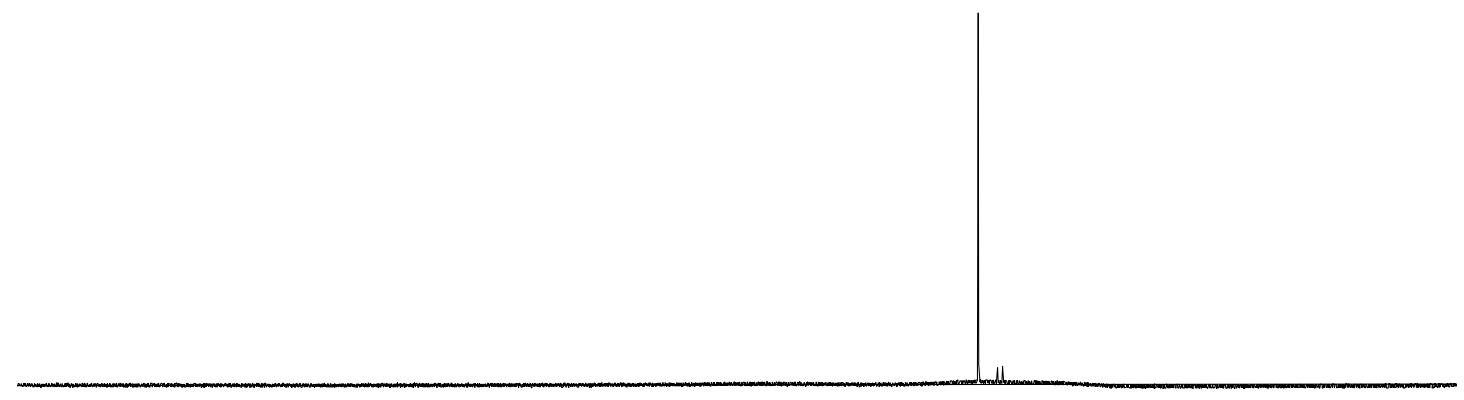

$\begin{array}{lllllllllllllllllllllllllllllllllllllll}90 & 80 & 70 & 60 & 50 & 40 & 30 & 20 & 10 & 0 & -20 & -40 & -60 & -80 & -100 & -120 & -140 & -160 & -180 & -200 & -220 & -240 & -260 & -280\end{array}$ 
${ }^{13} \mathrm{C}$ NMR $\left(101 \mathrm{MHz}, \mathrm{CDCl}_{3}\right.$ ) spectrum of 3aa'

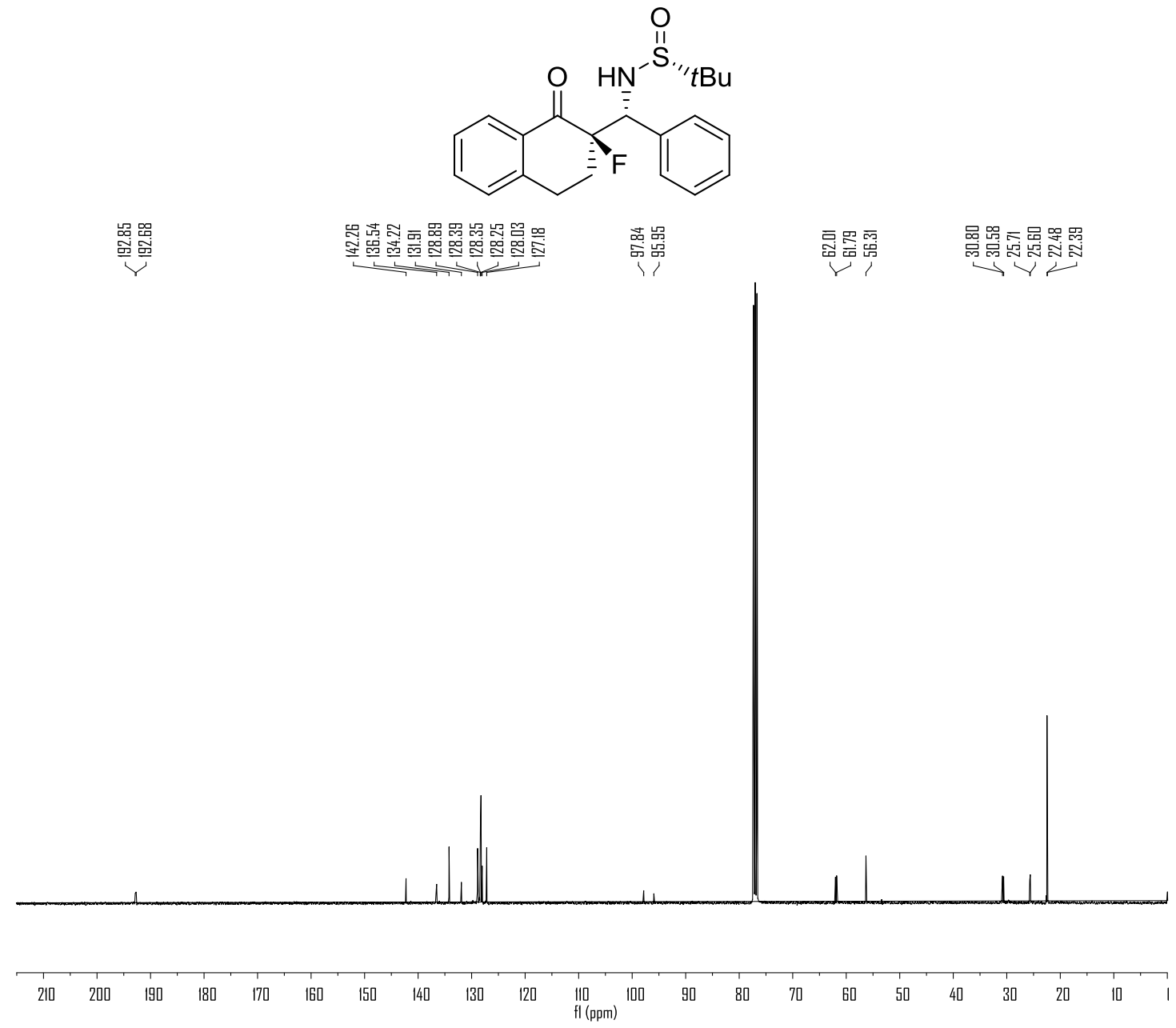


${ }^{1} \mathrm{H}$ NMR (400 MHz, $\mathrm{CDCl}_{3}$ ) spectrum of $\mathbf{3 b a}$<smiles>CC(C)(C)S(=O)N[C@H](c1ccccc1)C1(F)COc2ccccc2C1=O</smiles>

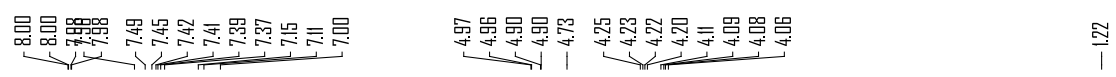

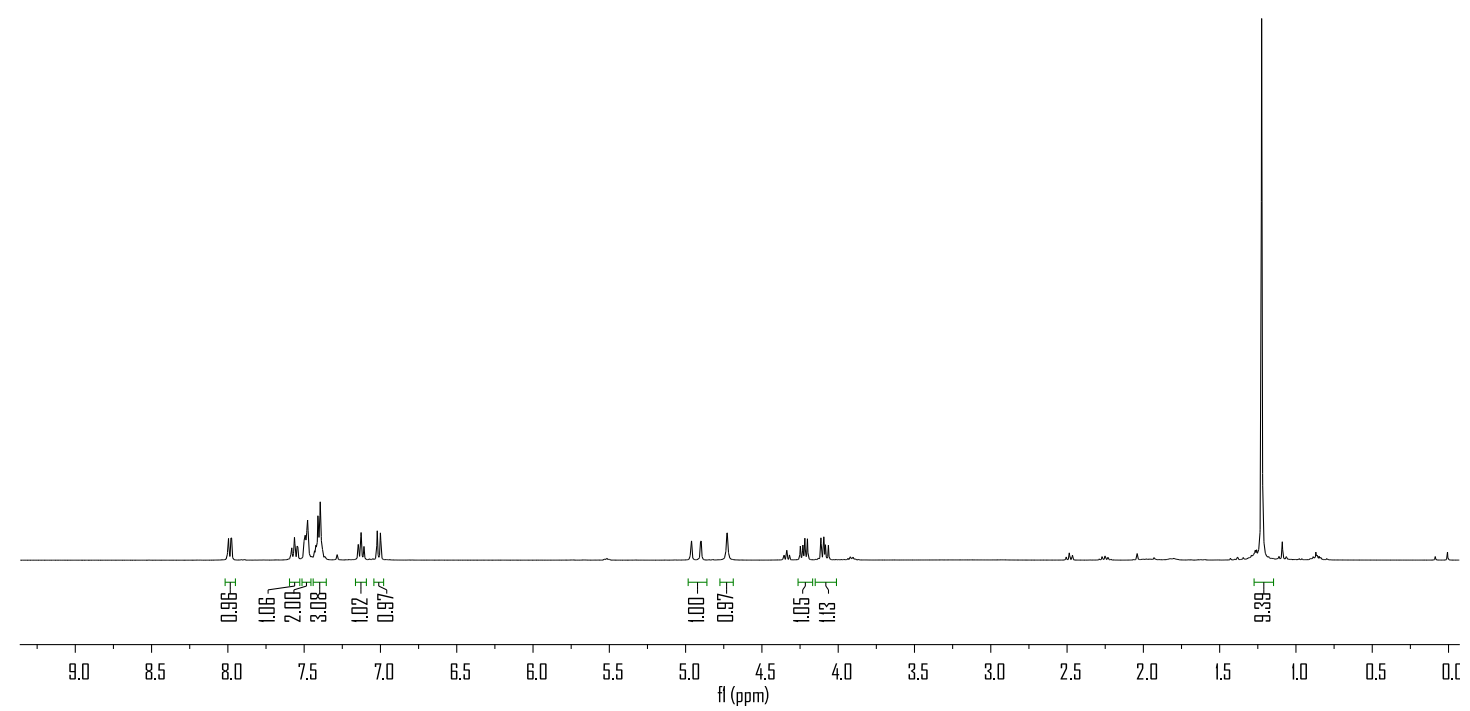


${ }^{19} \mathrm{~F}$ NMR $\left(376 \mathrm{MHz}, \mathrm{CDCl}_{3}\right)$ spectrum of $\mathbf{3 b a}$<smiles>CC(C)(C)S(=O)N[C@H](c1ccccc1)C1(F)COc2ccccc2C1=O</smiles>

表紫

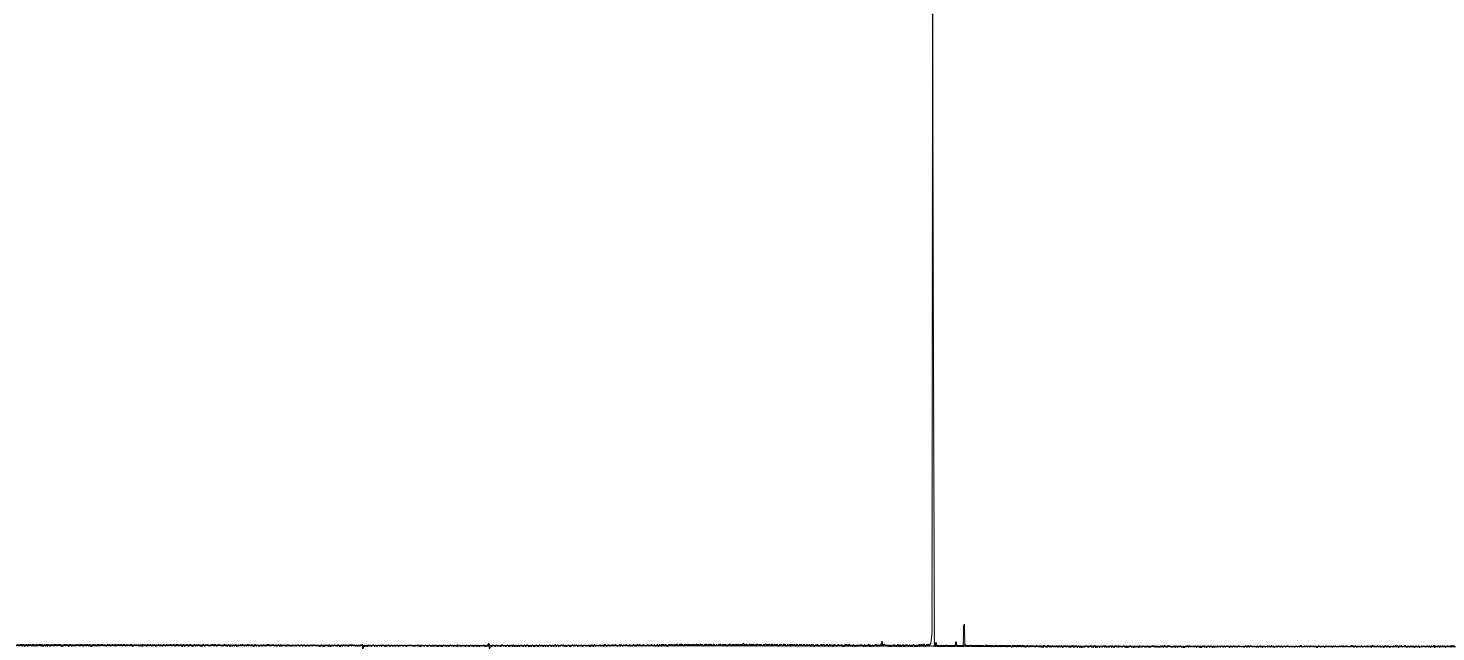

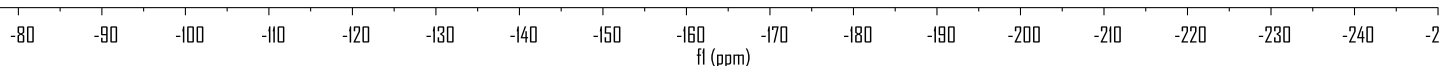


${ }^{13} \mathrm{C}$ NMR $\left(101 \mathrm{MHz}, \mathrm{CDCl}_{3}\right)$ spectrum of $\mathbf{3 b a}$

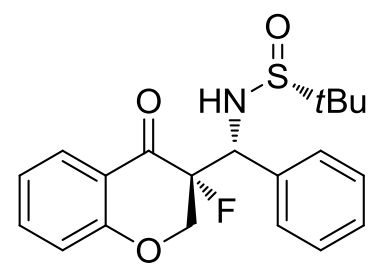

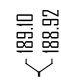

嘼

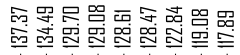

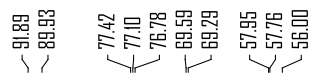

品

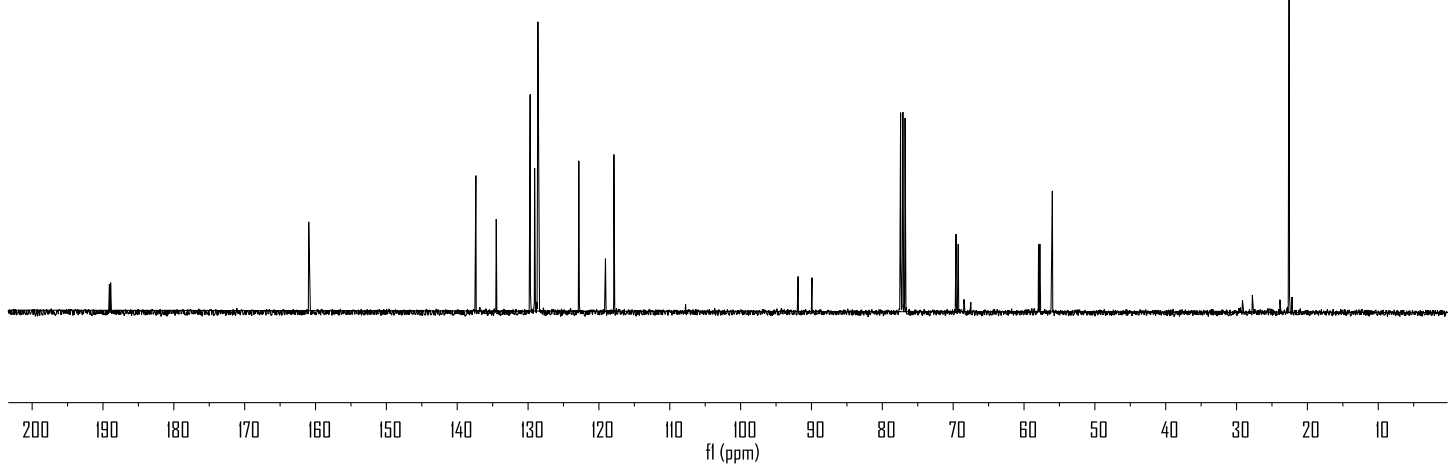

43 
${ }^{1} \mathrm{H}$ NMR (400 MHz, $\mathrm{CDCl}_{3}$ ) spectrum of $\mathbf{3 b a}$ '<smiles>O=C1c2ccccc2OCC1(NNS(=O)[18O])c1ccccc1</smiles>

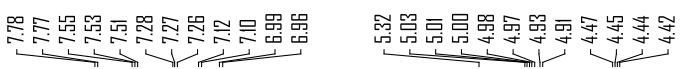

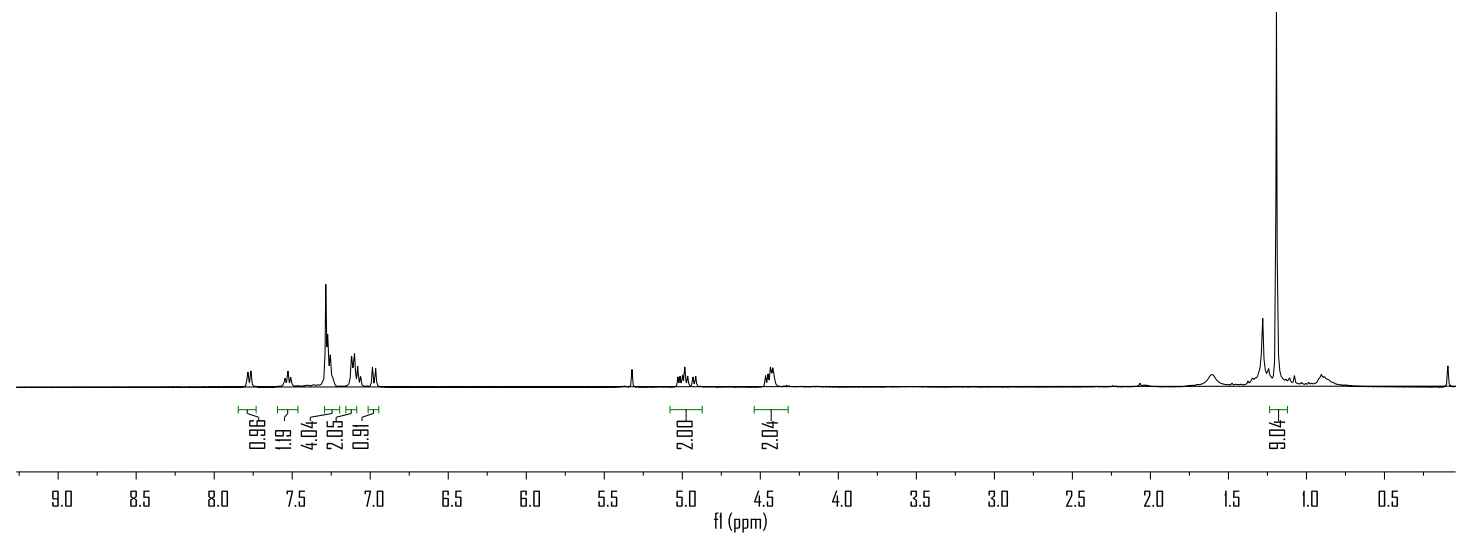


${ }^{19} \mathrm{~F}$ NMR $\left(376 \mathrm{MHz}, \mathrm{CDCl}_{3}\right)$ spectrum of $\mathbf{3 b a}$ '<smiles>CC(C)(C)S(=O)N[C@H](c1ccccc1)C1(F)COc2ccccc2C1=O</smiles> 
${ }^{13} \mathrm{C}$ NMR (101 MHz, $\mathrm{CDCl}_{3}$ ) spectrum of $3 \mathbf{b a a}$

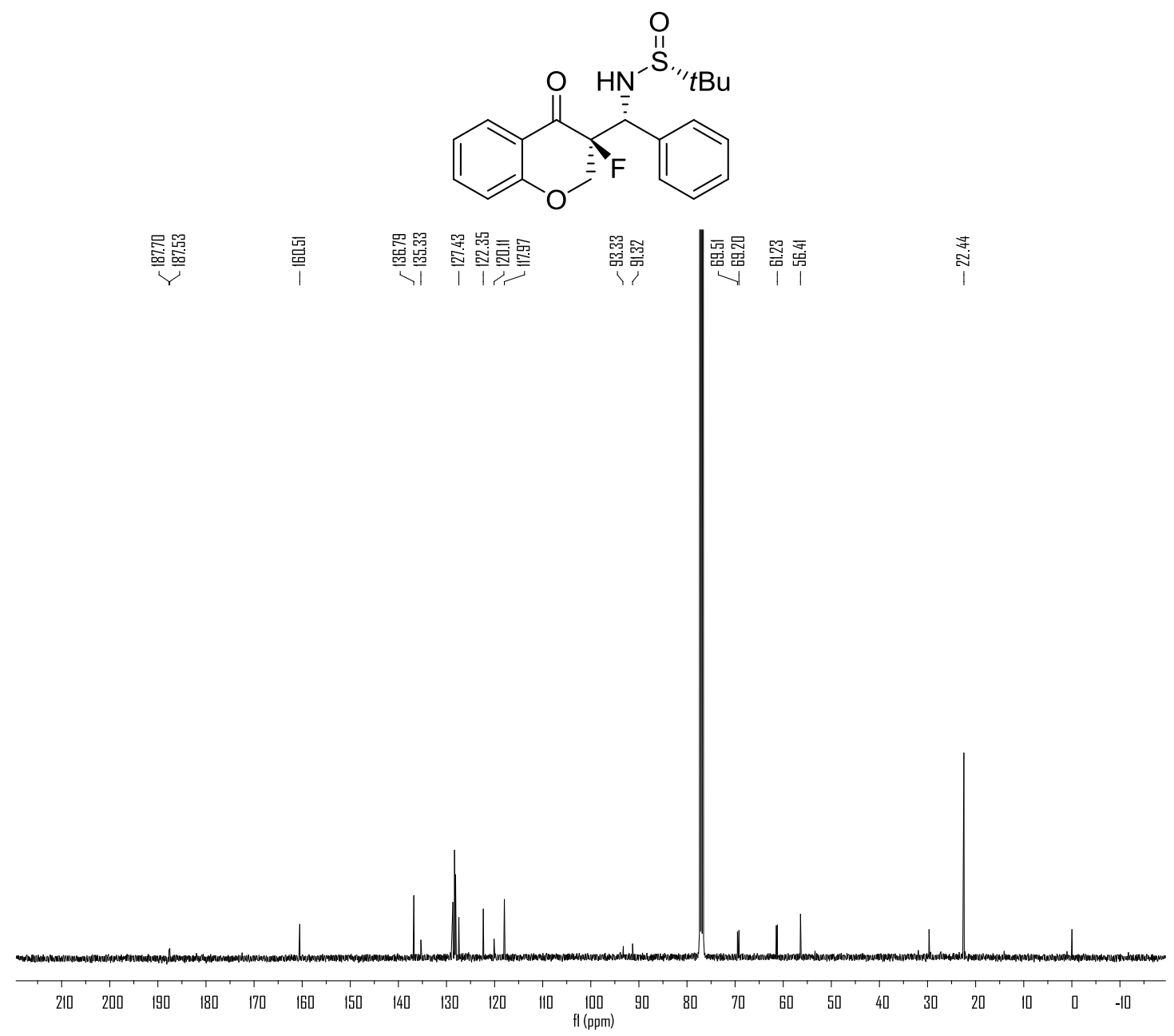


${ }^{1} \mathrm{H}$ NMR $\left(400 \mathrm{MHz}, \mathrm{CDCl}_{3}\right.$ ) spectrum of 3ca<smiles>O=C1c2ccsc2CCC1(NS(=O)[18O])c1ccccc1</smiles>

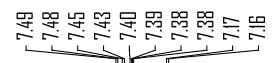

骂卧

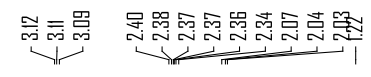

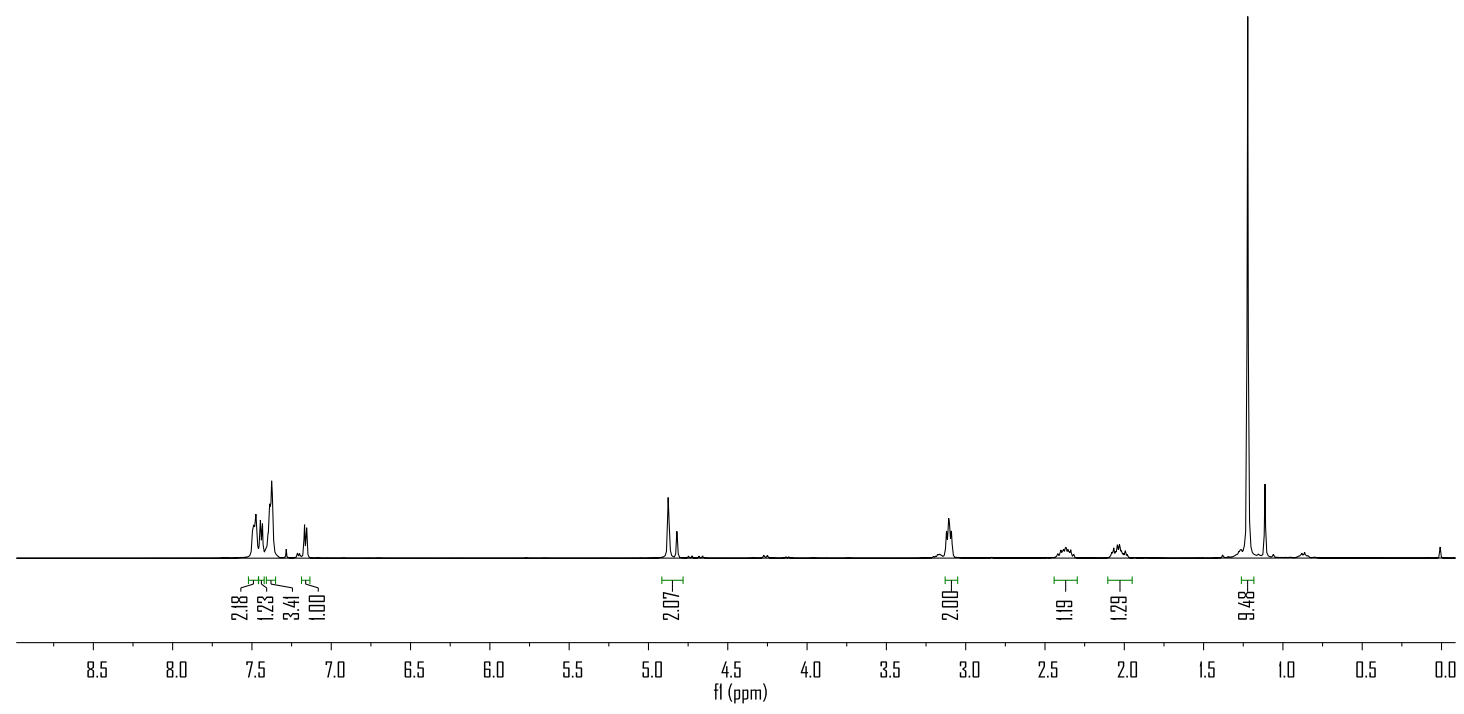


${ }^{19} \mathrm{~F}$ NMR $\left(376 \mathrm{MHz}, \mathrm{CDCl}_{3}\right.$ ) spectrum of 3ca<smiles>CC(C)(C)S(=O)N[C@H](c1ccccc1)C1(F)CCc2sccc2C1=O</smiles>

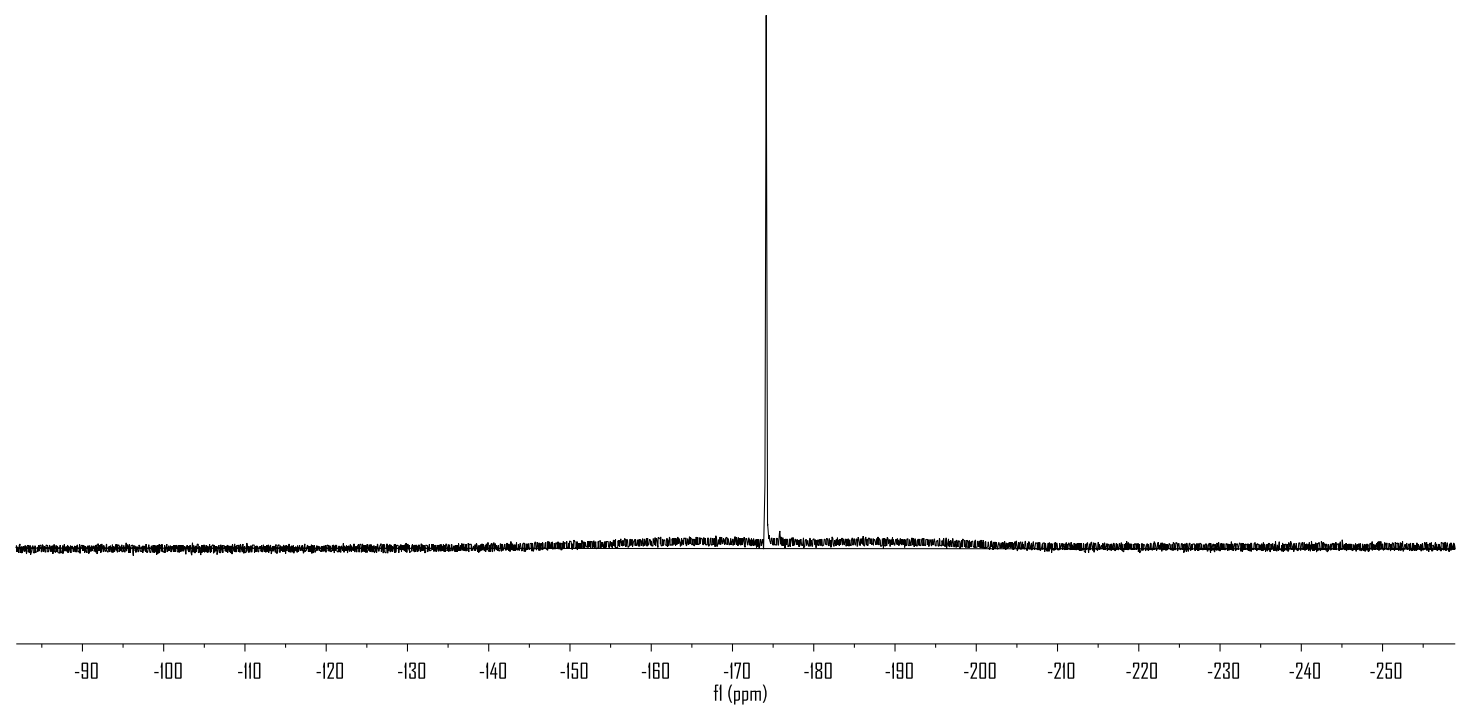


${ }^{13} \mathrm{C}$ NMR $\left(101 \mathrm{MHz}, \mathrm{CDCl}_{3}\right)$ spectrum of 3ca

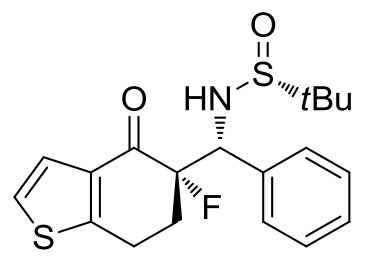

器品

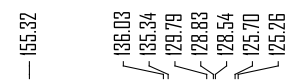

㞼早

器啰㗊

否哭
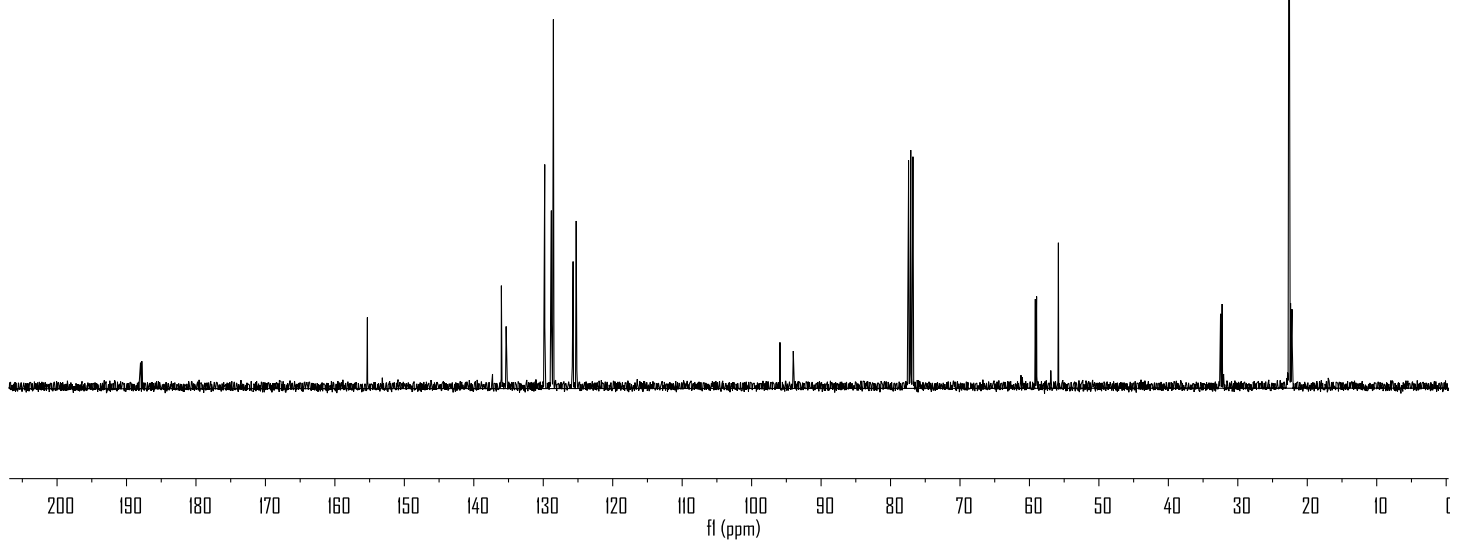

49 
${ }^{1} \mathrm{H}$ NMR (400 MHz, $\mathrm{CDCl}_{3}$ ) spectrum of 3ca'

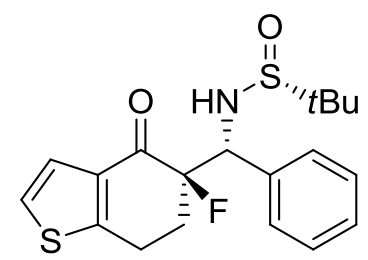

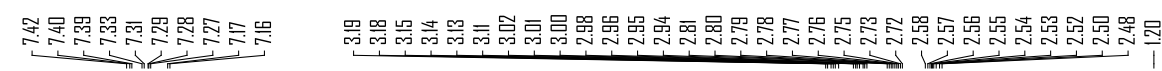

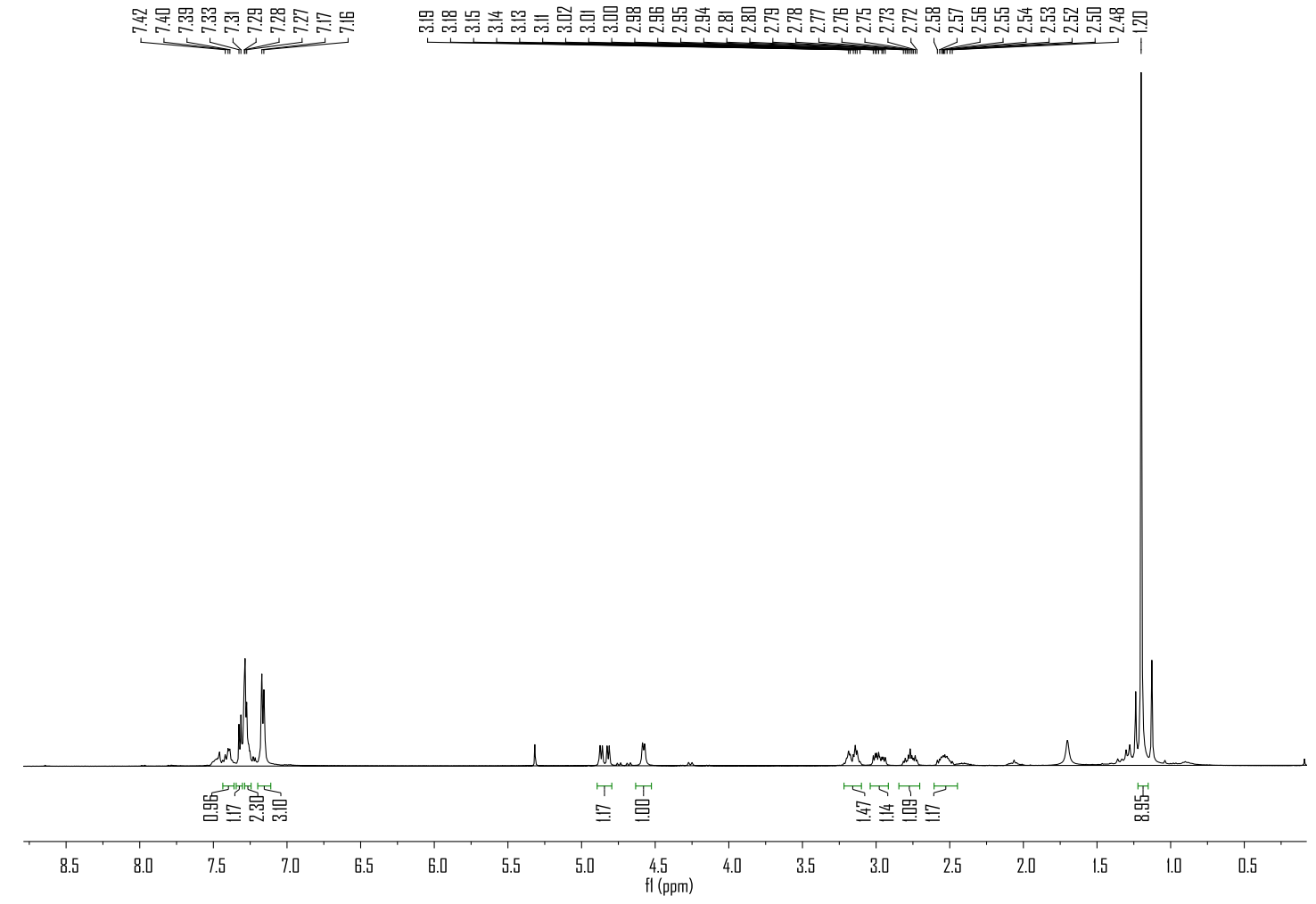


${ }^{19} \mathrm{~F} \mathrm{NMR}\left(376 \mathrm{MHz}, \mathrm{CDCl}_{3}\right)$ spectrum of 3ca'<smiles>CC(C)(C)S(=O)N[C@H](c1ccccc1)[C@@]1(F)CCc2sccc2C1=O</smiles>

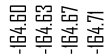

急

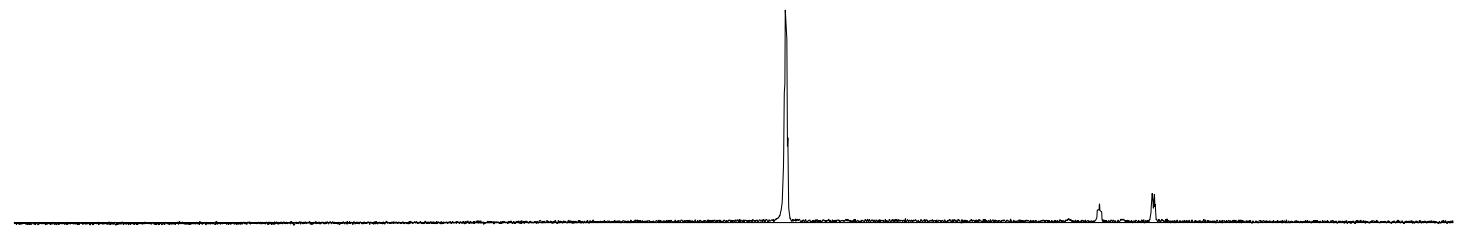

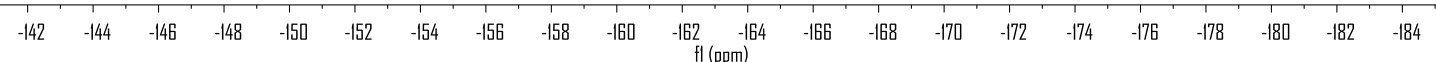


${ }^{13} \mathrm{C}$ NMR $\left(101 \mathrm{MHz}, \mathrm{CDCl}_{3}\right.$ ) spectrum of 3ca'

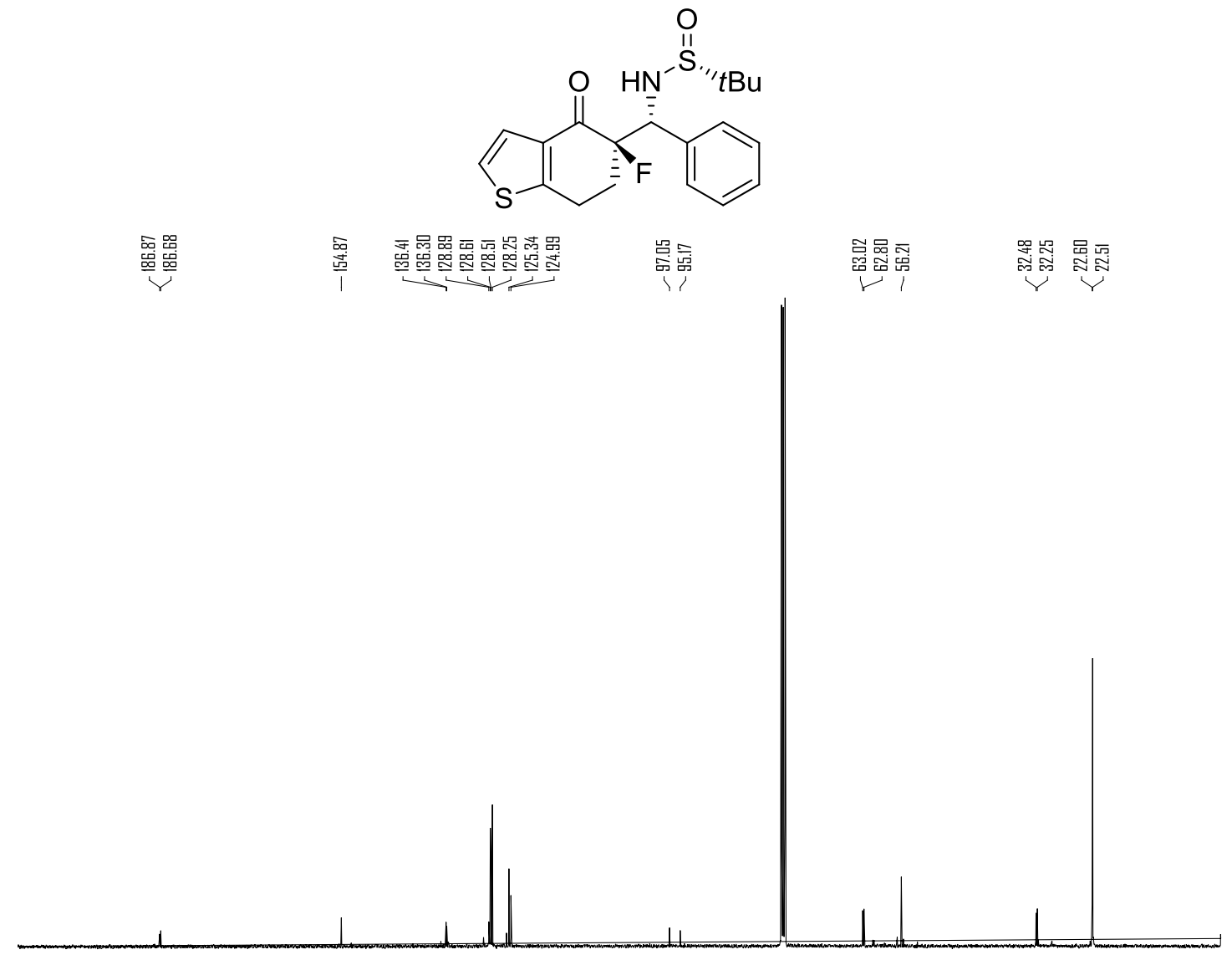

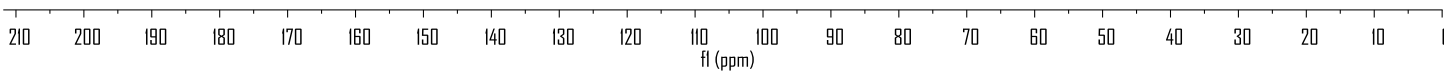


${ }^{1} \mathrm{H}$ NMR (400 MHz, $\mathrm{CDCl}_{3}$ ) spectrum of 3da

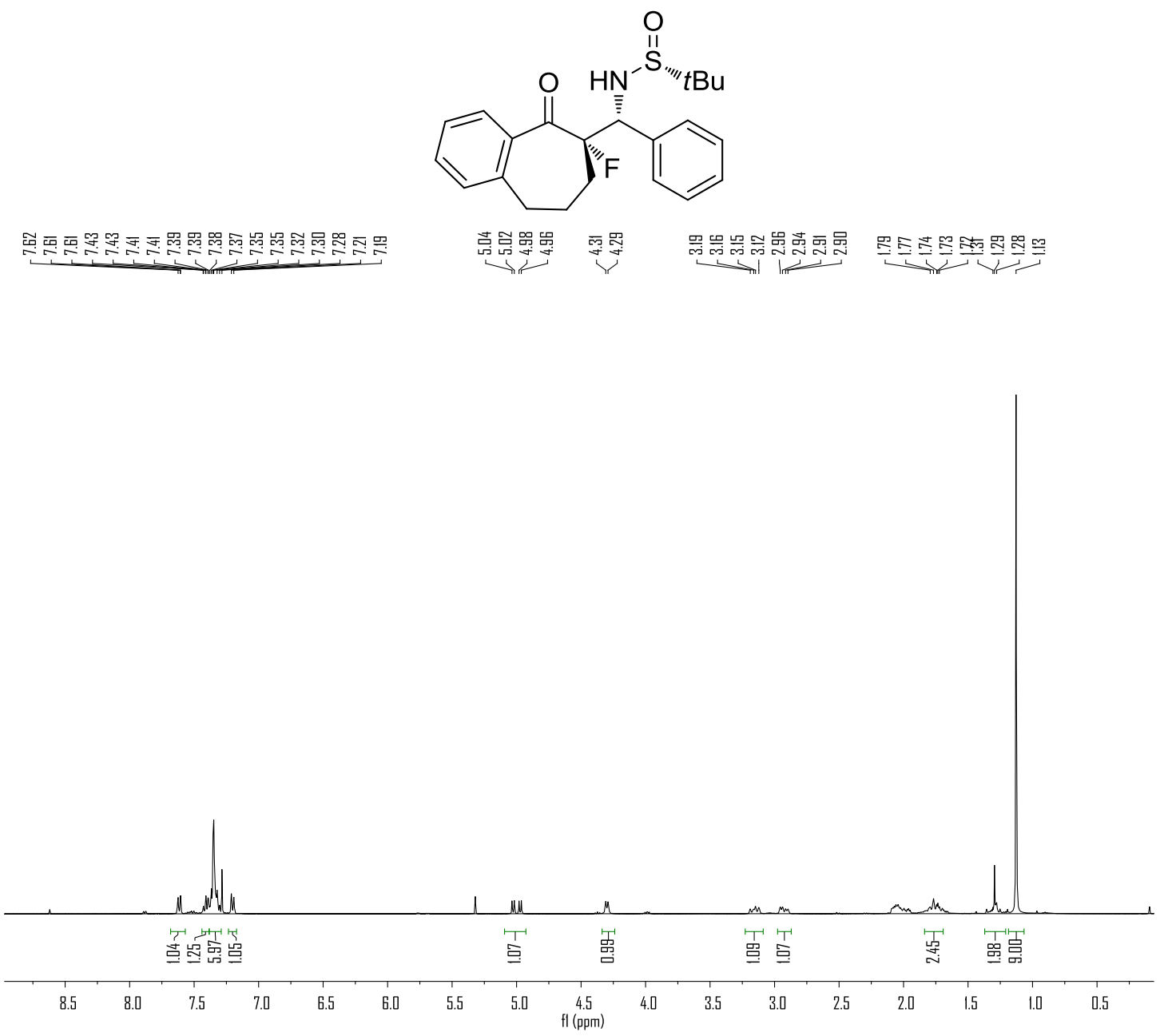


${ }^{19} \mathrm{~F}$ NMR $\left(376 \mathrm{MHz}, \mathrm{CDCl}_{3}\right)$ spectrum of $3 \mathbf{d a}$<smiles>CC(C)(C)S(=O)N[C@H](c1ccccc1)C1CCCc2ccccc2C1=O</smiles>

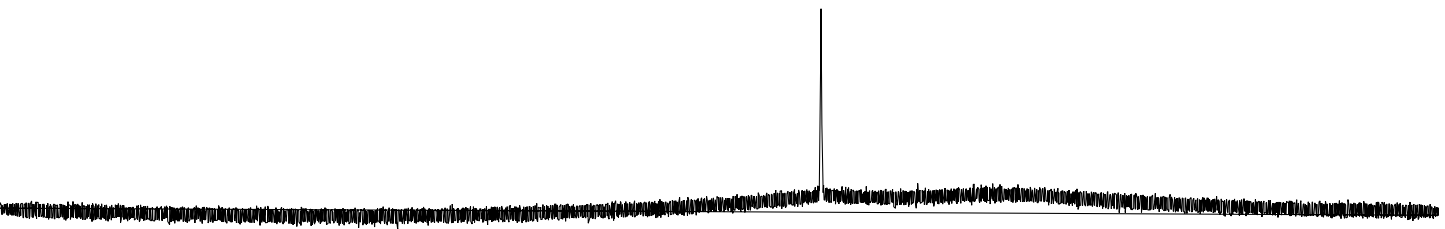

$\begin{array}{llllllllllllllllllllllll}-40 & -50 & -60 & -70 & -80 & -90 & -100 & -110 & -120 & -130 & -140 & -150 & -160 & -170 & -180 & -190 & -200 & -210 & -220 & -230 & -240 & -250 & -260\end{array}$ 
${ }^{13} \mathrm{C}$ NMR $\left(101 \mathrm{MHz}, \mathrm{CDCl}_{3}\right)$ spectrum of $\mathbf{3 d a}$<smiles>O=C1C(=O)c2ccccc2CCC=C1[C@H](N[S@@](=O)c1ccccc1)c1ccccc1</smiles>

嘀彎

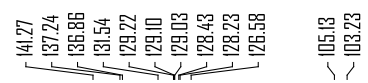

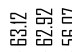

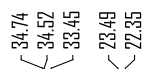

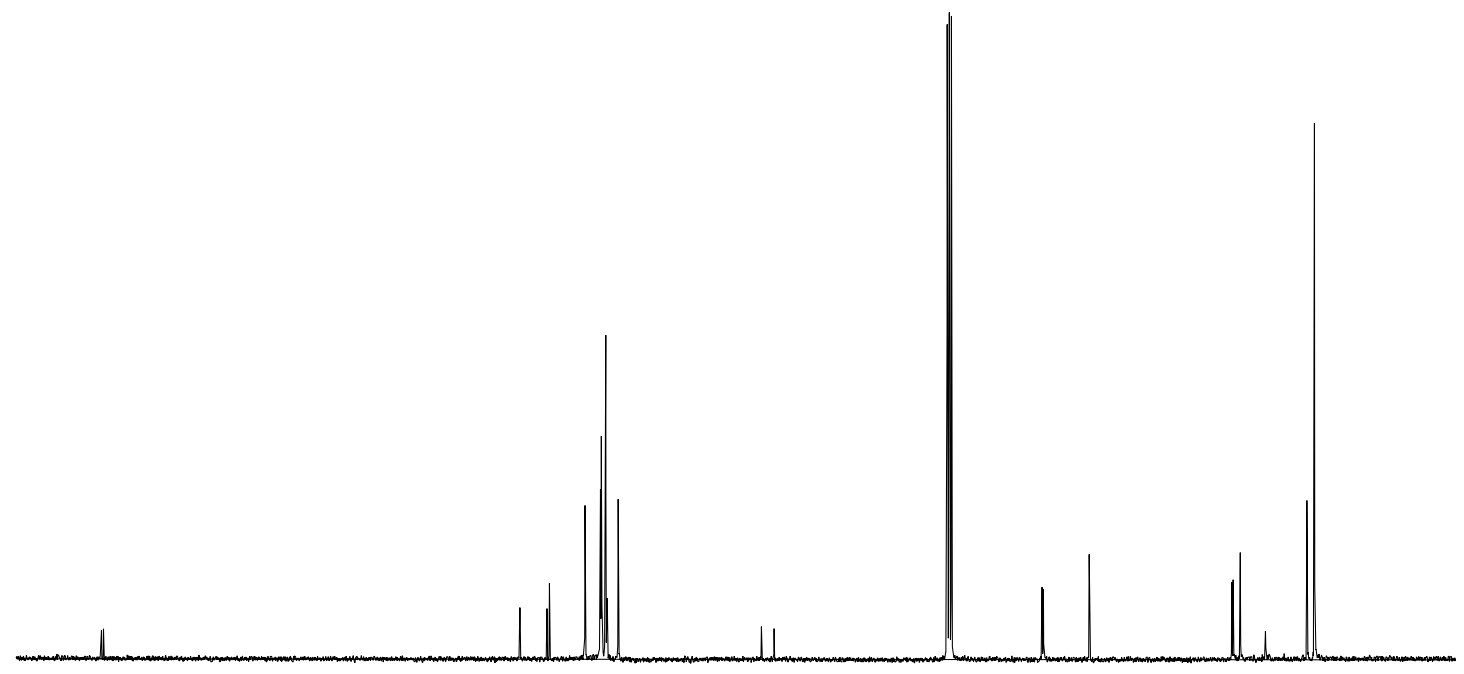

$\begin{array}{lllllllllllllllllllll}210 & 200 & 190 & 180 & 170 & 160 & 150 & 140 & 130 & 120 & 1100 & 100 & 90 & 80 & 70 & 60 & 50 & 40 & 30 & 20 & 10\end{array}$ 
${ }^{1} \mathrm{H}$ NMR (400 MHz, $\mathrm{CDCl}_{3}$ ) spectrum of 3ea<smiles>O=C1c2ccccc2CC1(NS(=O)[18O])c1ccccc1</smiles>

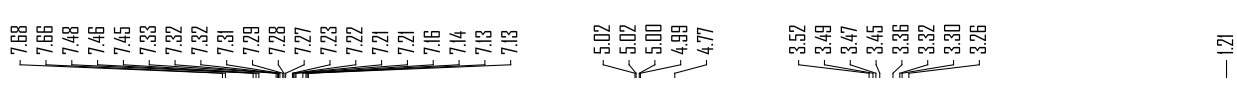

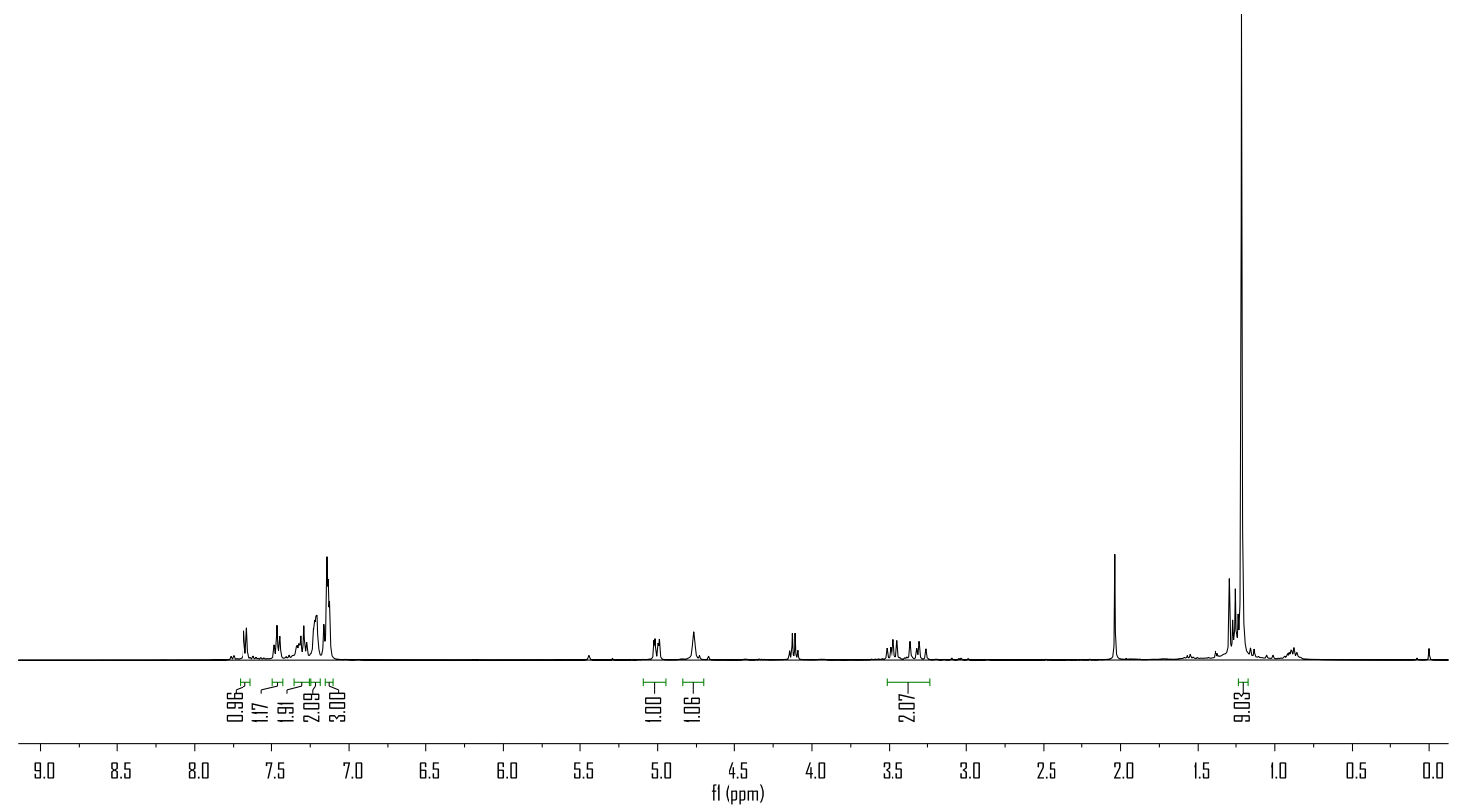


${ }^{19} \mathrm{~F}$ NMR $\left(376 \mathrm{MHz}, \mathrm{CDCl}_{3}\right.$ ) spectrum of $3 \mathbf{e a}$<smiles>CC(C)(C)S(=O)N[C@H](c1ccccc1)C1(F)Cc2ccccc2C1=O</smiles>

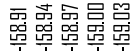

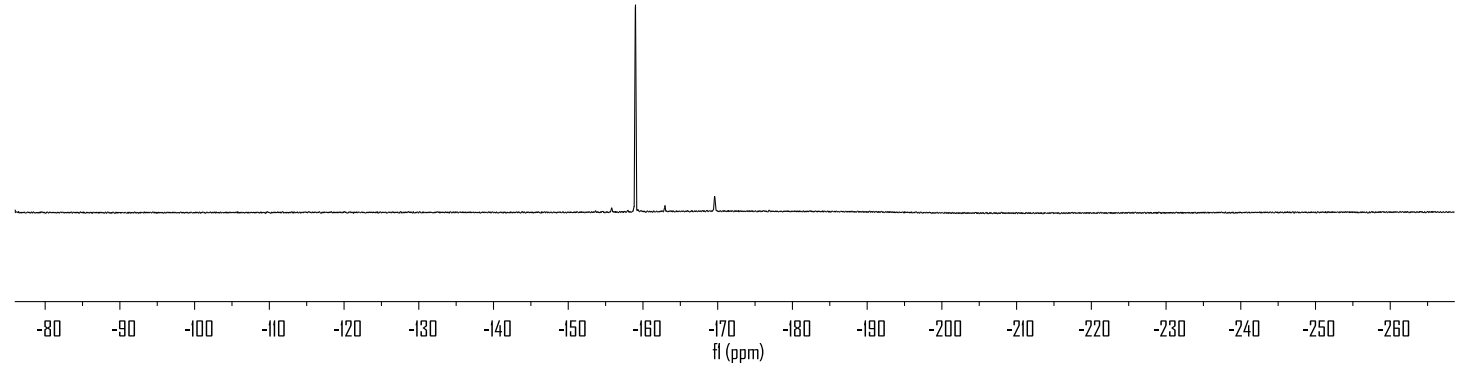


${ }^{13} \mathrm{C}$ NMR $\left(101 \mathrm{MHz}, \mathrm{CDCl}_{3}\right)$ spectrum of 3ea<smiles>CC(C)(C)S(=O)N[C@H](c1ccccc1)C1(F)Cc2ccccc2C1=O</smiles>

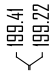

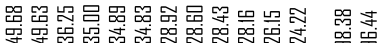

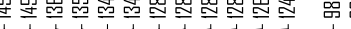

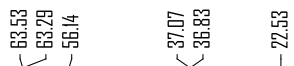

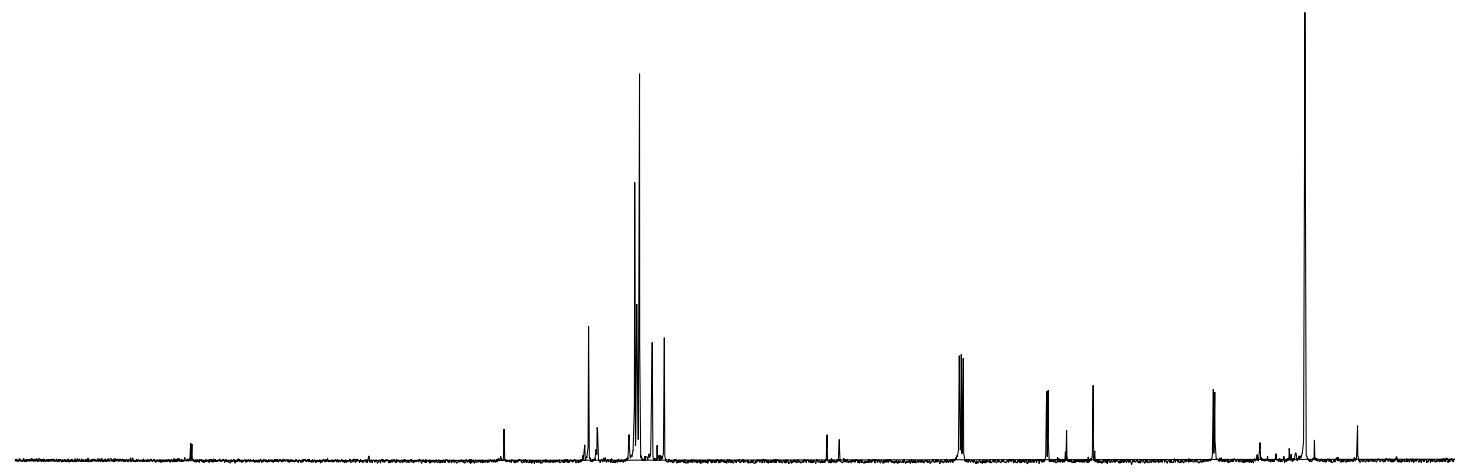

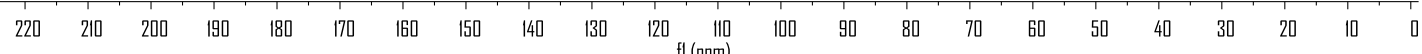


${ }^{1} \mathrm{H}$ NMR (400 MHz, $\mathrm{CDCl}_{3}$ ) spectrum of $\mathbf{3 f a}$<smiles>O=C1Cc2ccccc2C1(N[S@](=O)[18O])c1ccccc1</smiles>

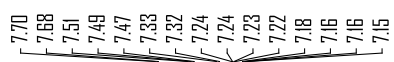

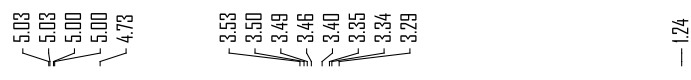

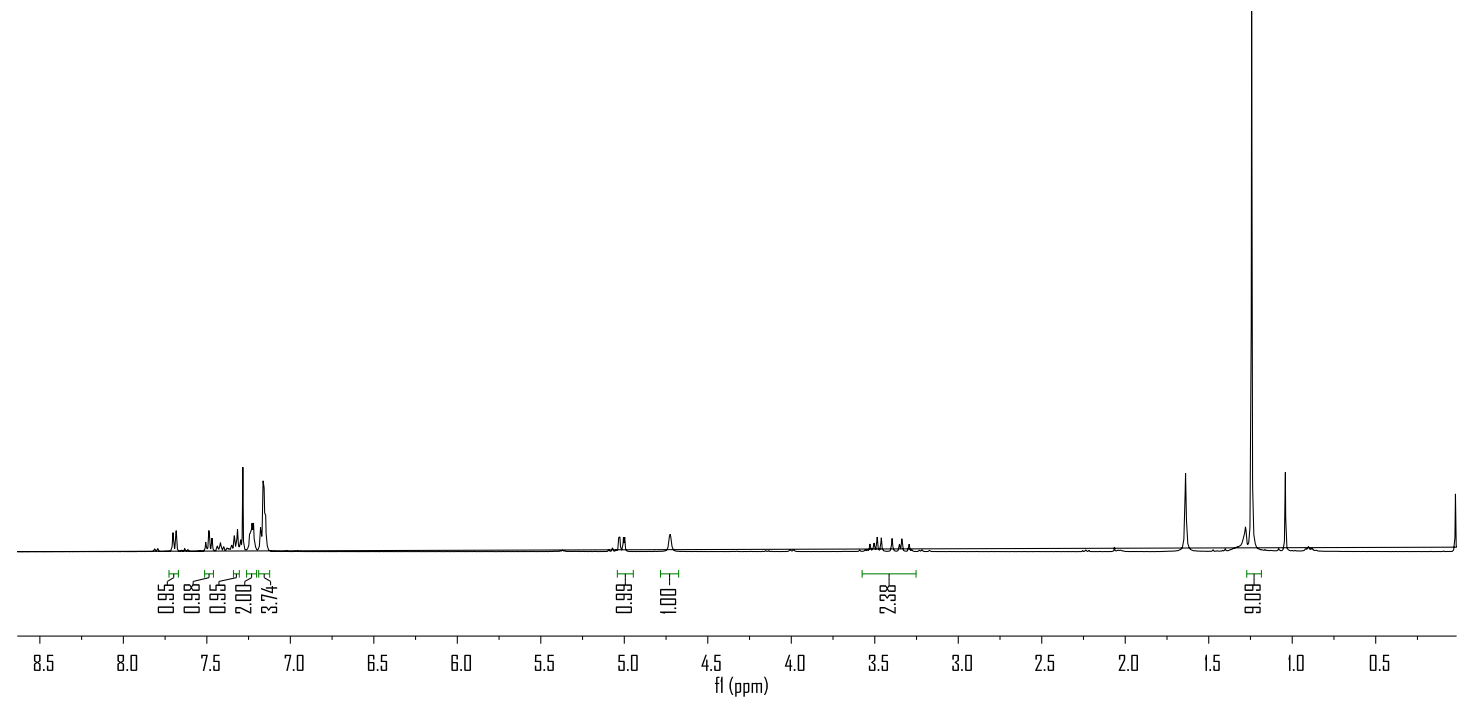


${ }^{19} \mathrm{~F}$ NMR $\left(376 \mathrm{MHz}, \mathrm{CDCl}_{3}\right)$ spectrum of $\mathbf{3 f a}$<smiles>CC(C)(C)S(=O)N[C@H](c1ccccc1)C1(F)C(=O)Cc2ccccc21</smiles> 
${ }^{13} \mathrm{C}$ NMR (101 MHz, $\mathrm{CDCl}_{3}$ ) spectrum of $\mathbf{3 f a}$

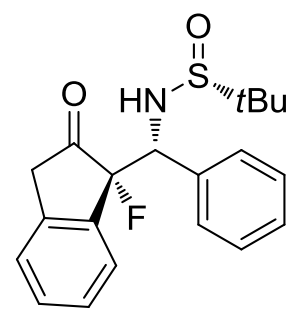

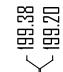

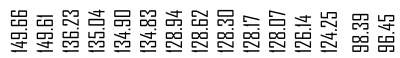

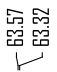

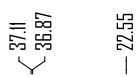

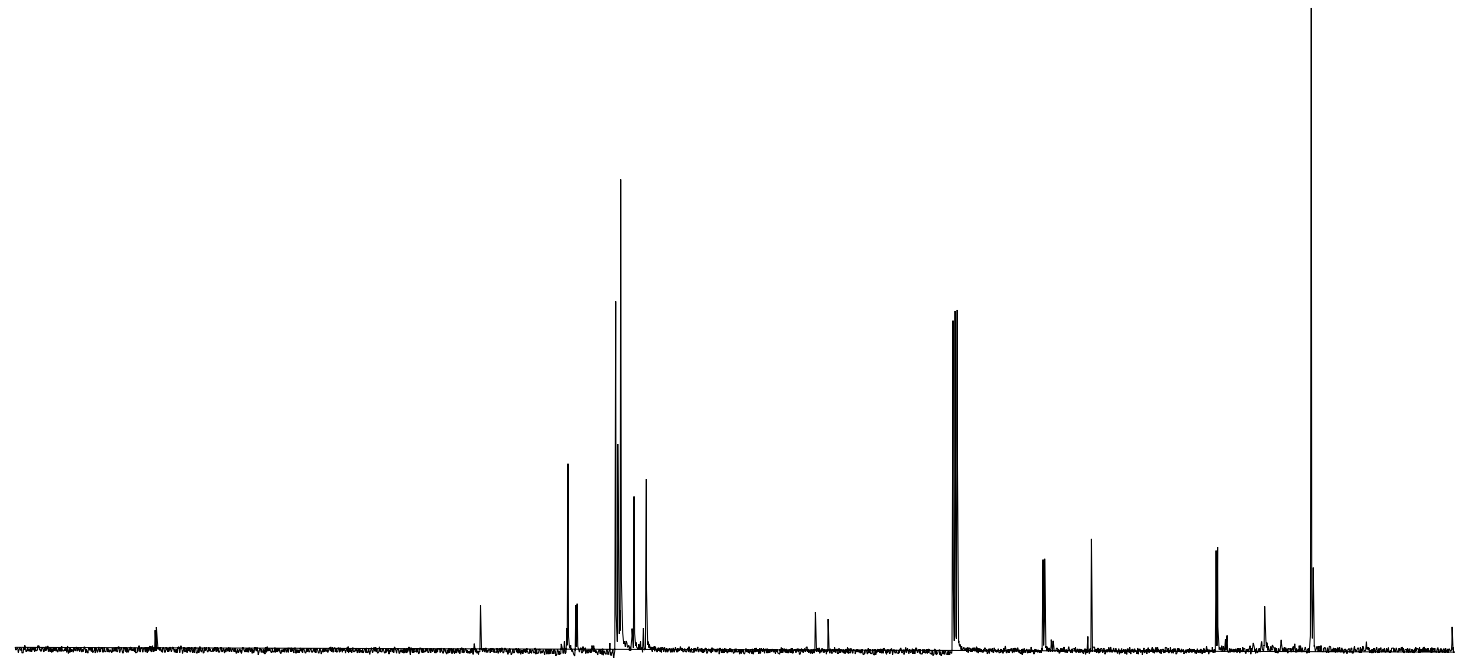

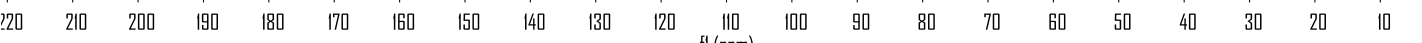


${ }^{1} \mathrm{H}$ NMR (400 MHz, $\mathrm{CDCl}_{3}$ ) spectrum of $\mathbf{3 g b}$<smiles>Cc1ccc(C(N[S@](=O)[O-])C(C)(C)C(=O)c2ccccc2)cc1</smiles>

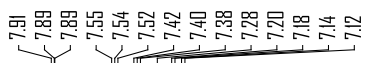

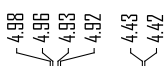

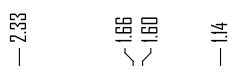

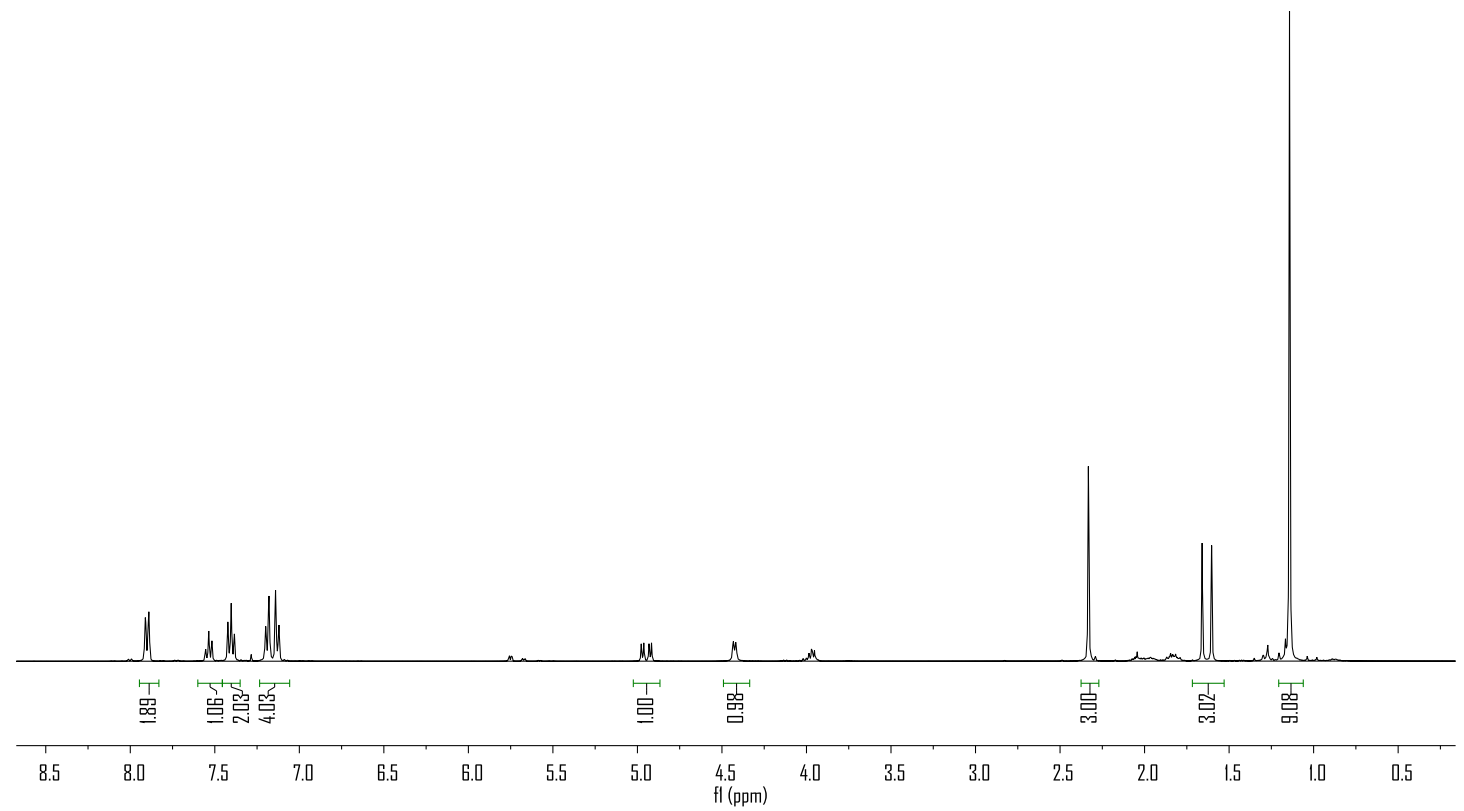


${ }^{19} \mathrm{~F}$ NMR (376 MHz, $\mathrm{CDCl}_{3}$ ) spectrum of $\mathbf{3 g b}$
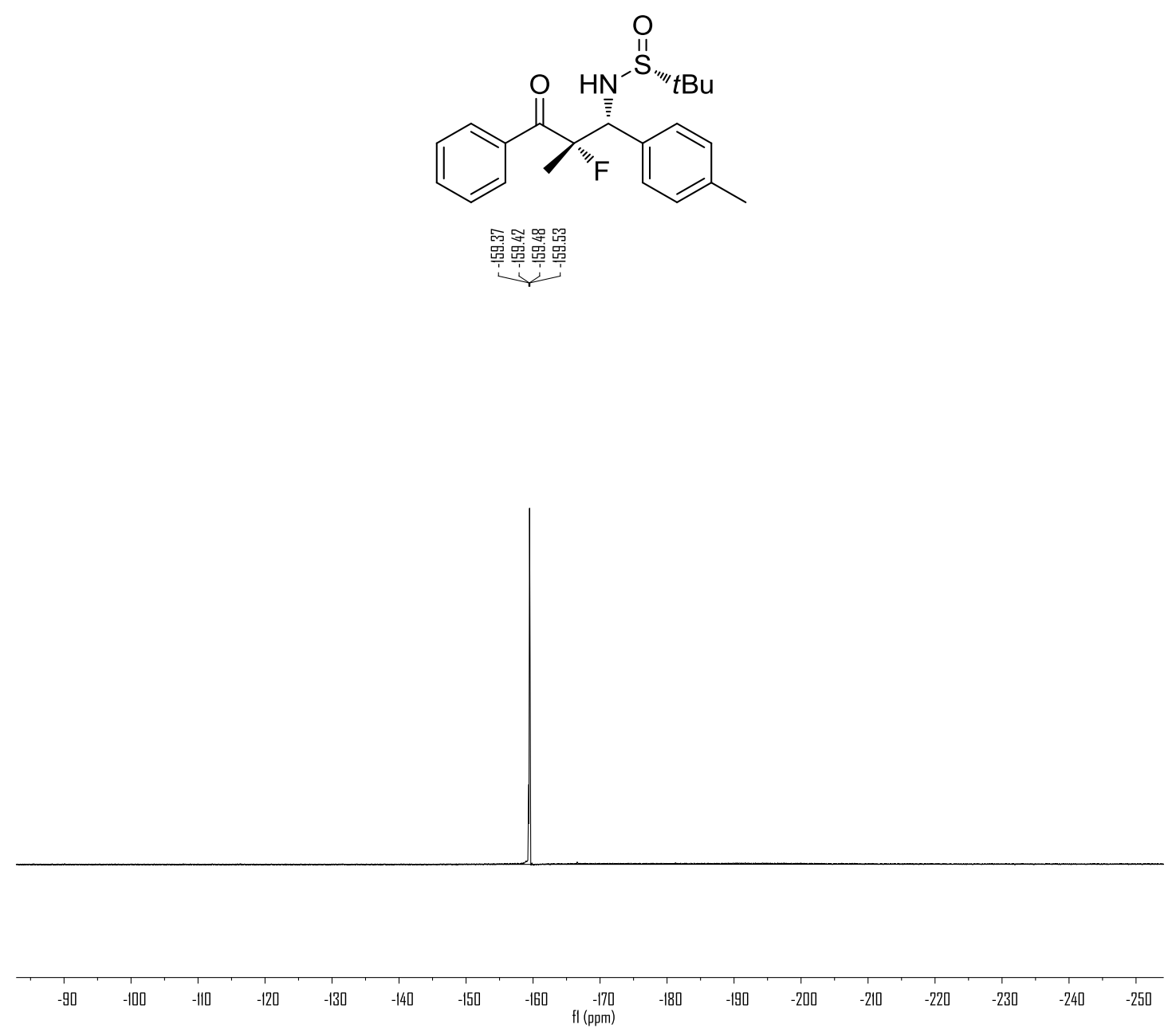
${ }^{13} \mathrm{C}$ NMR (101 MHz, $\mathrm{CDCl}_{3}$ ) spectrum of $\mathbf{3 g b}$<smiles>Cc1ccc(C(NS(=O)C(=O)c2ccccc2)C(C)(C)C(=O)c2ccccc2)cc1</smiles>

衰

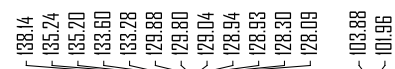

器器嵒

罚罚罚志
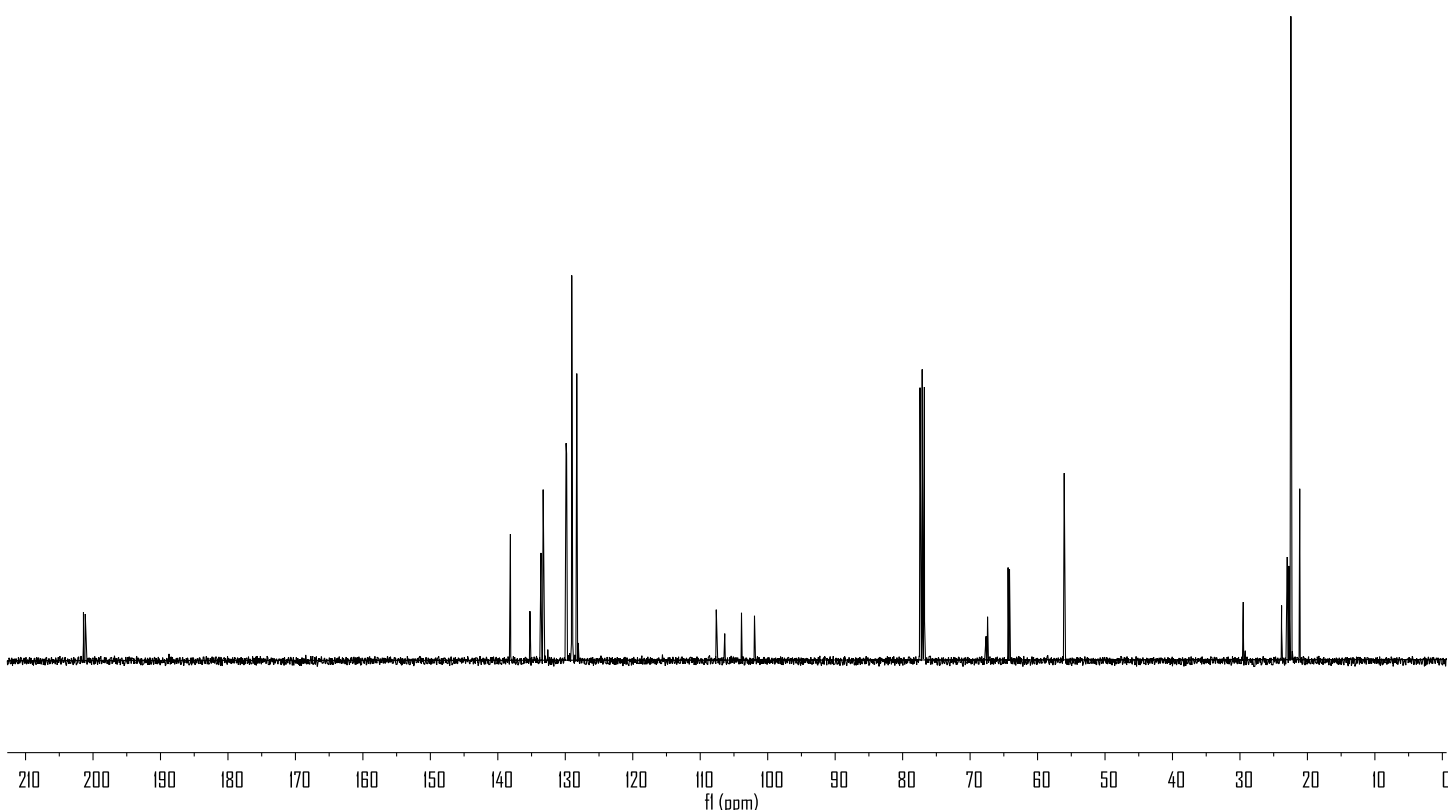
${ }^{1} \mathrm{H}$ NMR $\left(400 \mathrm{MHz}, \mathrm{CDCl}_{3}\right)$ spectrum of $\mathbf{3 h a}$<smiles>O=C([18O])[C@@H](NC(=O)C12CC3CC(CC(C3)C1)C2)c1ccccc1</smiles>

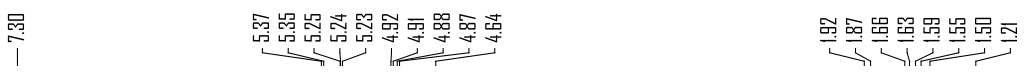

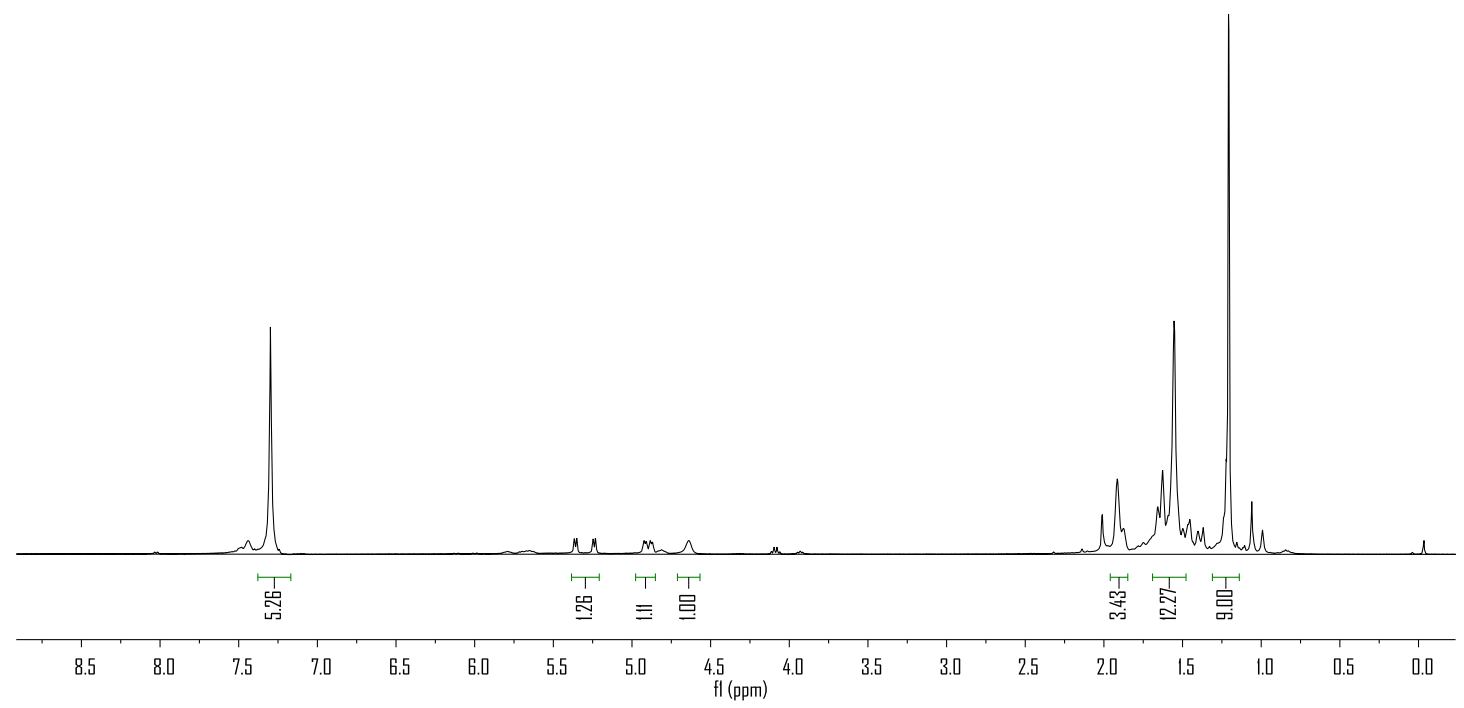


${ }^{19} \mathrm{~F}$ NMR $\left(376 \mathrm{MHz}, \mathrm{CDCl}_{3}\right)$ spectrum of 3ha<smiles>CC(C)(C)S(=O)N[C@H](c1ccccc1)[C@H](F)C(=O)C12CC3CC(CC(C3)C1)C2</smiles>

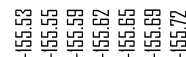

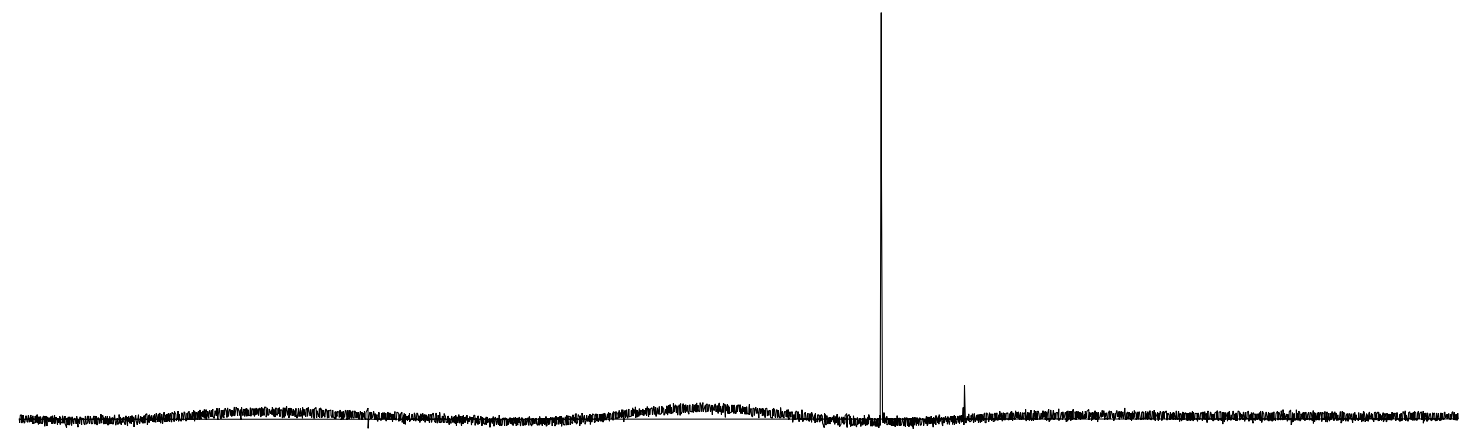


${ }^{13} \mathrm{C}$ NMR $\left(101 \mathrm{MHz}, \mathrm{CDCl}_{3}\right)$ spectrum of $3 \mathbf{h a}$<smiles>O=C(N[C@H](c1ccccc1)C(F)C12CC3CC(CC(C3)C1)C2)[13C](=O)[O-]</smiles>

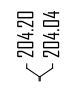

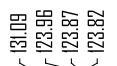

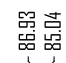

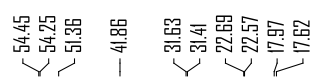

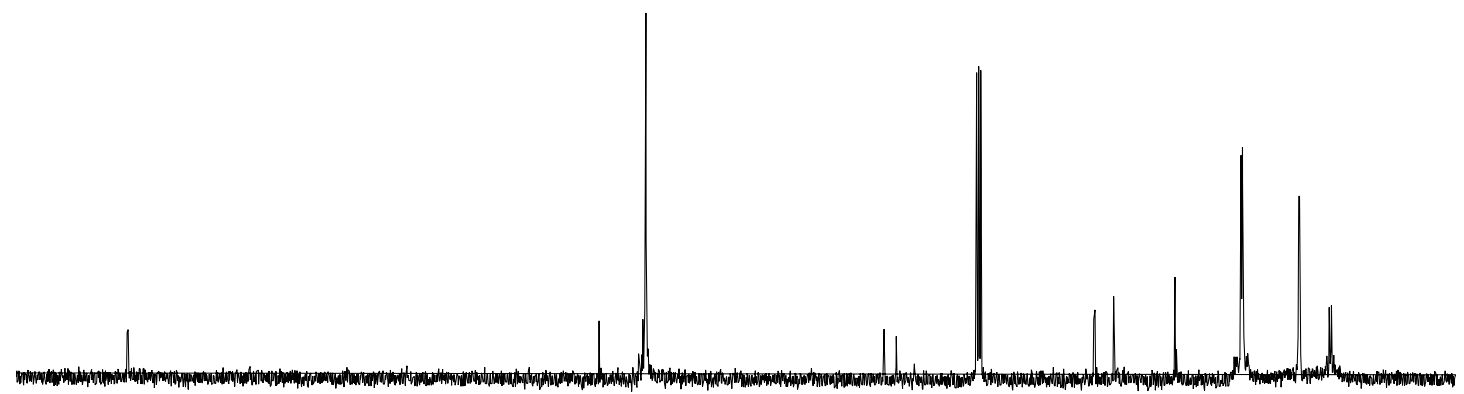

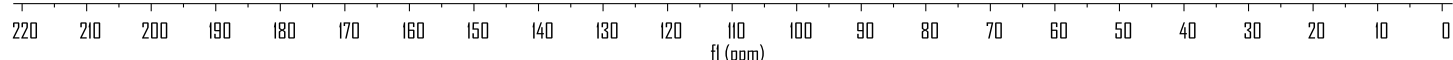


${ }^{1} \mathrm{H}$ NMR (400 MHz, $\mathrm{CDCl}_{3}$ ) spectrum of 3ib<smiles>Cc1ccc(C(NS(=O)C(=O)c2ccccc2)C(F)C(=O)c2ccccc2C)cc1</smiles>

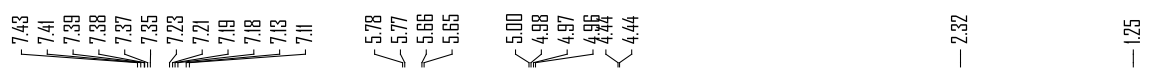

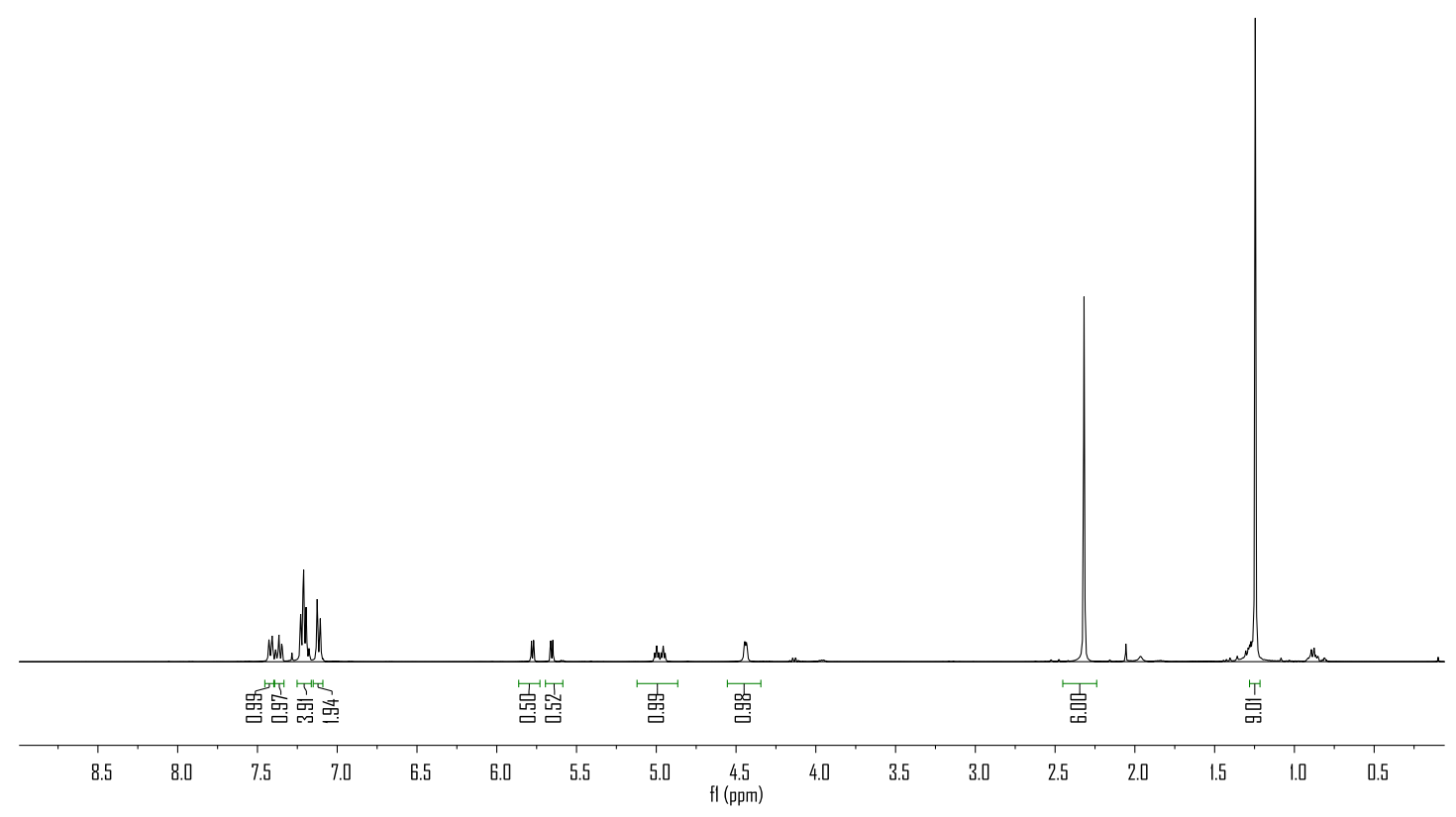


${ }^{19} \mathrm{~F}$ NMR (376 MHz, $\mathrm{CDCl}_{3}$ ) spectrum of Bib
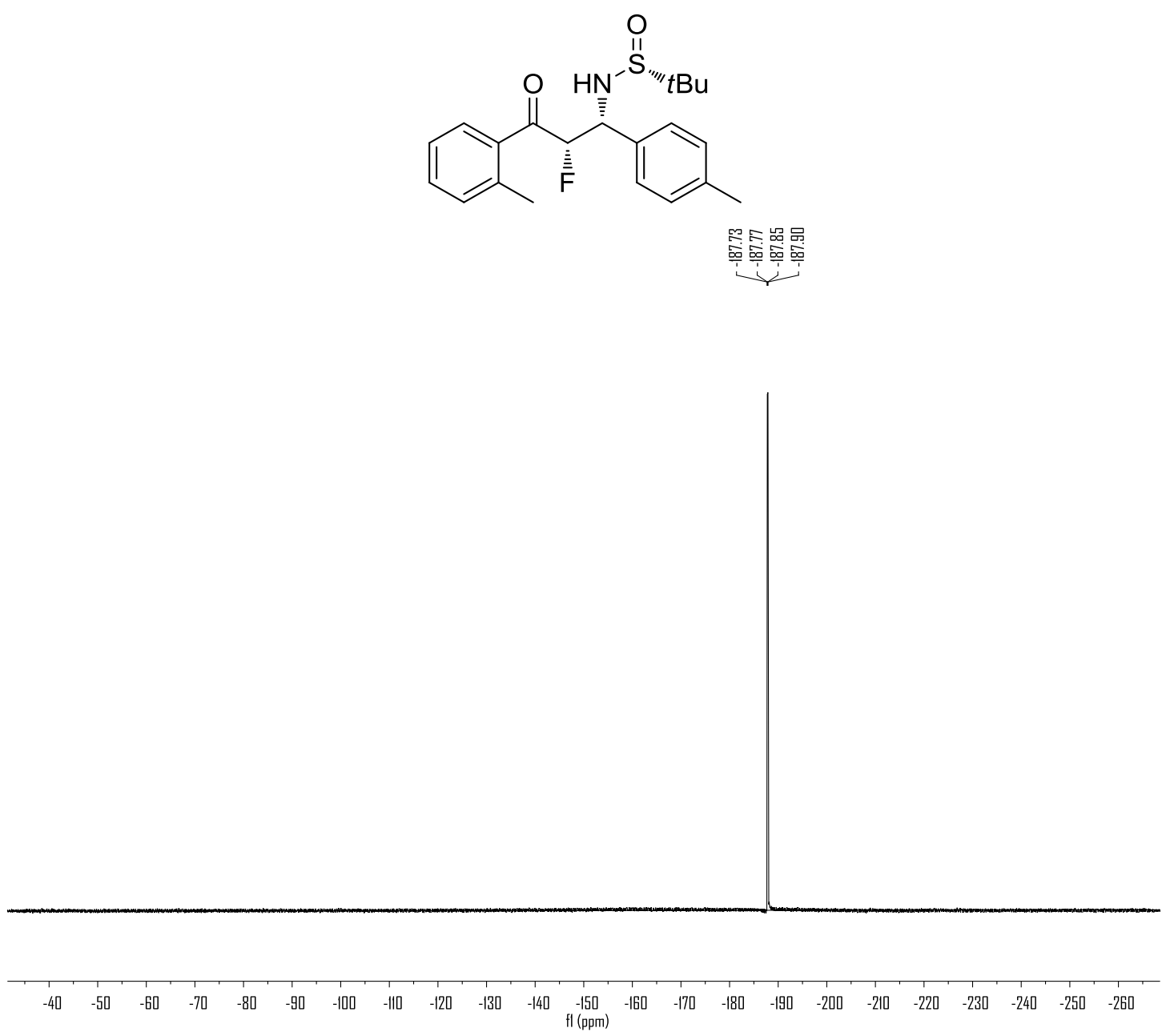

69 
${ }^{13} \mathrm{C}$ NMR (101 MHz, $\mathrm{CDCl}_{3}$ ) spectrum of 3ib<smiles>Cc1ccc(C(NS(=O)C(=O)c2ccccc2)C(F)C(=O)c2ccccc2C)cc1</smiles>

㽬黄

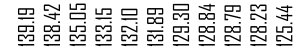

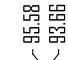

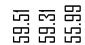

怘虽品

$\checkmark$

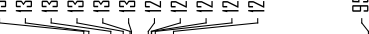

Y'

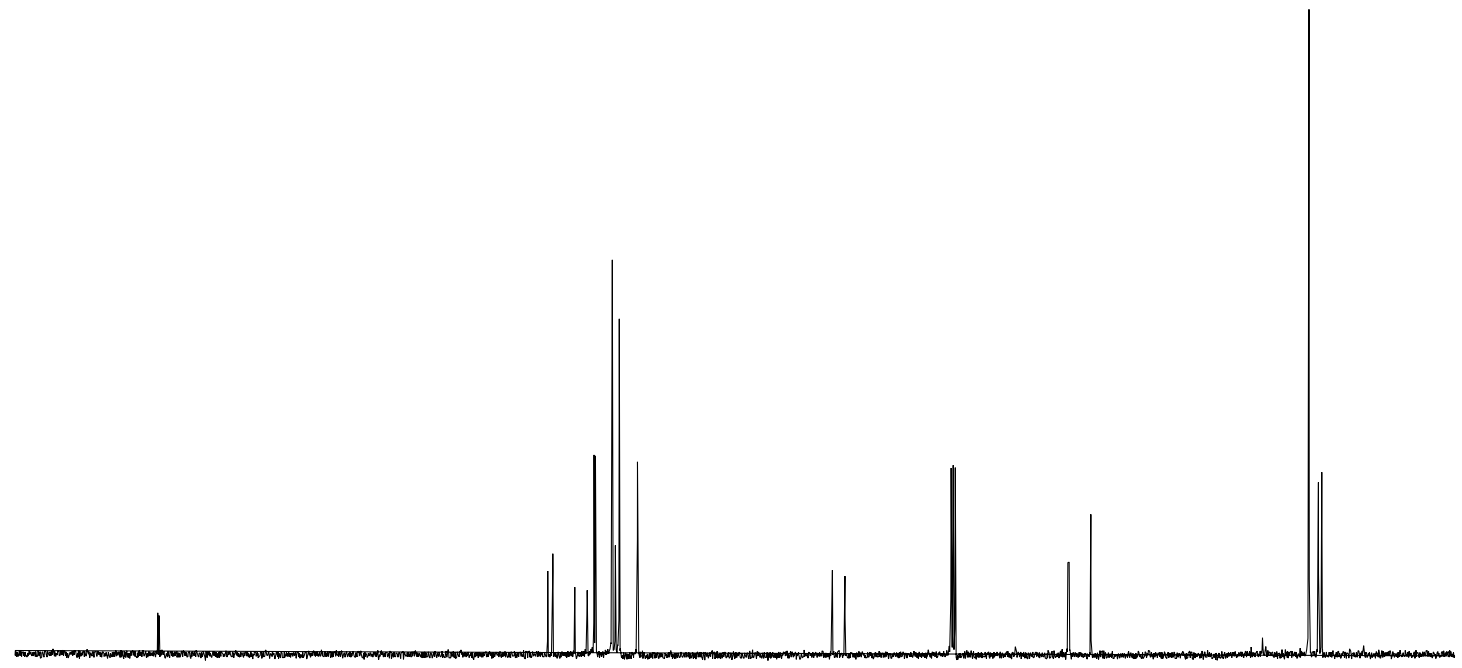

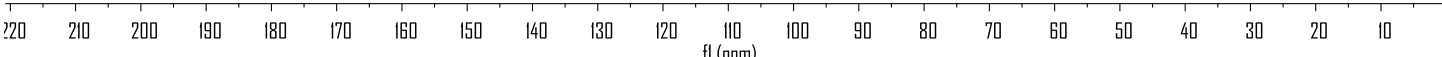


${ }^{1} \mathrm{H}$ NMR (400 MHz, $\mathrm{CDCl}_{3}$ ) spectrum of $\mathbf{3 j b}$<smiles>COc1ccccc1C(=O)C(F)[C@H](NS(=O)C(=O)c1ccccc1)c1ccc(C)cc1</smiles>

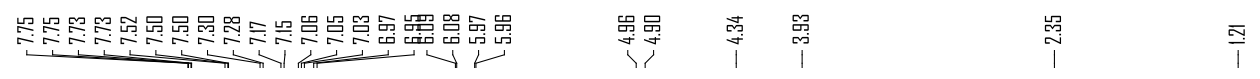

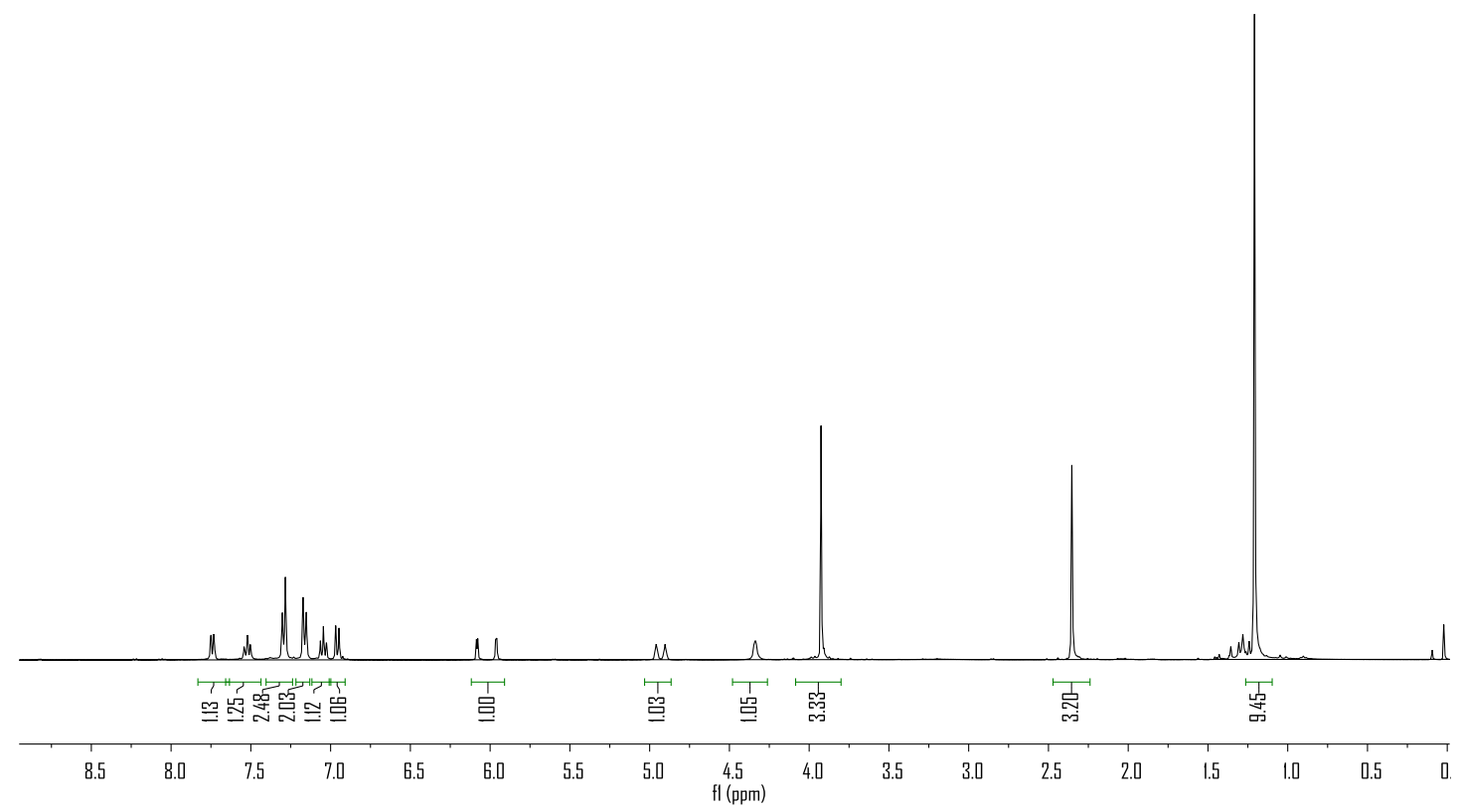


${ }^{19} \mathrm{~F}$ NMR (376 MHz, $\mathrm{CDCl}_{3}$ ) spectrum of $\mathbf{3 j b}$<smiles>COc1ccccc1C(=O)C(F)[C@H](NS(=O)[O-])c1ccc(C)cc1</smiles>

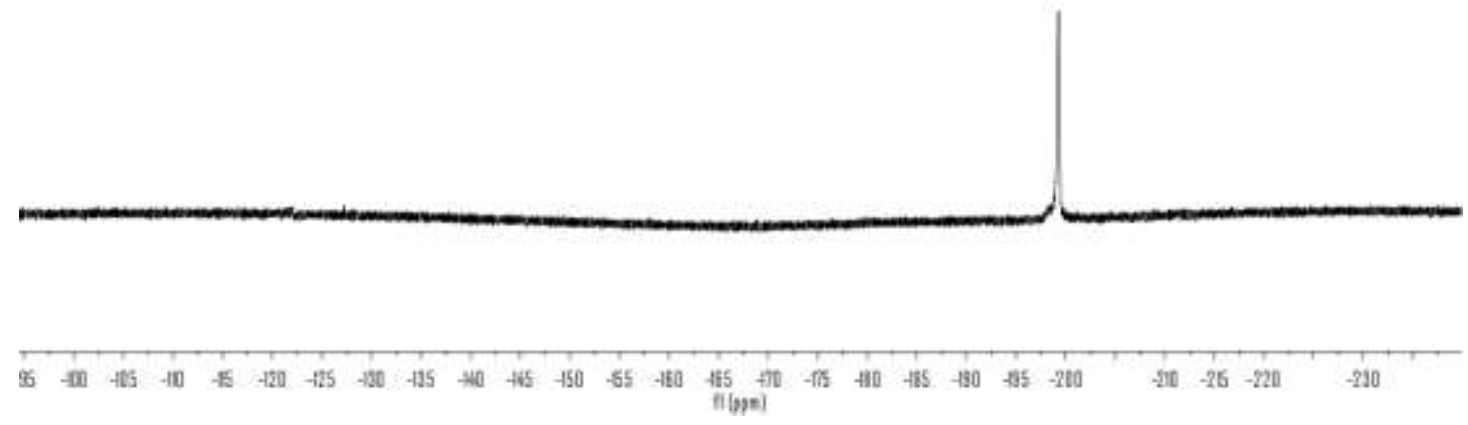


${ }^{13} \mathrm{C}$ NMR (101 MHz, $\left.\mathrm{CDCl}_{3}\right)$ spectrum of $\mathbf{3 j b}$<smiles>COc1ccccc1C(=O)[C@H](F)[C@@H](NS(=O)C(C)(C)C)c1ccc(C)cc1</smiles>

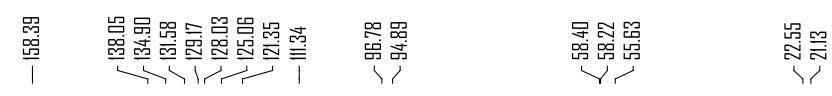

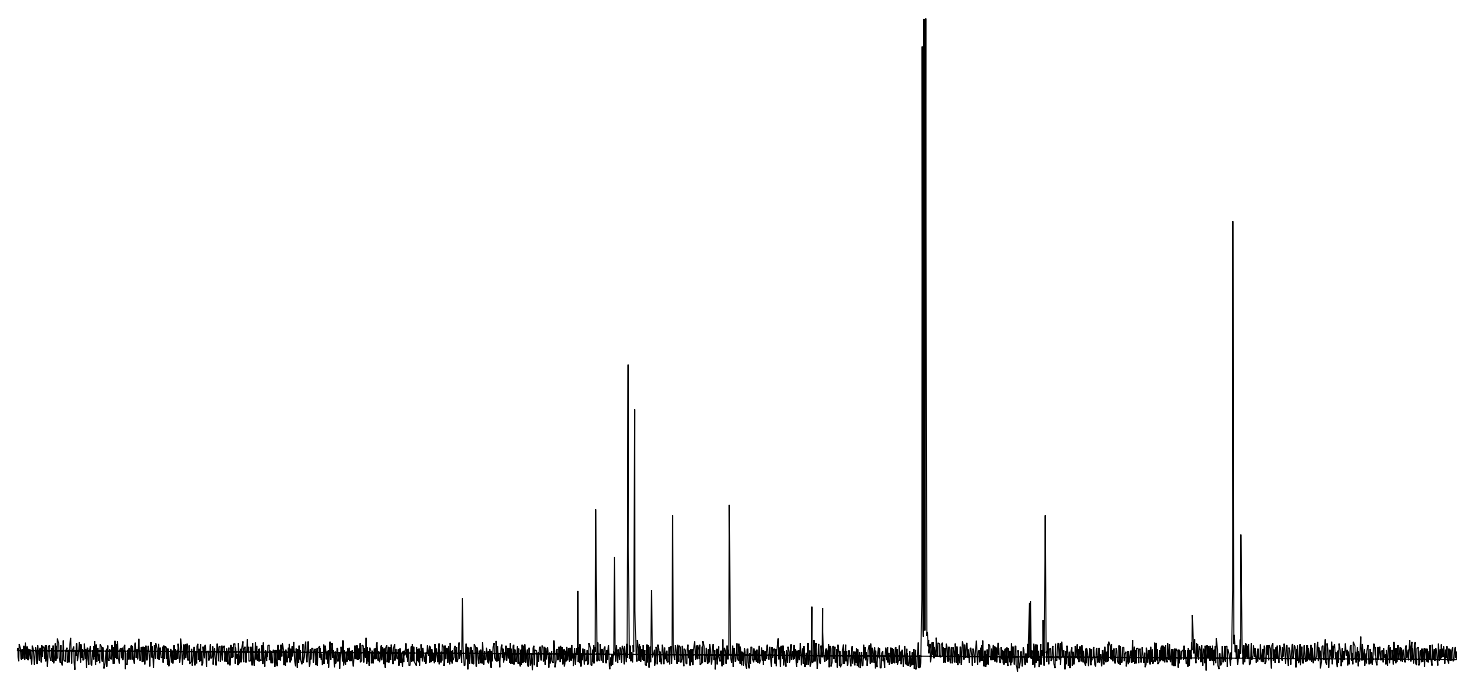

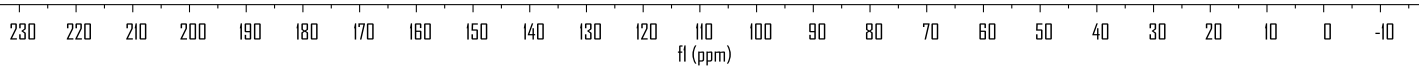


${ }^{1} \mathrm{H}$ NMR (400 MHz, $\mathrm{CDCl}_{3}$ ) spectrum of $\mathbf{3 a b}$<smiles>CC(=O)N[C@H](c1ccc(C)cc1)C1CCc2ccccc2C1=O</smiles>

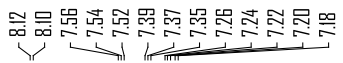

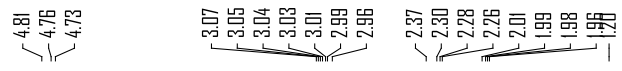

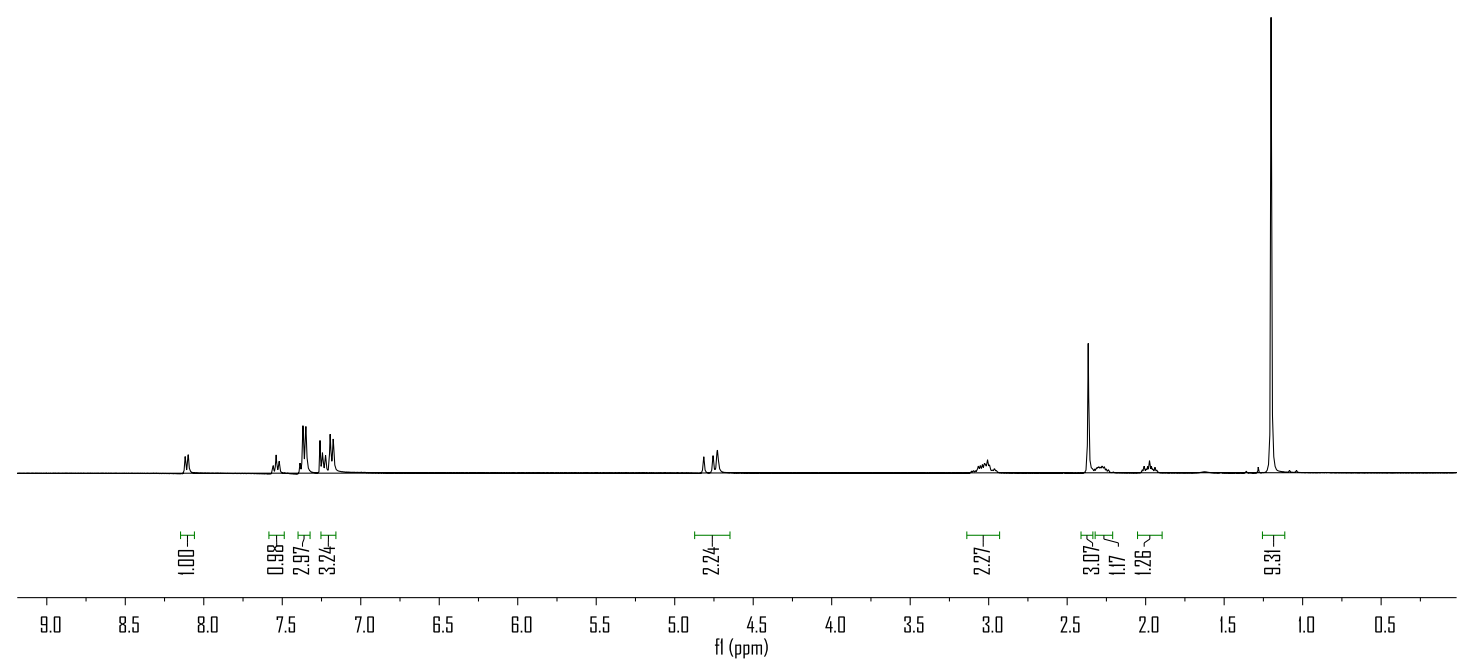


${ }^{19} \mathrm{~F}$ NMR (376 MHz, $\mathrm{CDCl}_{3}$ ) spectrum of 3ab<smiles>CC(=O)SN[C@H](c1ccc(C)cc1)[C@H]1[CH+]Cc2ccccc2C1=O</smiles>

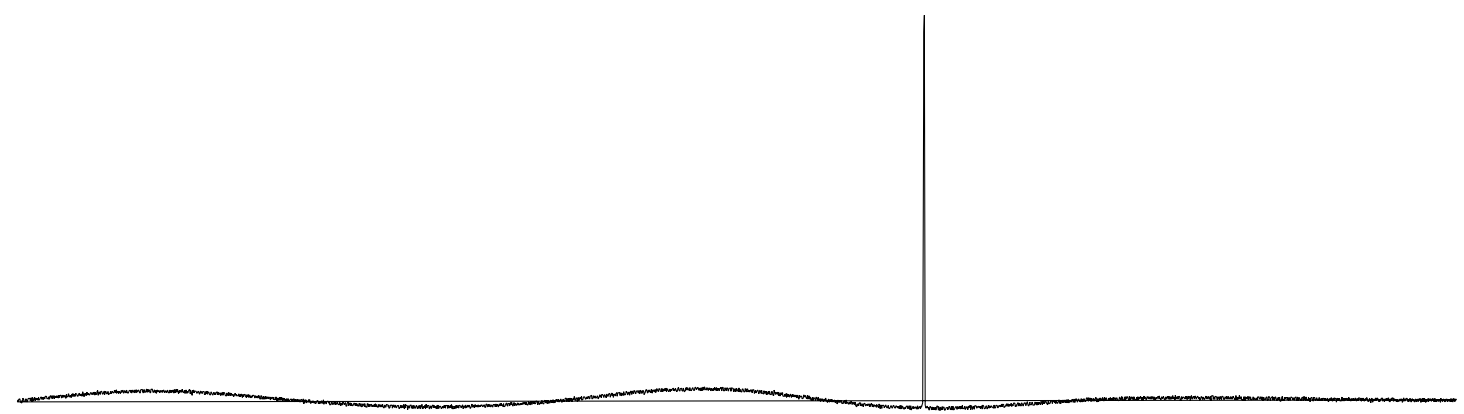


${ }^{13} \mathrm{C}$ NMR (101 MHz, $\mathrm{CDCl}_{3}$ ) spectrum of 3ab

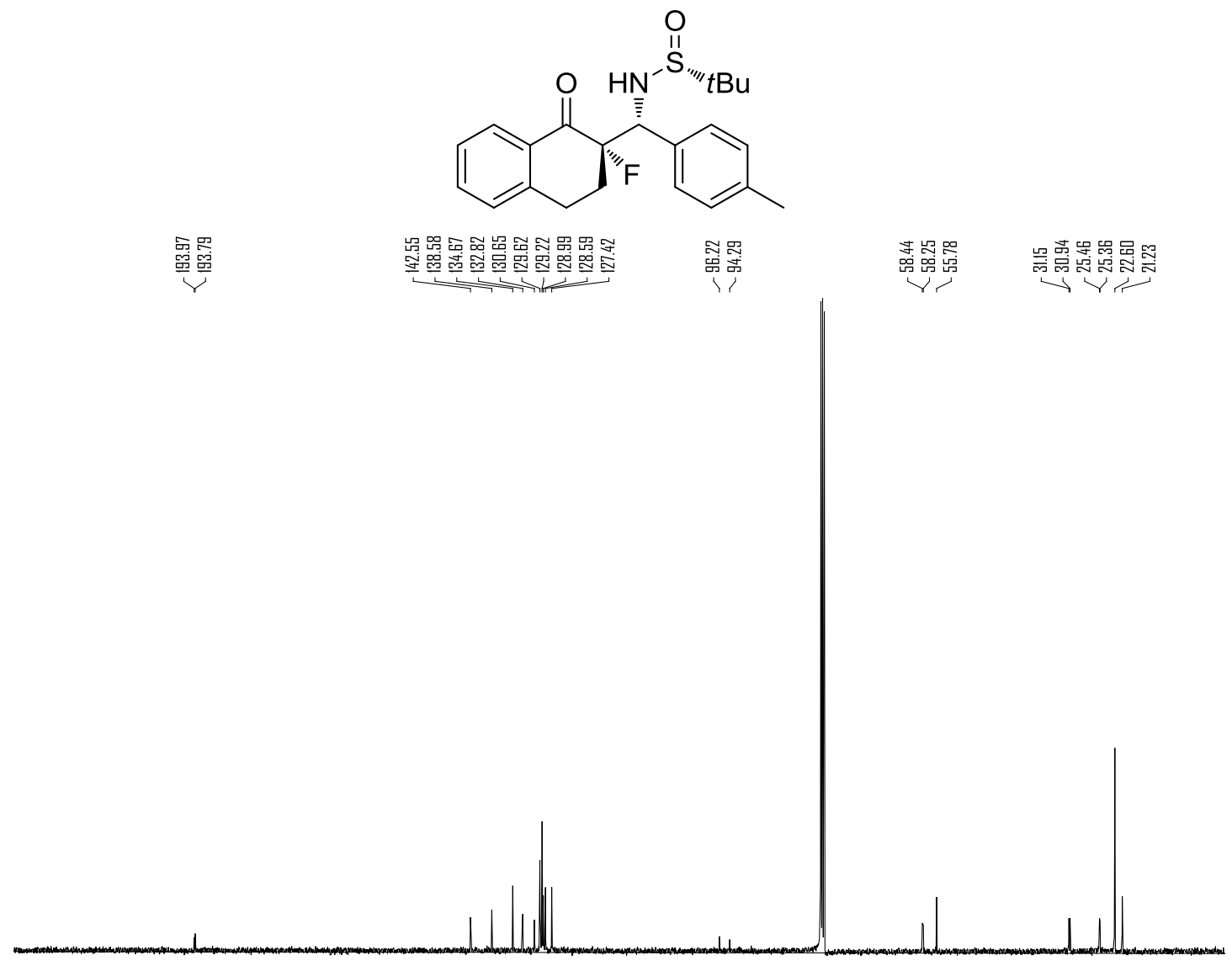

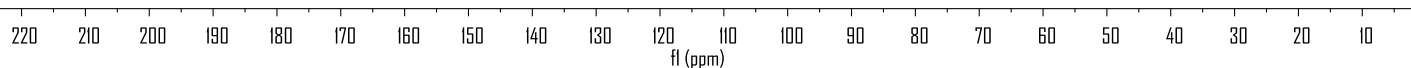


${ }^{1} \mathrm{H}$ NMR (400 MHz, $\mathrm{CDCl}_{3}$ ) spectrum of $\mathbf{3 a c}$<smiles>COc1ccccc1[C@H](NN(C(=O)[18OH])C(=O)c1ccccc1)C1CCc2ccccc2C1=O</smiles>

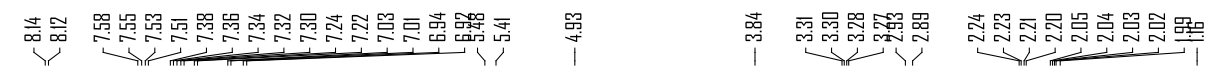

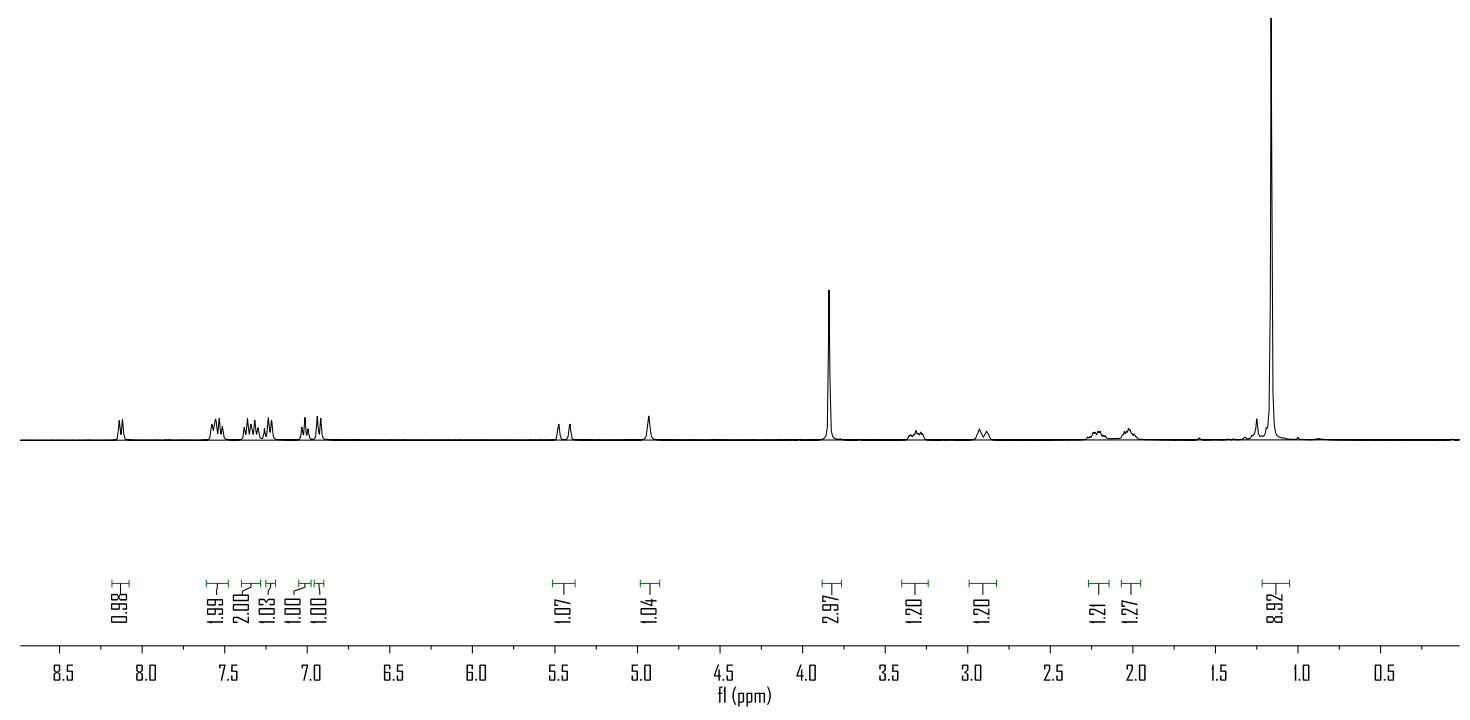


${ }^{19} \mathrm{~F}$ NMR $\left(376 \mathrm{MHz}, \mathrm{CDCl}_{3}\right.$ ) spectrum of $\mathbf{3 a c}$<smiles>COc1ccccc1[C@H](N[As]([14CH3])=O)[C@H]1CCc2ccccc2C1=O</smiles>

器哭景

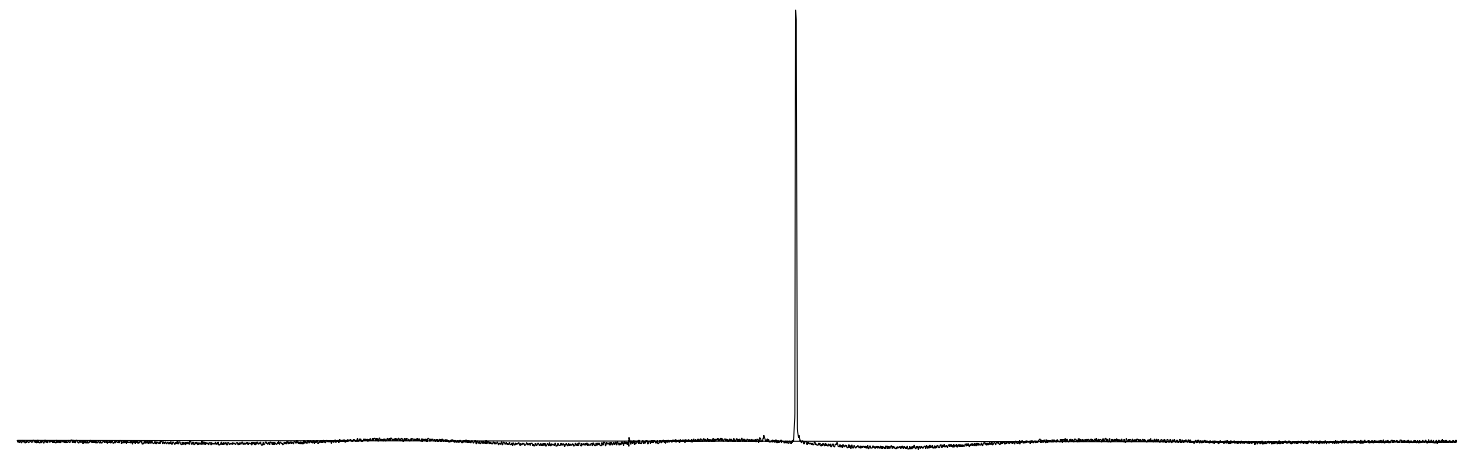

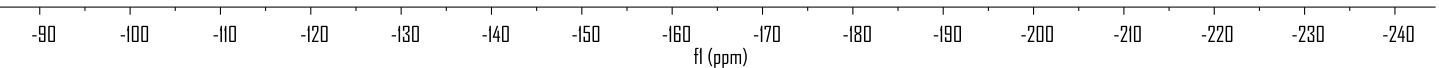


${ }^{13} \mathrm{C}$ NMR $\left(101 \mathrm{MHz}, \mathrm{CDCl}_{3}\right.$ ) spectrum of $\mathbf{3 a c}$<smiles>COc1ccccc1[C@H](N[S@]([13CH3])=O)[C@H]1CCc2ccccc2C1=O</smiles>

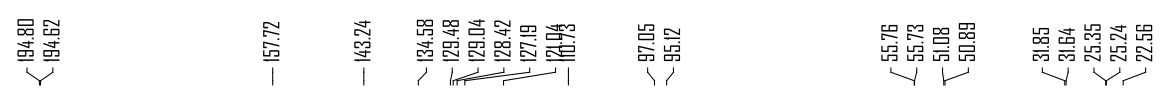

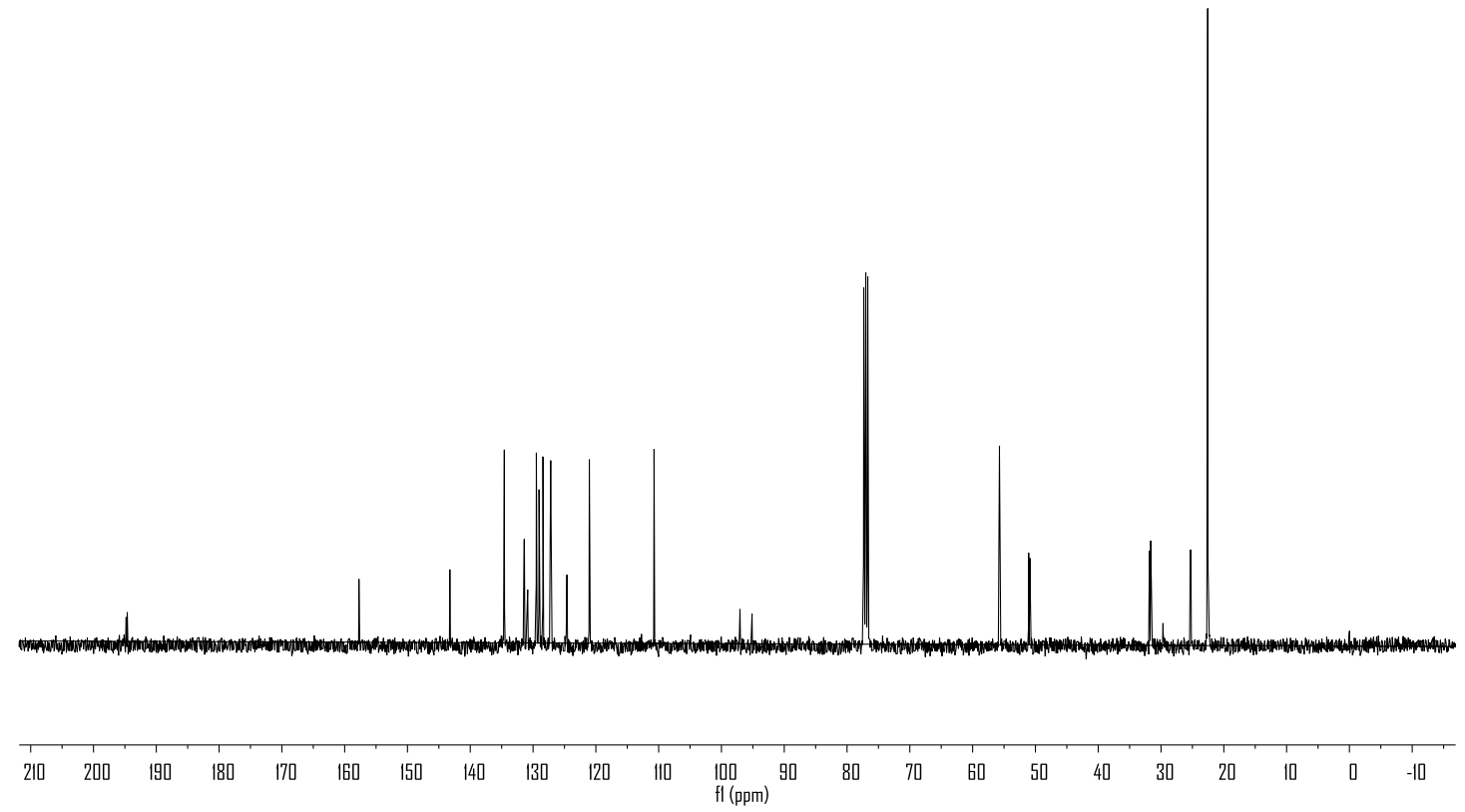


${ }^{1} \mathrm{H}$ NMR (400 MHz, $\mathrm{CDCl}_{3}$ ) spectrum of $\mathbf{3 a d}$<smiles>O=C1c2ccccc2CC[C-]1[C@H](NS(=O)[18OH])c1ccc(Cl)cc1</smiles>

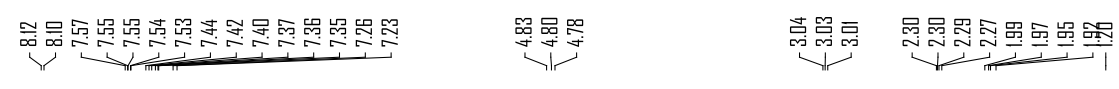

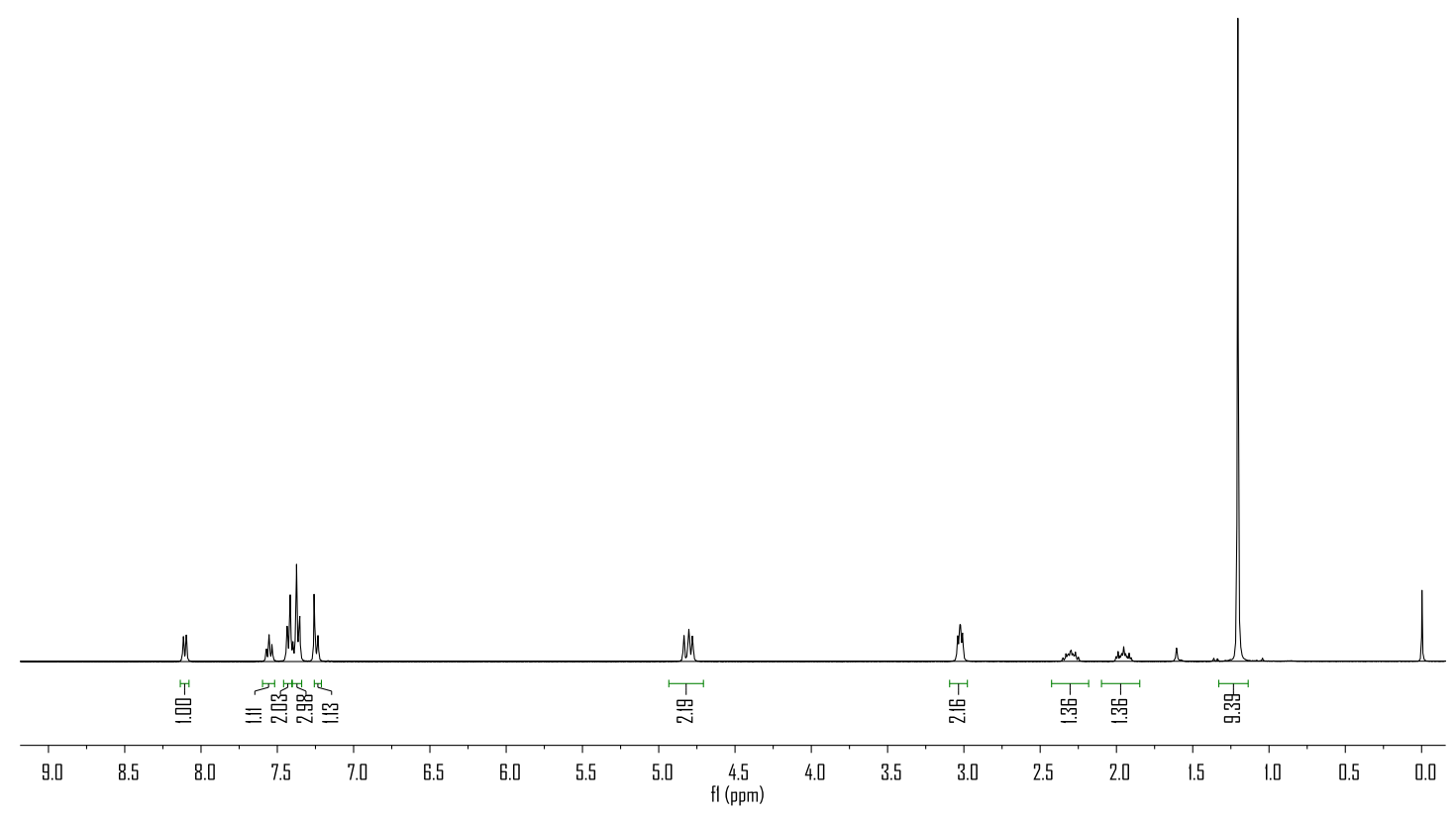


${ }^{19} \mathrm{~F}$ NMR (376 MHz, $\mathrm{CDCl}_{3}$ ) spectrum of 3ad<smiles>O=C1c2ccccc2CCC1C(NS(=O)[O-])c1ccc(Cl)cc1</smiles>

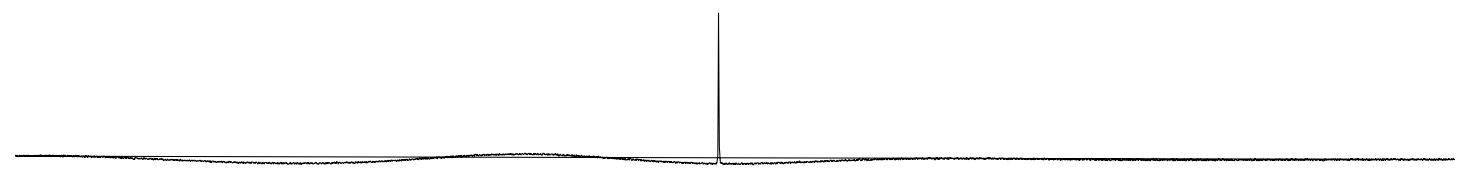

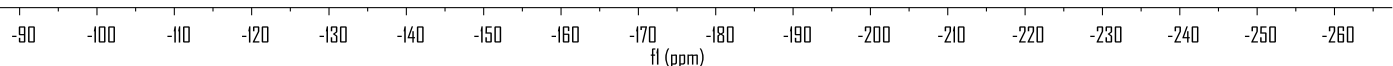


${ }^{13} \mathrm{C}$ NMR (101 MHz, $\mathrm{CDCl}_{3}$ ) spectrum of 3ad

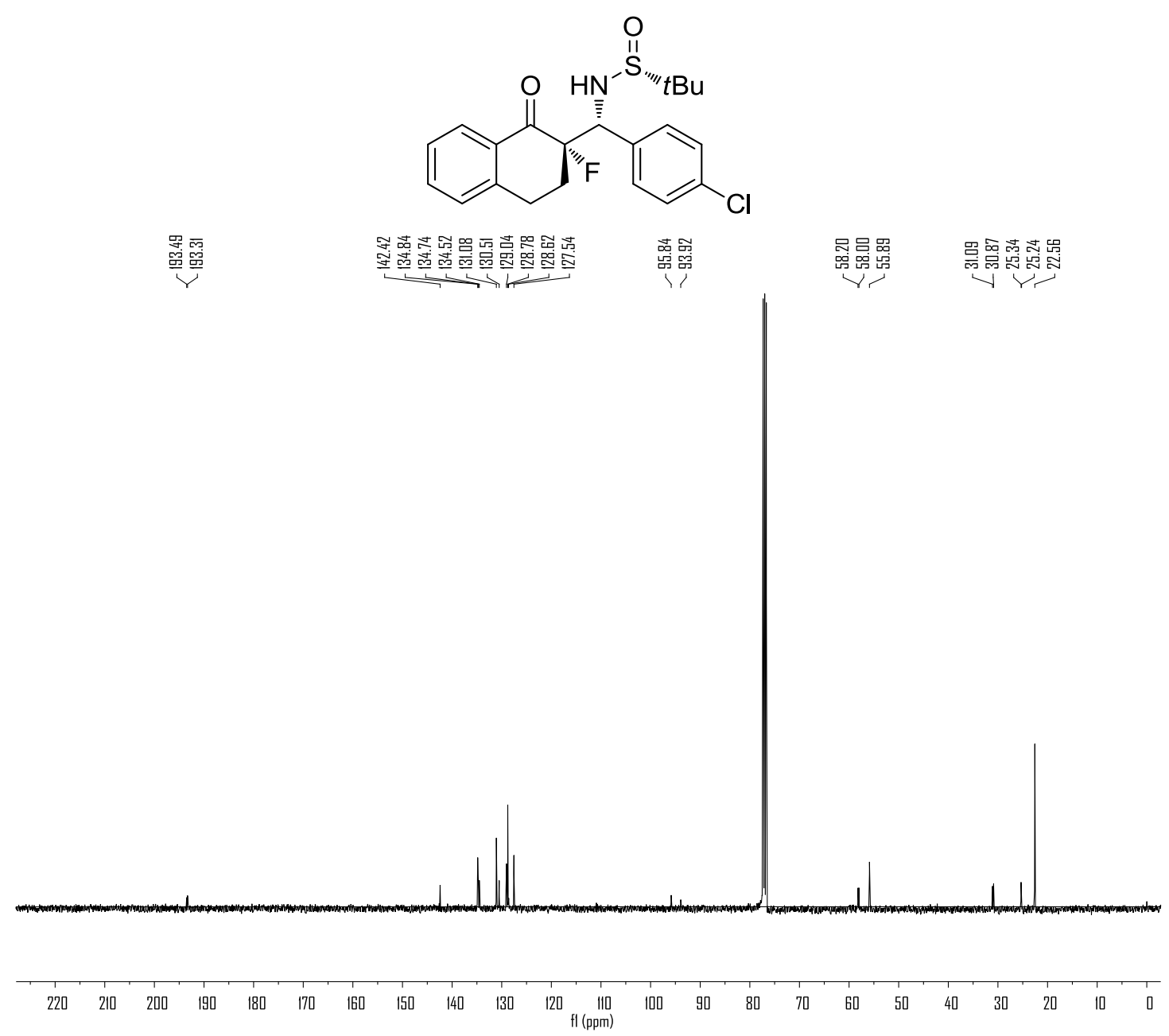


${ }^{1} \mathrm{H}$ NMR (400 MHz, $\mathrm{CDCl}_{3}$ ) spectrum of 3ae<smiles>O=C1c2ccccc2CCC1(NS(=O)[18O])c1ccco1</smiles>

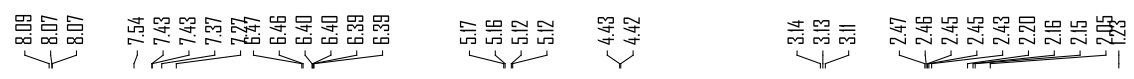

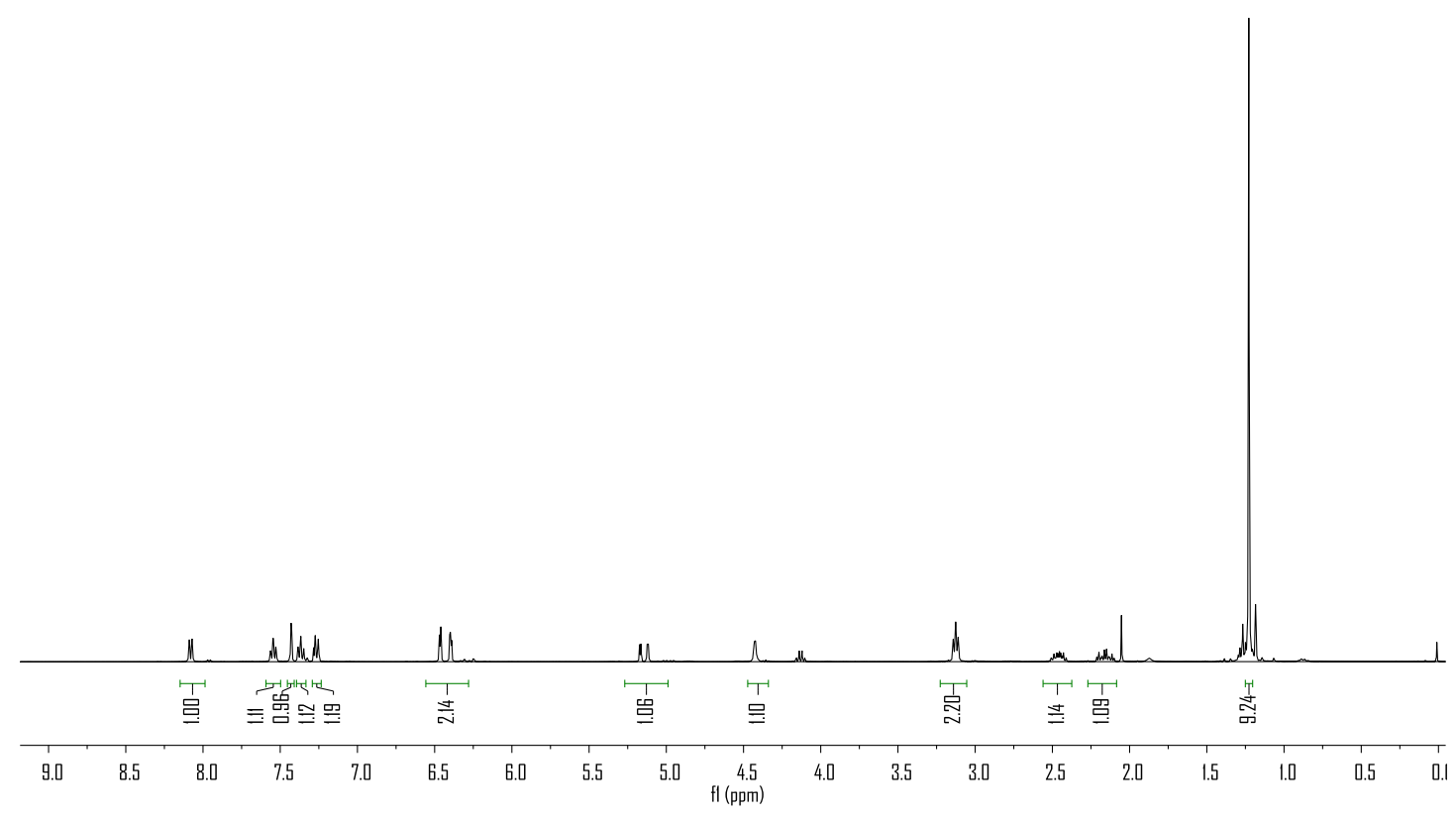


${ }^{19} \mathrm{~F} \mathrm{NMR} \mathrm{(376} \mathrm{MHz,} \mathrm{CDCl}_{3}$ ) spectrum of 3ae<smiles>O=C1c2ccccc2CC[C-]1[C@H](NS(=O)[18O])c1ccco1</smiles>

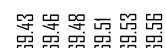

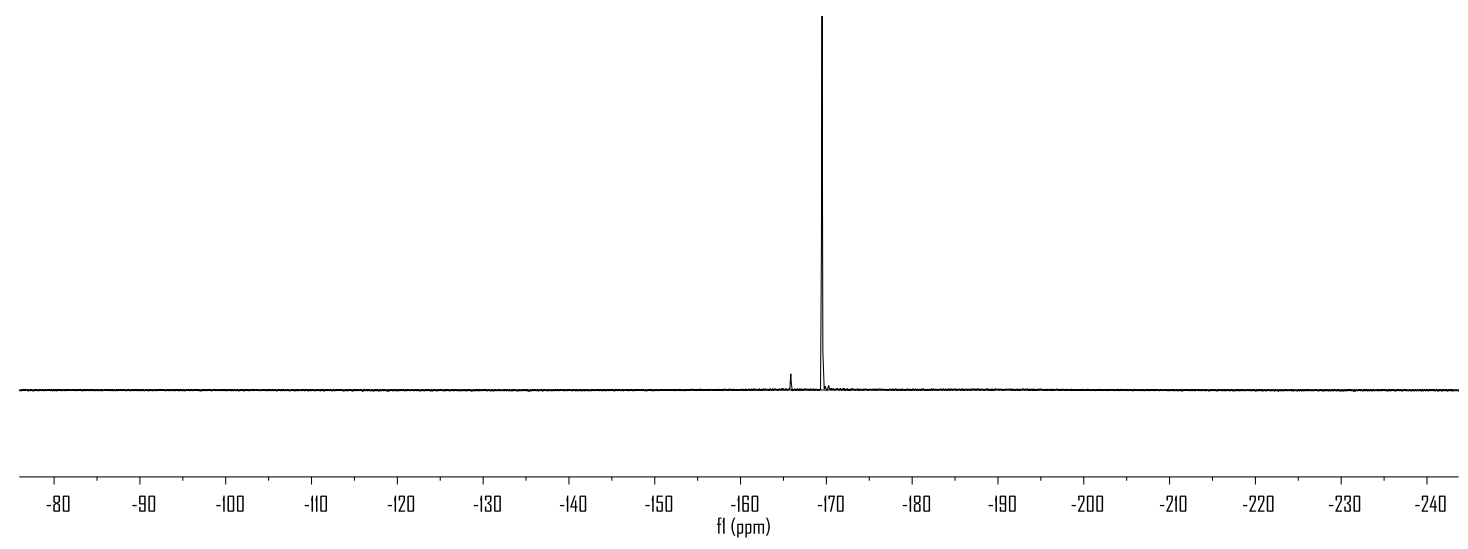


${ }^{13} \mathrm{C}$ NMR $\left(101 \mathrm{MHz}, \mathrm{CDCl}_{3}\right)$ spectrum of 3ae<smiles>CC(C)(C)S(=O)N[C@H](c1ccco1)C1(F)CCc2ccccc2C1=O</smiles>

怘歌

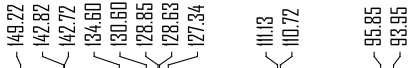

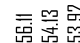

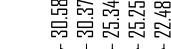

$\checkmark r$

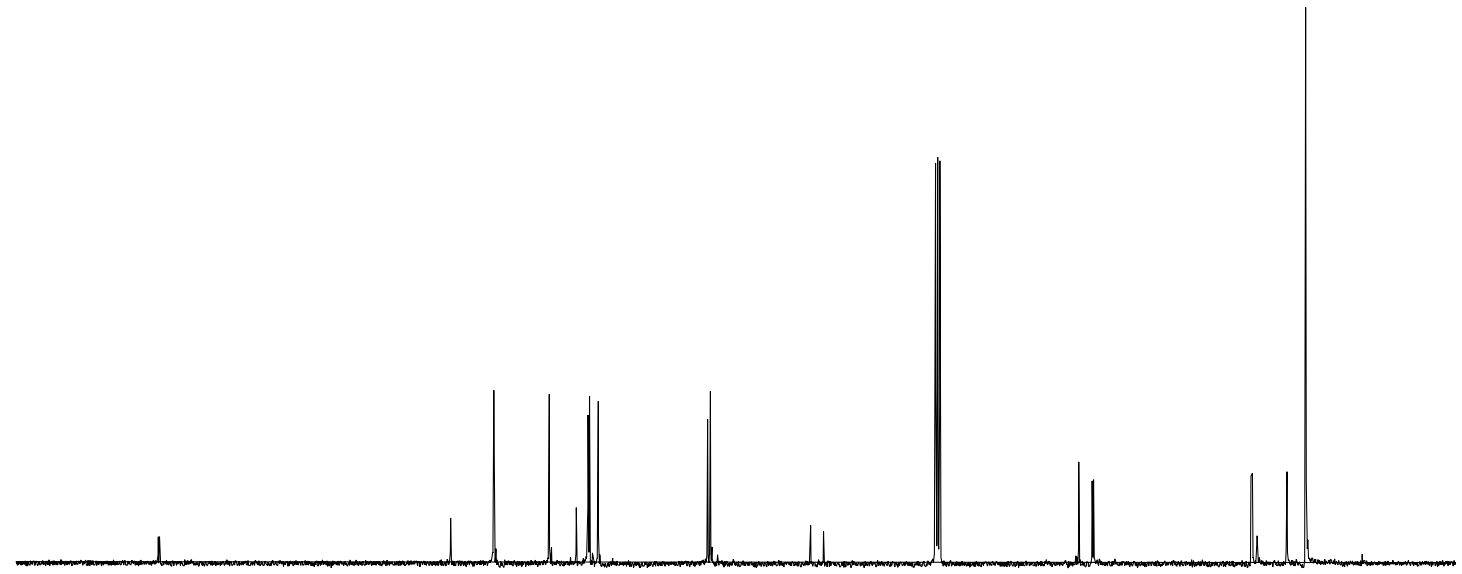

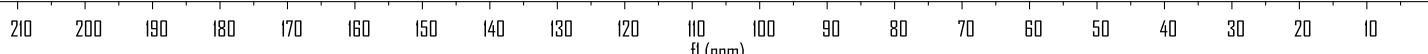


${ }^{1} \mathrm{H}$ NMR $\left(400 \mathrm{MHz}, \mathrm{CDCl}_{3}\right)$ spectrum of $3 \mathbf{a f}$<smiles>CC1([C@H](/C=C/c2ccccc2)NS(=O)/C=C\c2ccccc2)CCc2ccccc2C1=O</smiles>

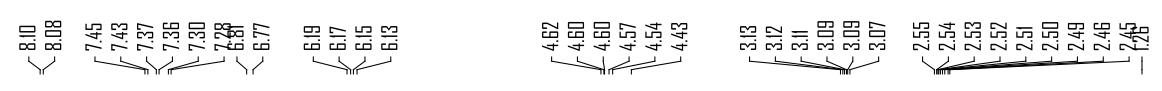

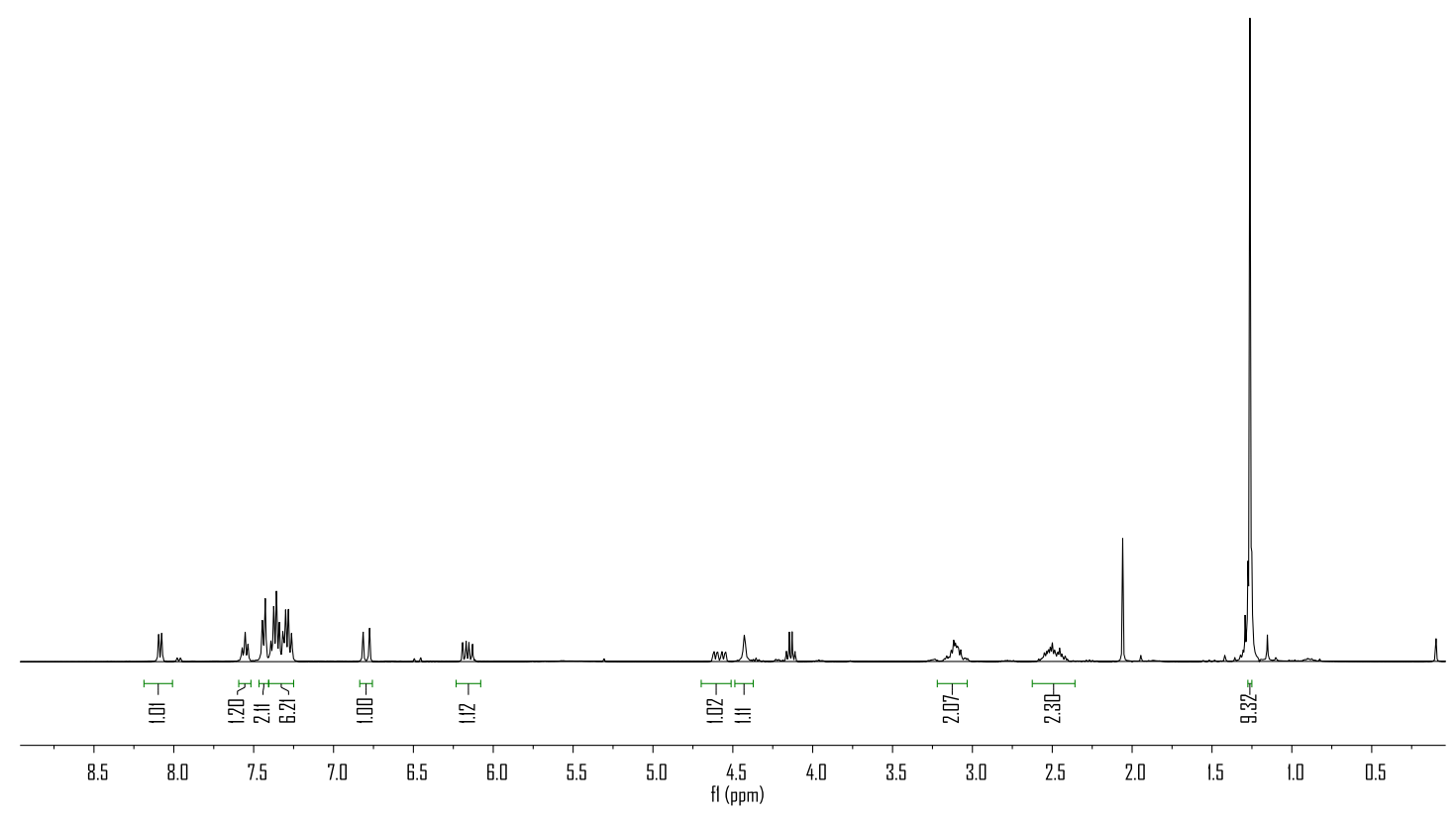


${ }^{19} \mathrm{~F}$ NMR $\left(376 \mathrm{MHz}, \mathrm{CDCl}_{3}\right.$ ) spectrum of 3af<smiles>CC(C)(C)S(=O)NC(/C=C/c1ccccc1)C1(F)CCc2ccccc2C1=O</smiles>

i

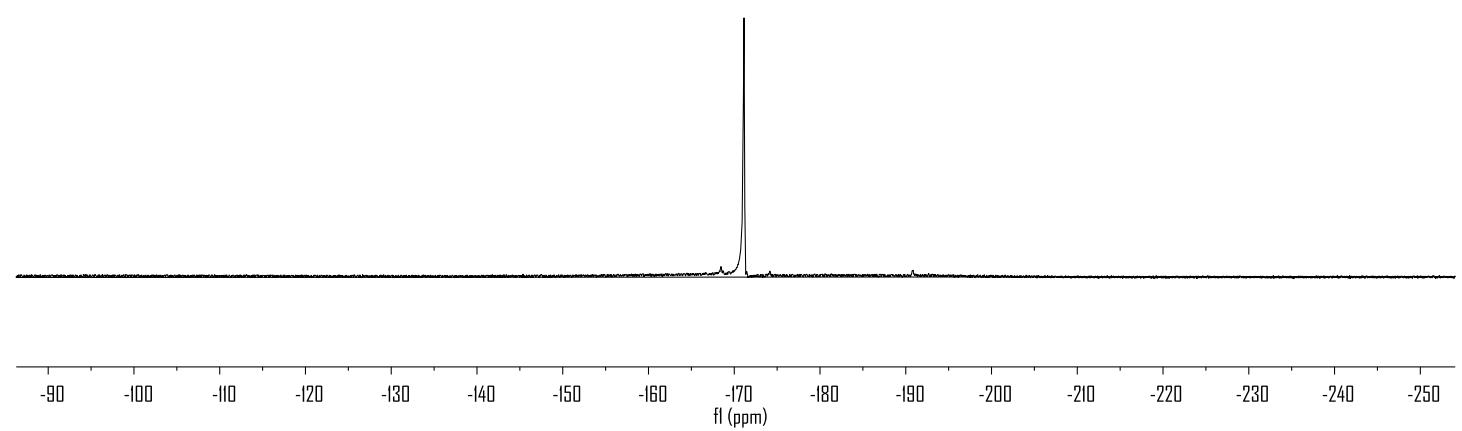


${ }^{13} \mathrm{C}$ NMR $\left(101 \mathrm{MHz}, \mathrm{CDCl}_{3}\right.$ ) spectrum of 3af<smiles>CC(=O)N[C@H](/C=C/c1ccccc1)C1CCc2ccccc2C1=O</smiles>

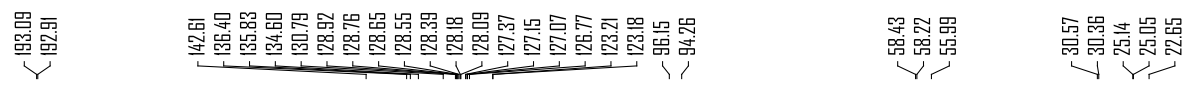

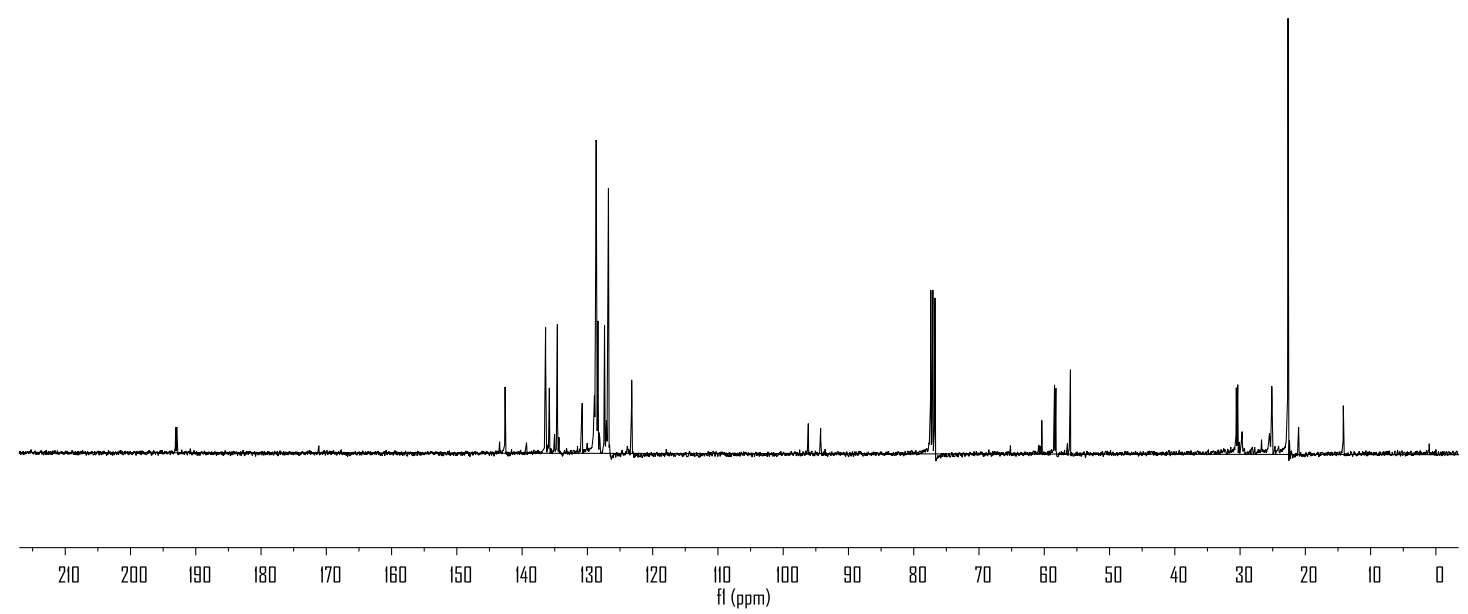


${ }^{1} \mathrm{H}$ NMR (400 MHz, $\mathrm{CDCl}_{3}$ ) spectrum of $\mathbf{3 b g}$<smiles>CCC[C@H](NC(=O)[18O])C1COc2ccccc2C1=O</smiles>

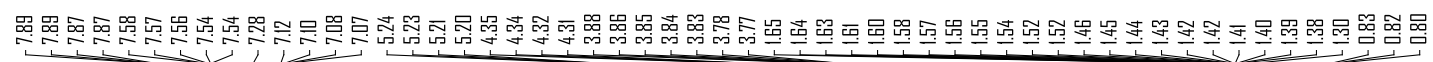

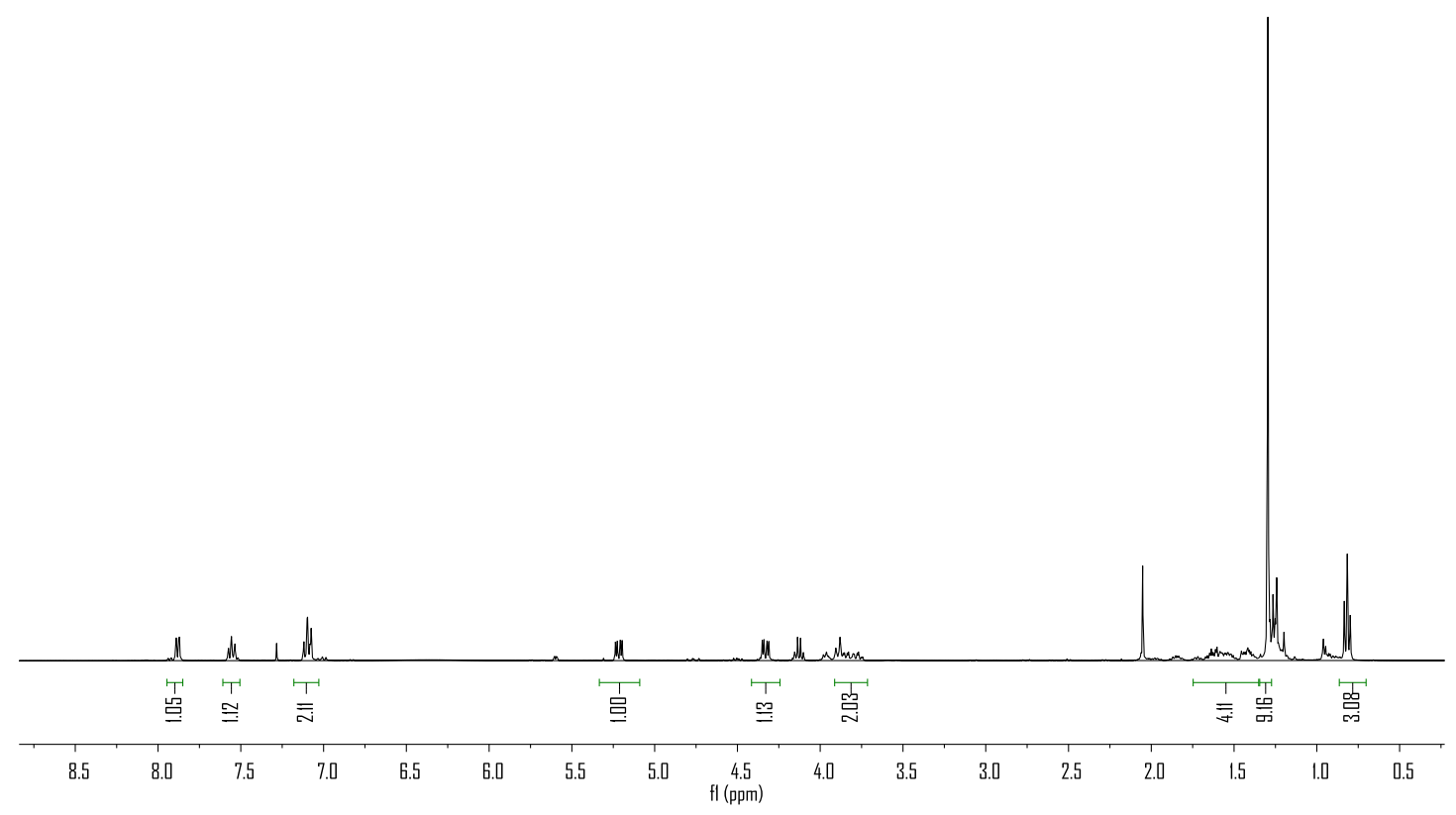


${ }^{19} \mathrm{~F}$ NMR (376 MHz, $\mathrm{CDCl}_{3}$ ) spectrum of $\mathbf{3 b g}$<smiles>CCC[C@H](NS(=O)CCC)C1COc2ccccc2C1=O</smiles>

器器

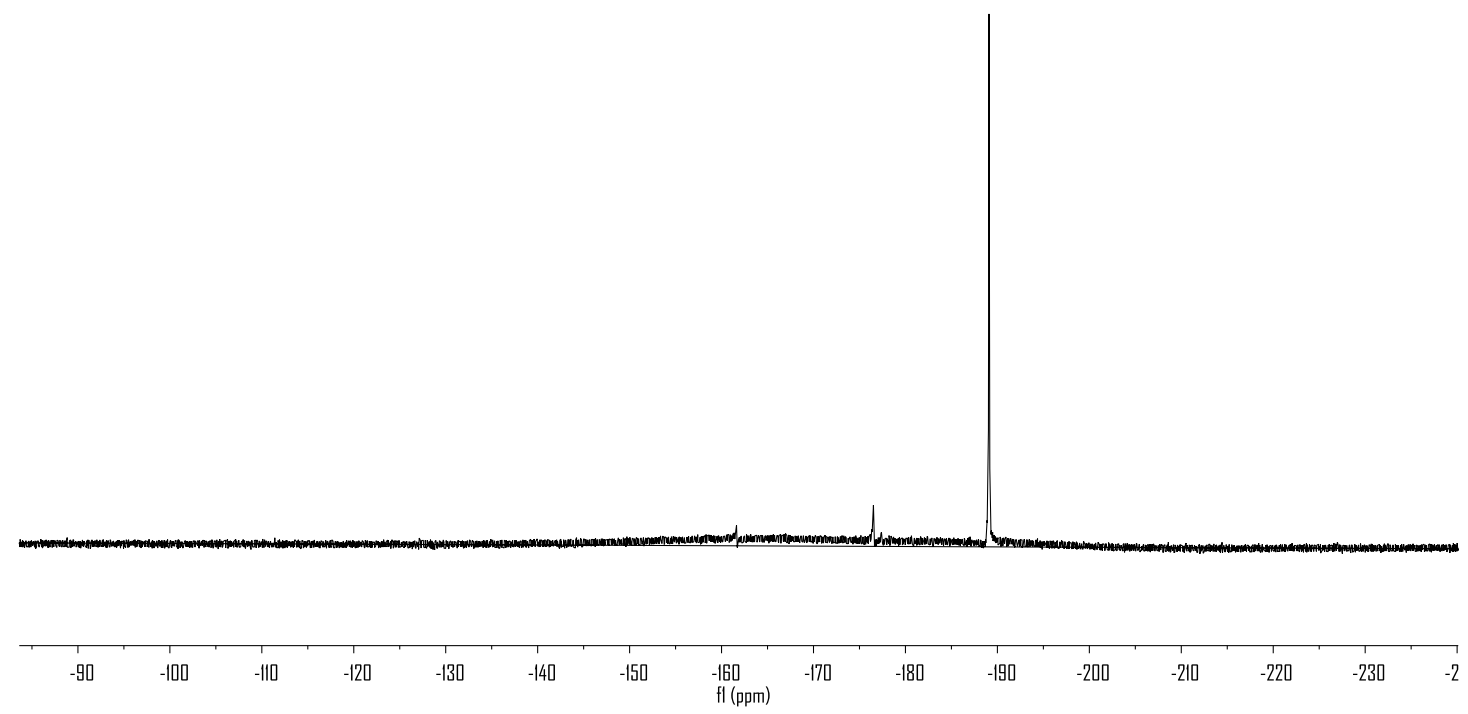


${ }^{13} \mathrm{C}$ NMR (101 MHz, $\mathrm{CDCl}_{3}$ ) spectrum of $\mathbf{3 b g}$

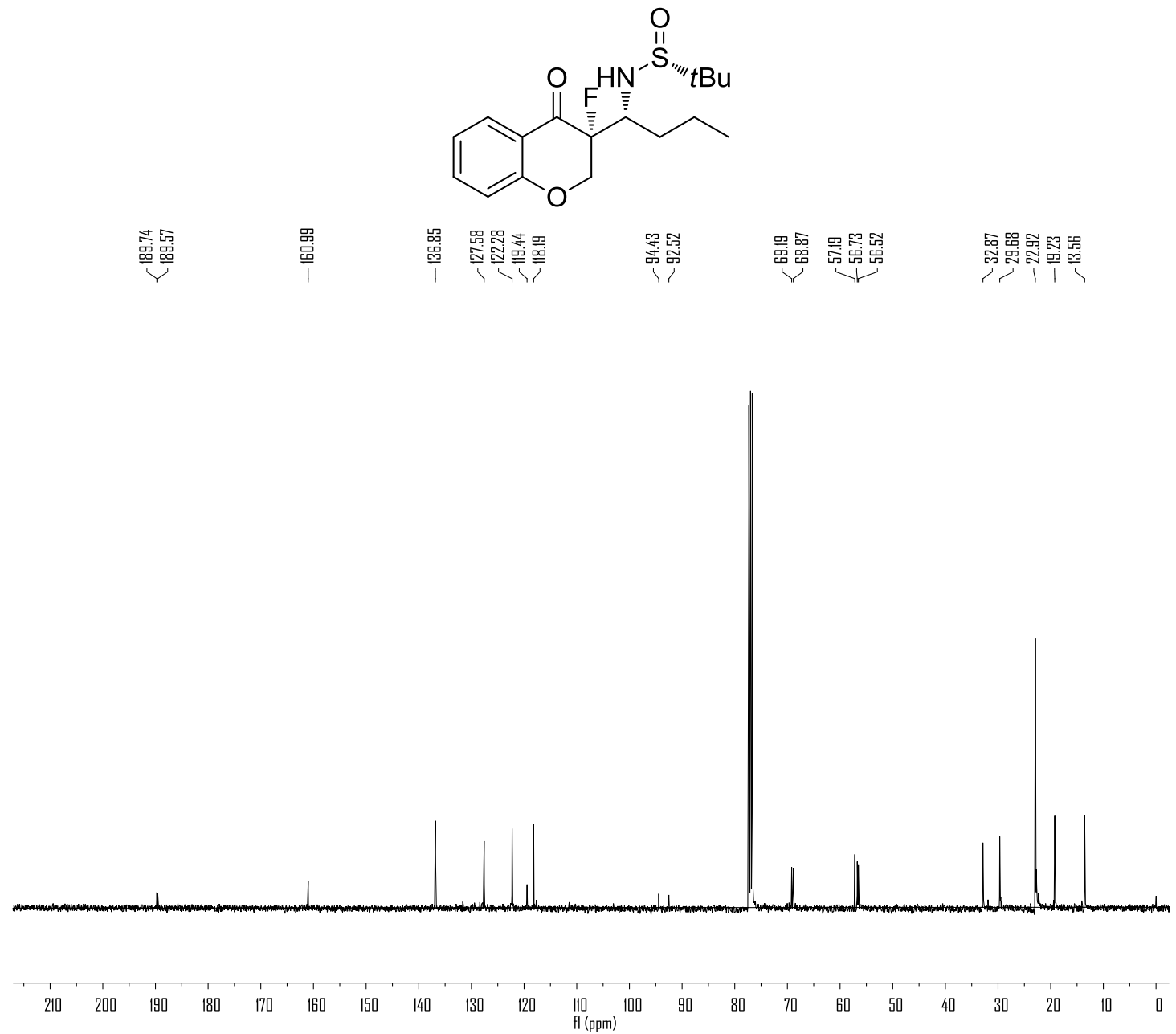


${ }^{1} \mathrm{H}$ NMR (400 MHz, $\mathrm{CDCl}_{3}$ ) spectrum of $\mathbf{3 b h}$<smiles>CCCC[C@H](NC(=O)[18O])C1COc2ccccc2C1=O</smiles>

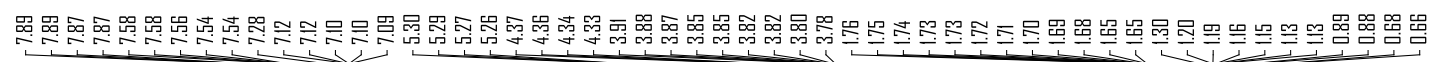

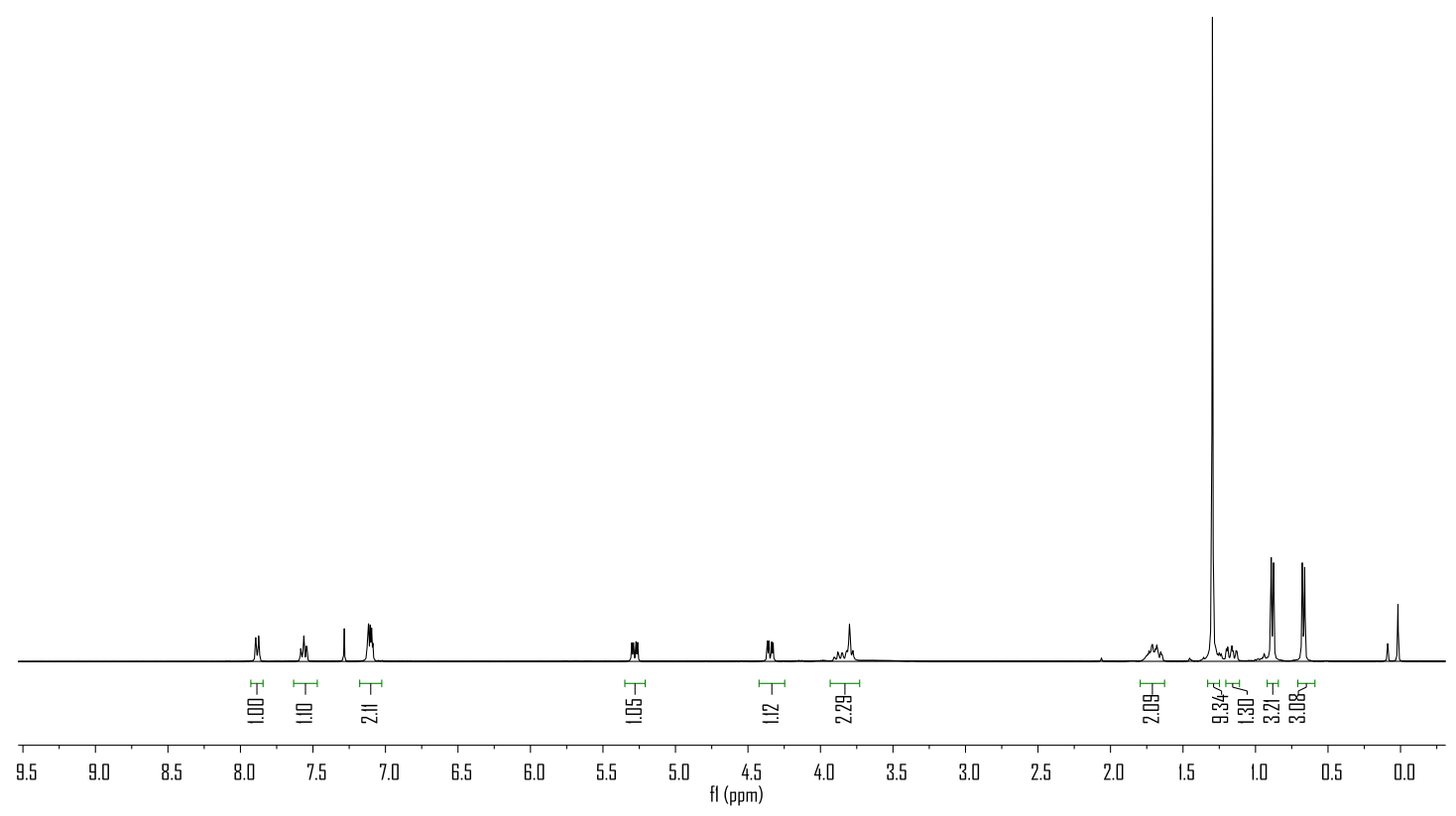


${ }^{19} \mathrm{~F}$ NMR (376 MHz, $\mathrm{CDCl}_{3}$ ) spectrum of $\mathbf{3 b h}$<smiles>CC(C)(C)CC[C@H](NC(=O)[18O])C1COc2ccccc2C1=O</smiles>

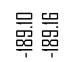

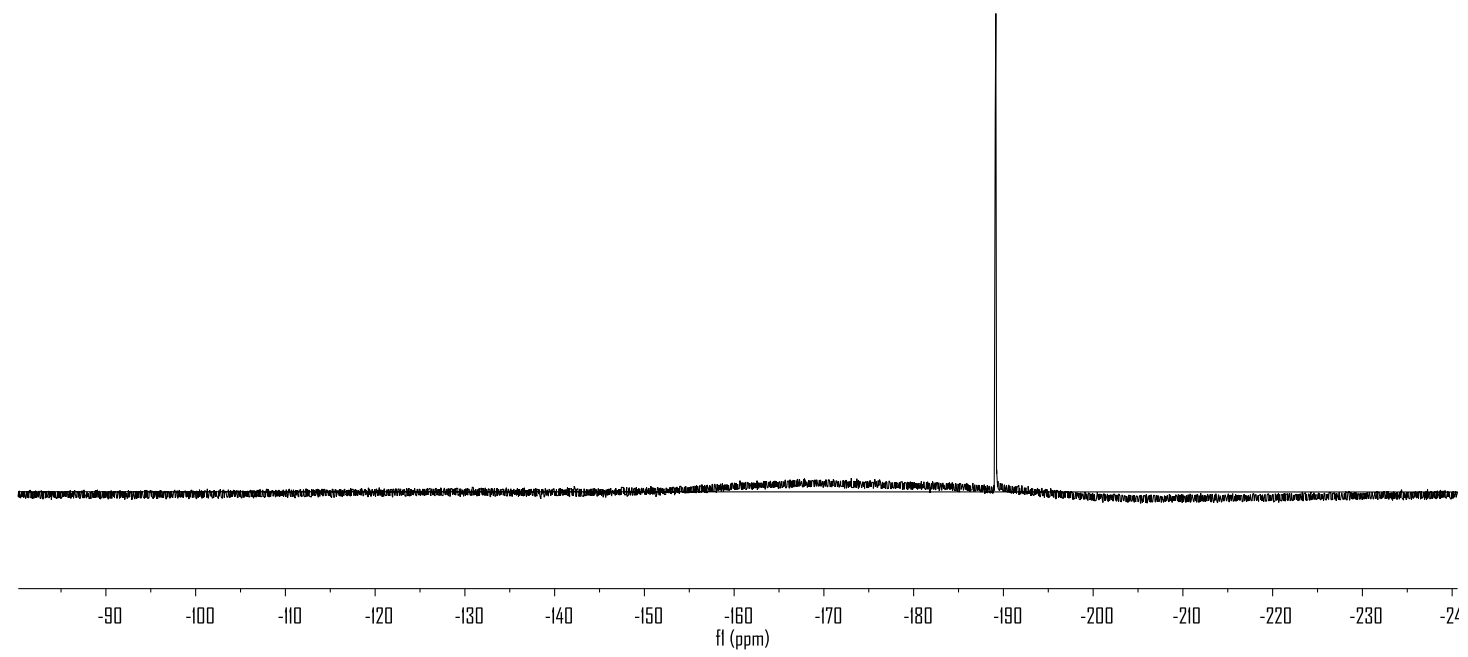


${ }^{13} \mathrm{C}$ NMR (101 MHz, $\mathrm{CDCl}_{3}$ ) spectrum of $\mathbf{3 b h}$

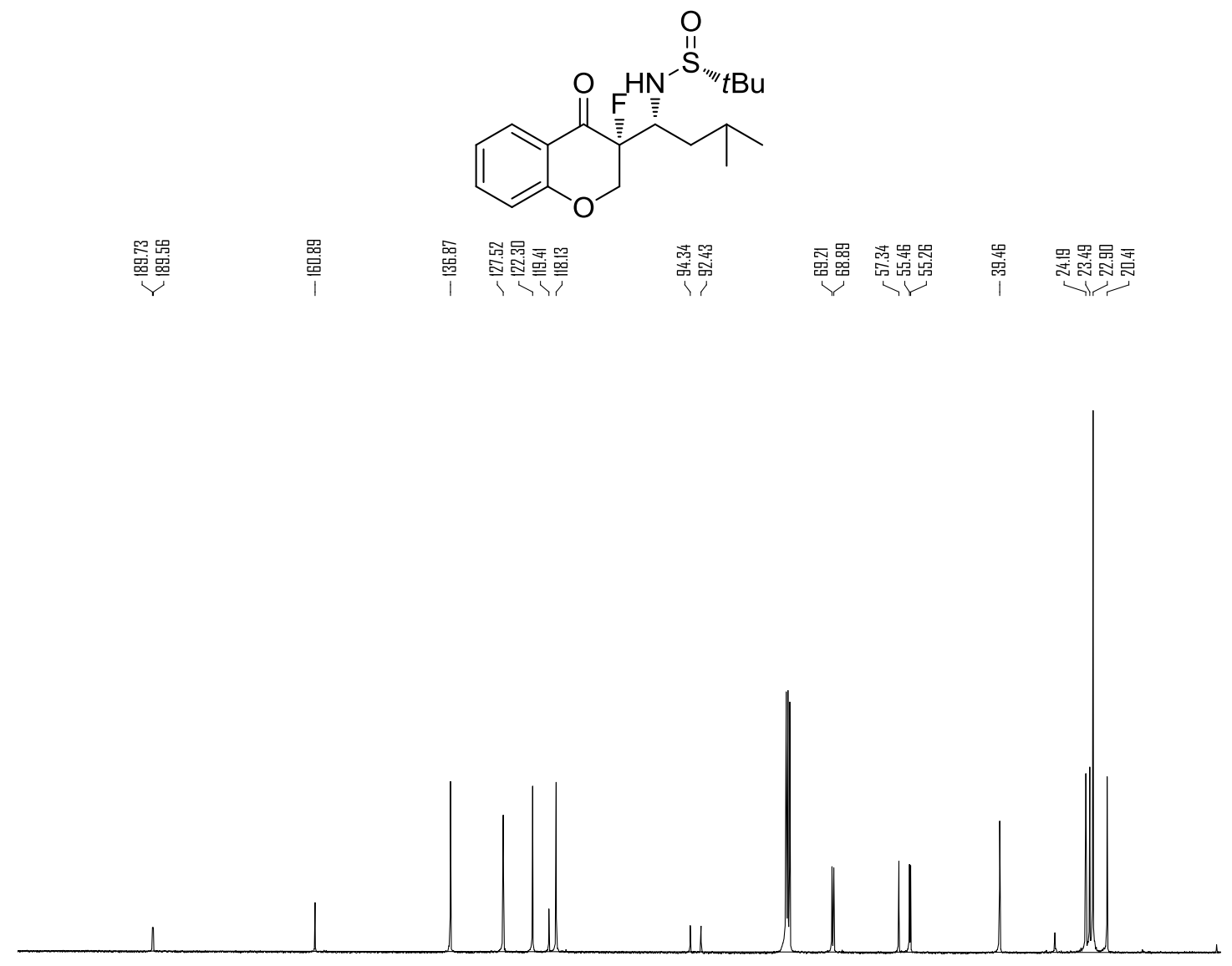

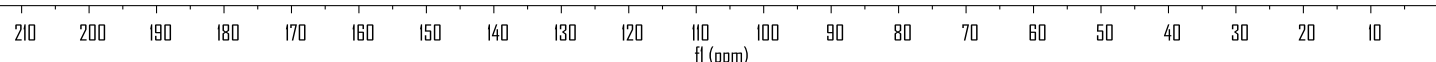


${ }^{1} \mathrm{H}$ NMR (400 MHz, $\mathrm{CDCl}_{3}$ ) spectrum of $\mathbf{3 d b}$<smiles>CCC(C)S(=O)N[C@H](c1ccc(C)cc1)C1CCCc2ccccc2C1=O</smiles>

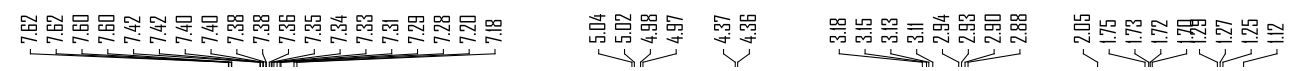

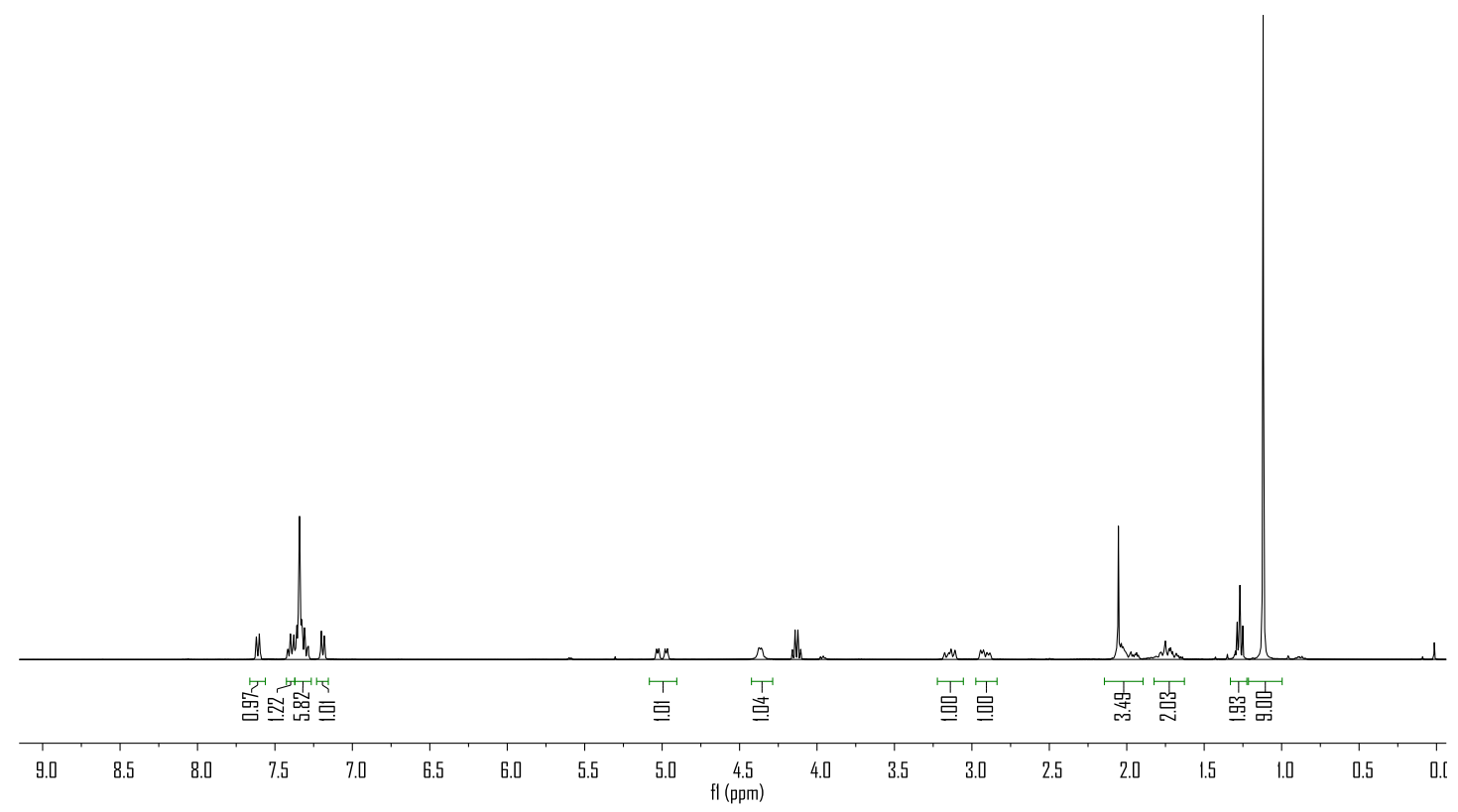


${ }^{19} \mathrm{~F}$ NMR (376 MHz, $\mathrm{CDCl}_{3}$ ) spectrum of $\mathbf{3 d b}$<smiles>CC(=O)N[C@@H](C1=CCCc2ccccc2C1=O)c1ccc(C)cc1</smiles> 
${ }^{13} \mathrm{C}$ NMR (101 MHz, $\mathrm{CDCl}_{3}$ ) spectrum of $\mathbf{3 d b}$

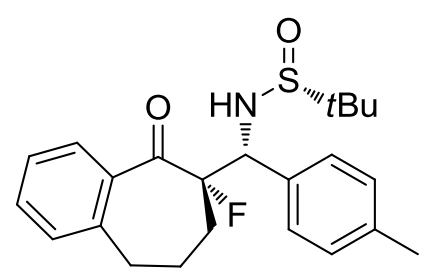

嘉㷼

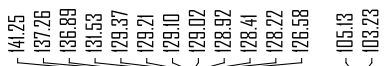

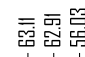

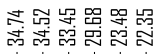

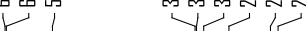
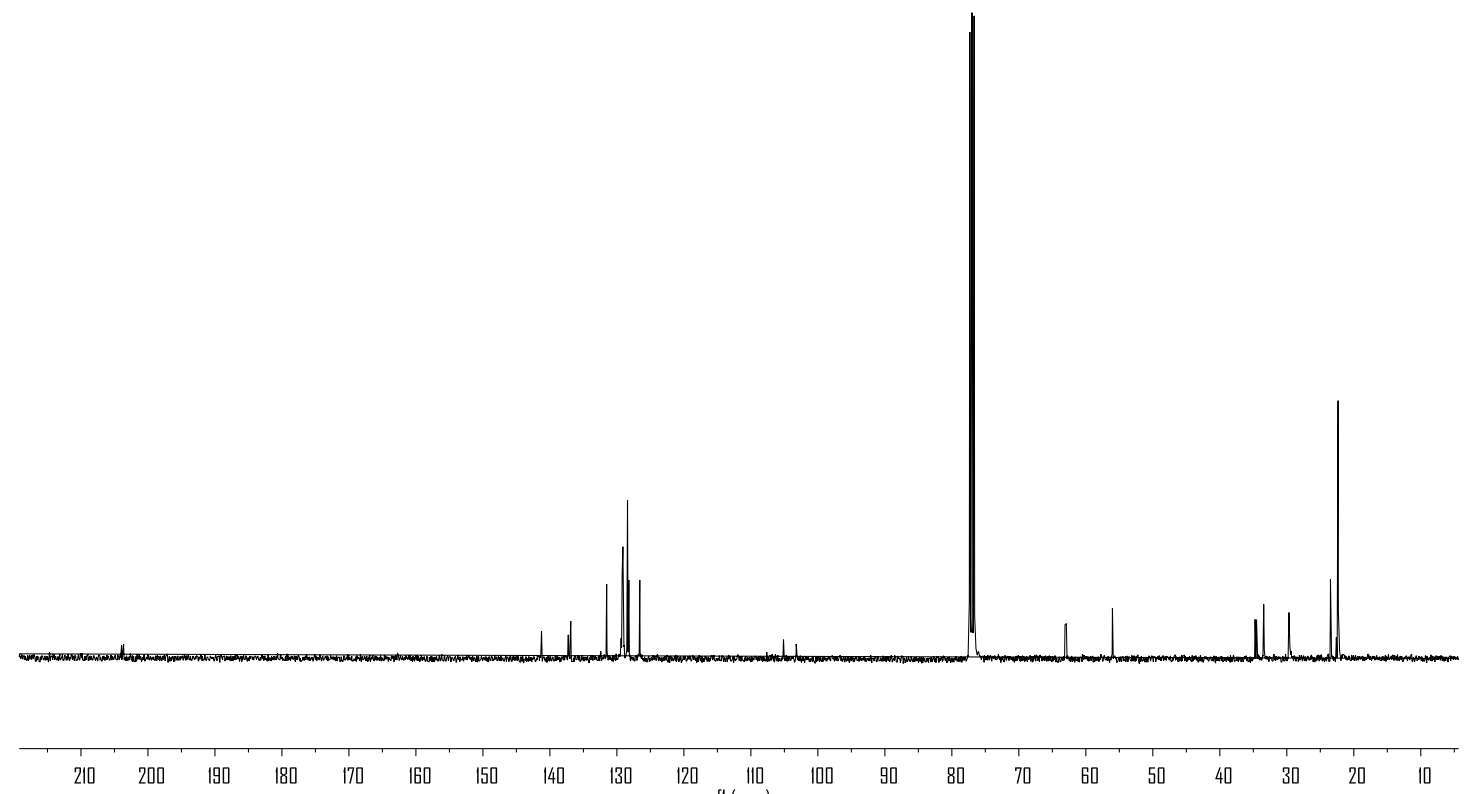

$150 \quad 14$

$\begin{array}{lll}130 & 120 & \mathrm{IID} \\ \mathrm{fl}(\mathrm{ppm}) & \end{array}$

$100 \quad 90$

70

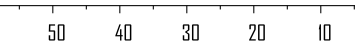


${ }^{1} \mathrm{H}$ NMR (400 MHz, $\mathrm{CDCl}_{3}$ ) spectrum of $\mathbf{3 g d}$<smiles>CC(=O)N[C@H](c1ccc(Cl)cc1)C(C)(C)C(=O)c1ccccc1</smiles>

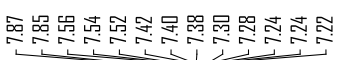

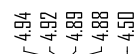

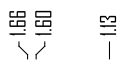

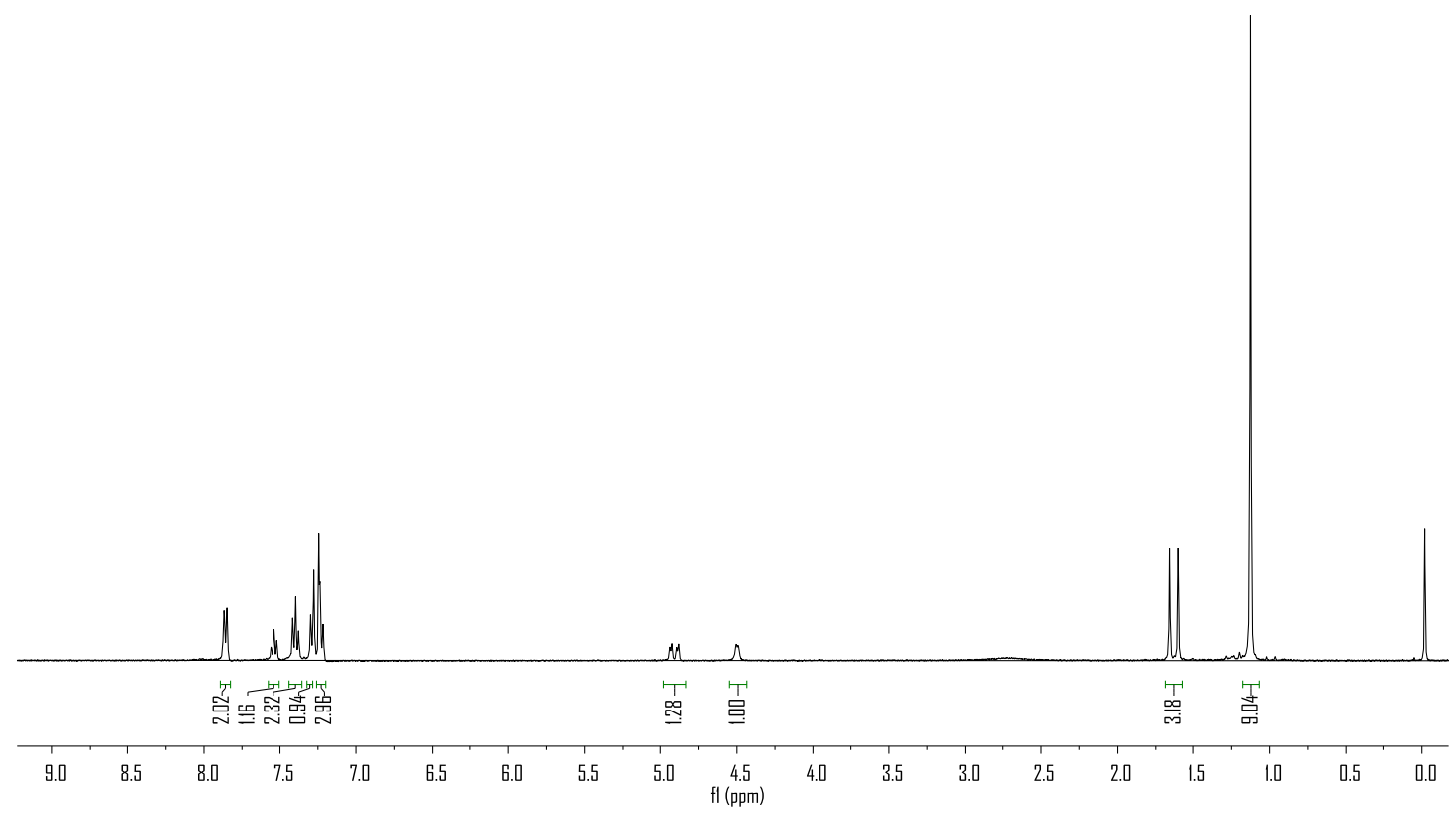


${ }^{19} \mathrm{~F} \mathrm{NMR}\left(376 \mathrm{MHz}, \mathrm{CDCl}_{3}\right)$ spectrum of $\mathbf{3 g d}$<smiles>CC(F)(C(=O)c1ccccc1)[C@H](NS(=O)C(C)(C)C)c1ccc(Cl)cc1</smiles>

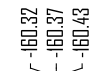

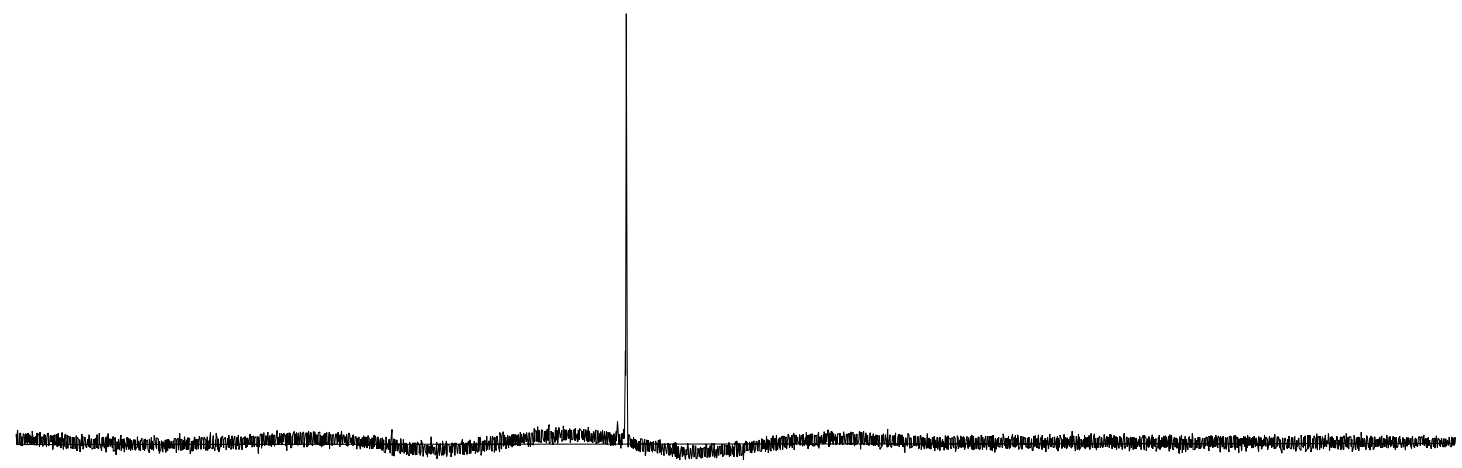

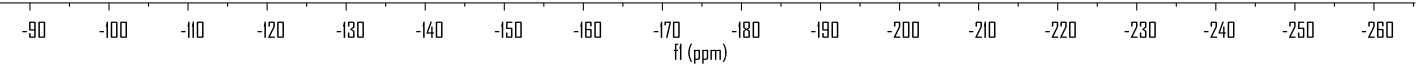


${ }^{13} \mathrm{C}$ NMR (101 MHz, $\mathrm{CDCl}_{3}$ ) spectrum of $\mathbf{3 g d}$<smiles>CC(F)(C(=O)c1ccccc1)[C@H](NS(=O)C(C)(C)C)c1ccc(Cl)cc1</smiles>

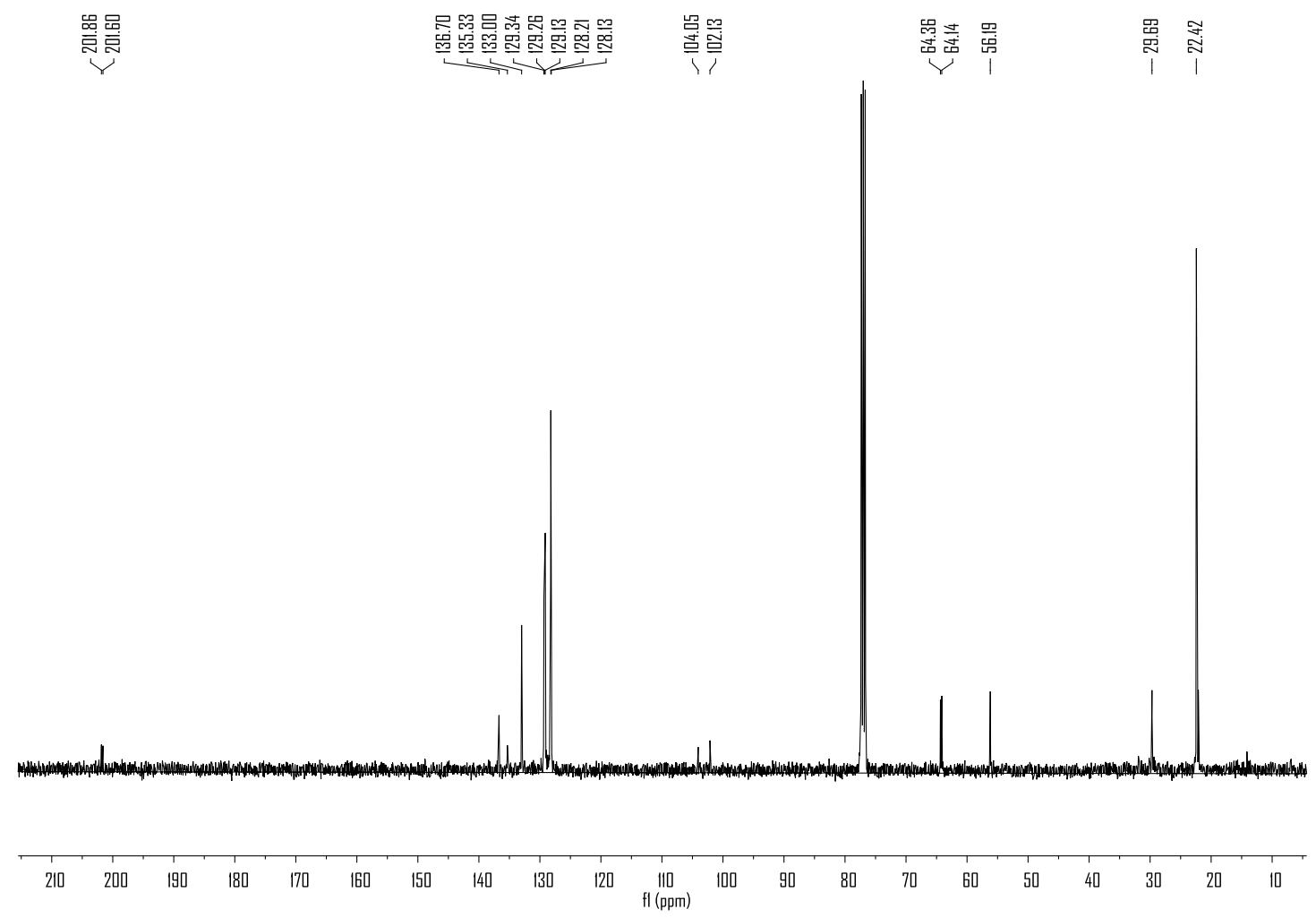


${ }^{1} \mathrm{H}$ NMR $\left(400 \mathrm{MHz}, \mathrm{CDCl}_{3}\right)$ spectrum of $\mathbf{3 h b}$<smiles>Cc1ccc(C(N[S@](=O)[O-])C(F)C(=O)C23CC4CC(CC(C4)C2)C3)cc1</smiles>

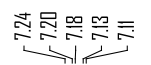

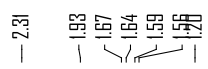

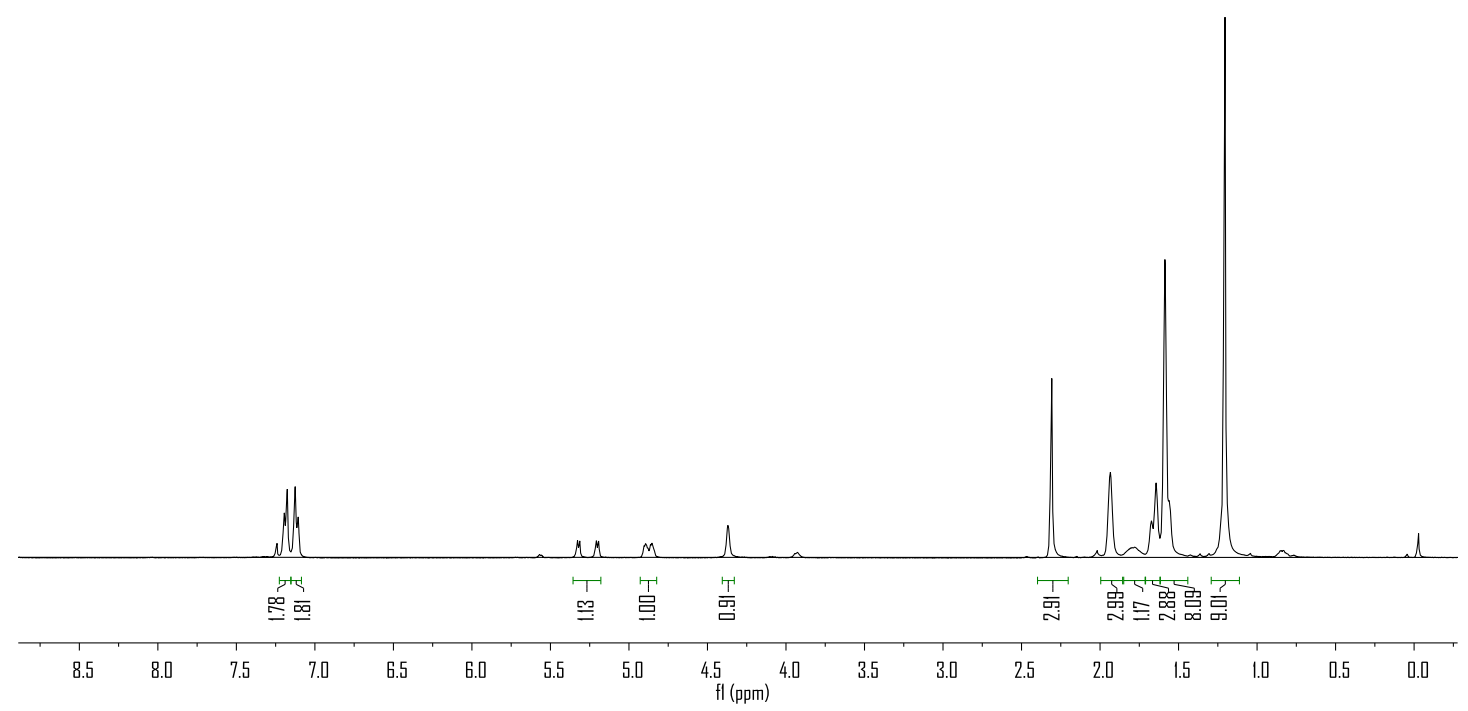


${ }^{19} \mathrm{~F}$ NMR (376 MHz, $\mathrm{CDCl}_{3}$ ) spectrum of $\mathbf{3 h b}$<smiles>Cc1ccc([C@H](NN[S@@](=O)[O-])[C@H](F)C(=O)C23CC4CC(CC(C4)C2)C3)cc1</smiles>

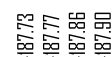

ij

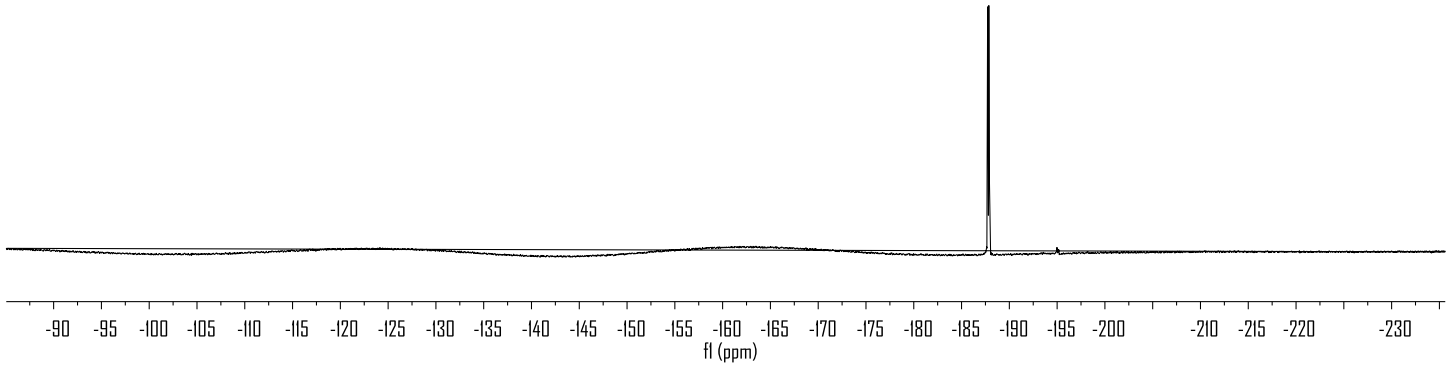


${ }^{13} \mathrm{C}$ NMR $\left(101 \mathrm{MHz}, \mathrm{CDCl}_{3}\right.$ ) spectrum of $\mathbf{3 h b}$<smiles>Cc1ccc(C(N[R5](=O)[O-])C(F)C(=O)C23CC4CC(CC(C4)C2)C3)cc1</smiles>

总品

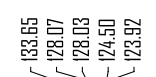

옹몽

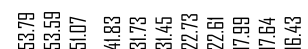

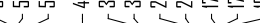

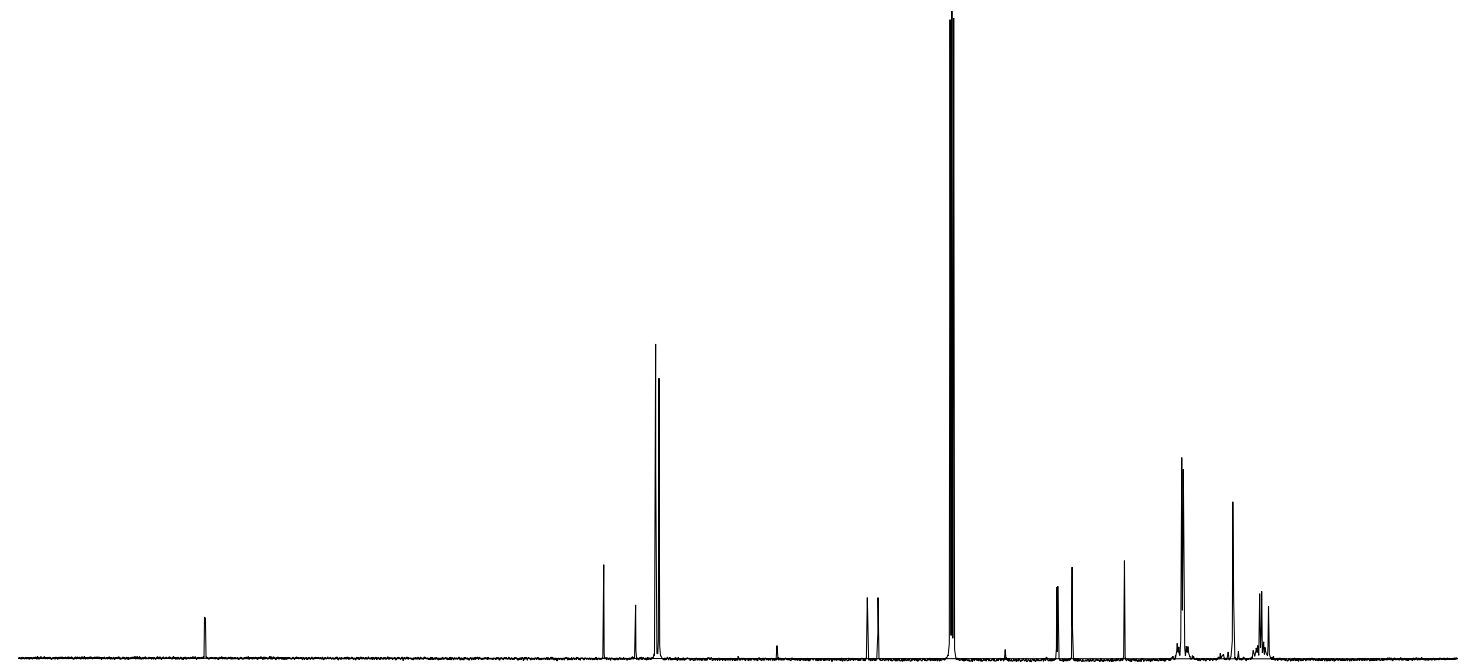

$\begin{array}{llllllllllllllllllllllllll}230 & 220 & 210 & 200 & 190 & 180 & 170 & 160 & 150 & 140 & 130 & 120 & 1110 & 100 & 90 & 80 & 70 & 60 & 50 & 40 & 30 & 20 & 10 & 0 & -10\end{array}$ 
${ }^{1} \mathrm{H}$ NMR (400 MHz, $\mathrm{CDCl}_{3}$ ) spectrum of 3 hi

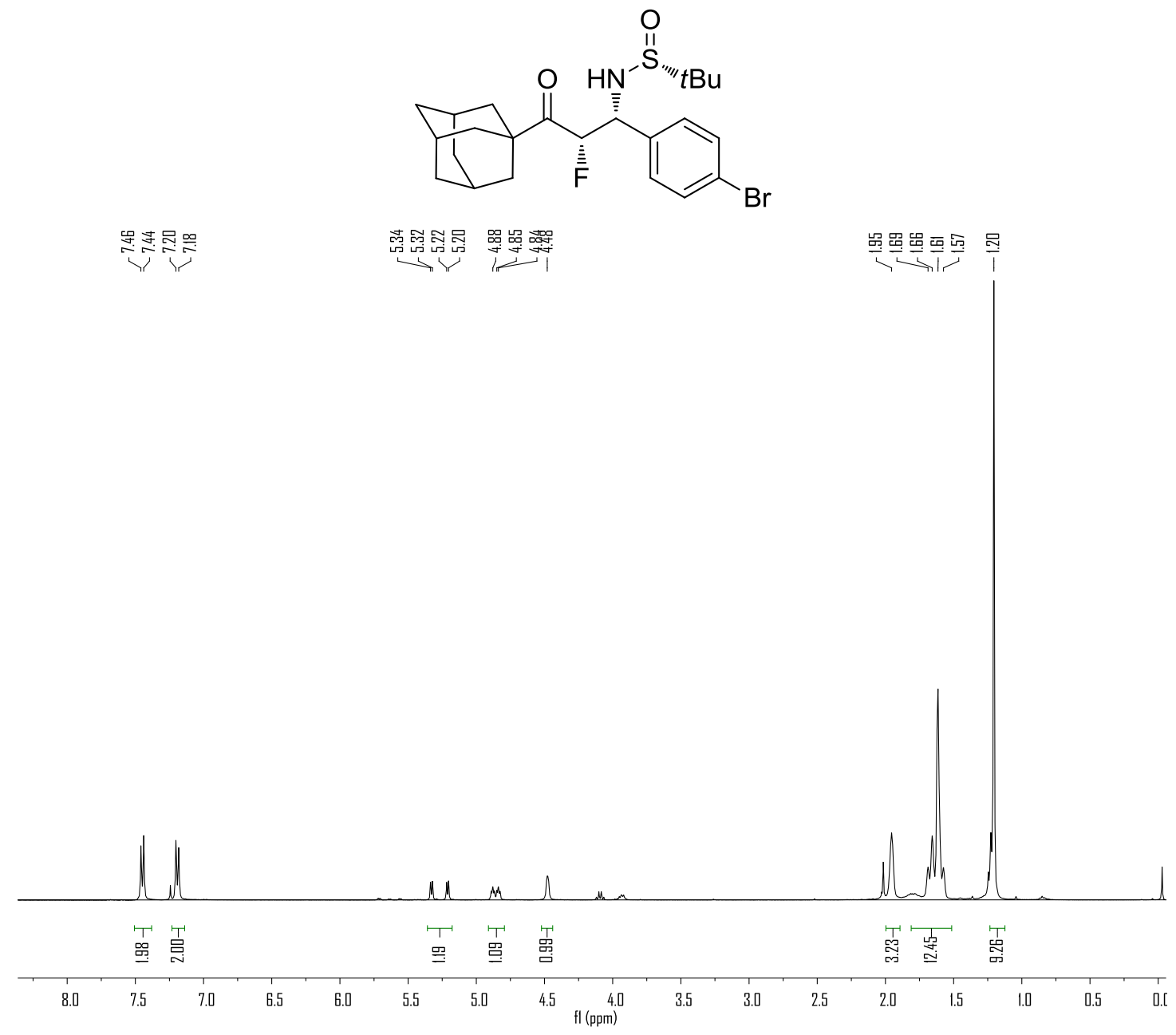


${ }^{19} \mathrm{~F}$ NMR (376 MHz, $\mathrm{CDCl}_{3}$ ) spectrum of $\mathbf{3 h i}$<smiles>CC(C)(C)[13C](=O)N[C@H](c1ccc(Br)cc1)C(F)C(=O)C12CC3CC(CC(C3)C1)C2</smiles>

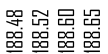

这

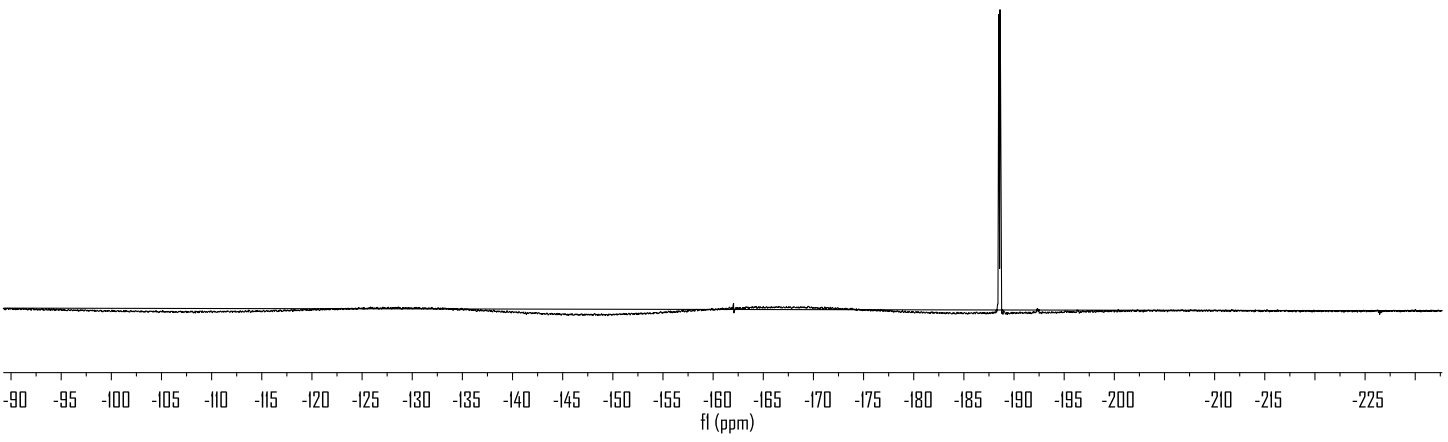


${ }^{13} \mathrm{C}$ NMR (101 MHz, $\mathrm{CDCl}_{3}$ ) spectrum of $\mathbf{3 h i}$<smiles>CC(=O)N[C@H](c1ccc(Br)cc1)C(F)C(=O)C12CC3CC(CC(C3)C1)C2</smiles>

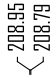

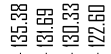

言慰

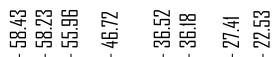

1)

\1

X) ।

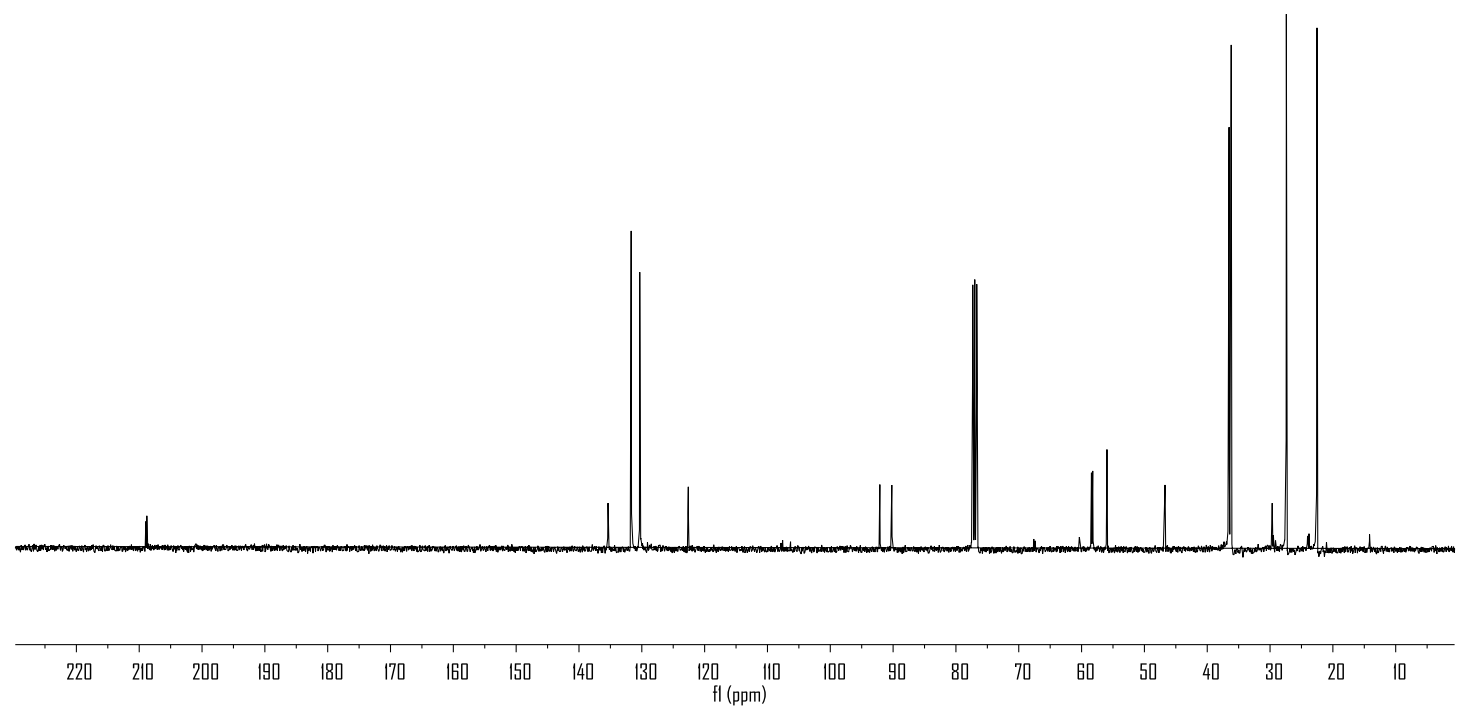


${ }^{1} \mathrm{H}$ NMR (400 MHz, $\mathrm{CDCl}_{3}$ ) spectrum of $\mathbf{3 h j}$<smiles>CCC(C)(C)S(=O)N[C@H](c1ccc(C(=O)OC)cc1)[C@H](F)C(=O)C12CC3CC(CC(C3)C1)C2</smiles>

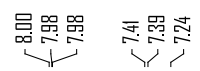

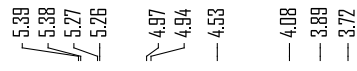

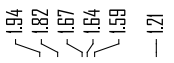

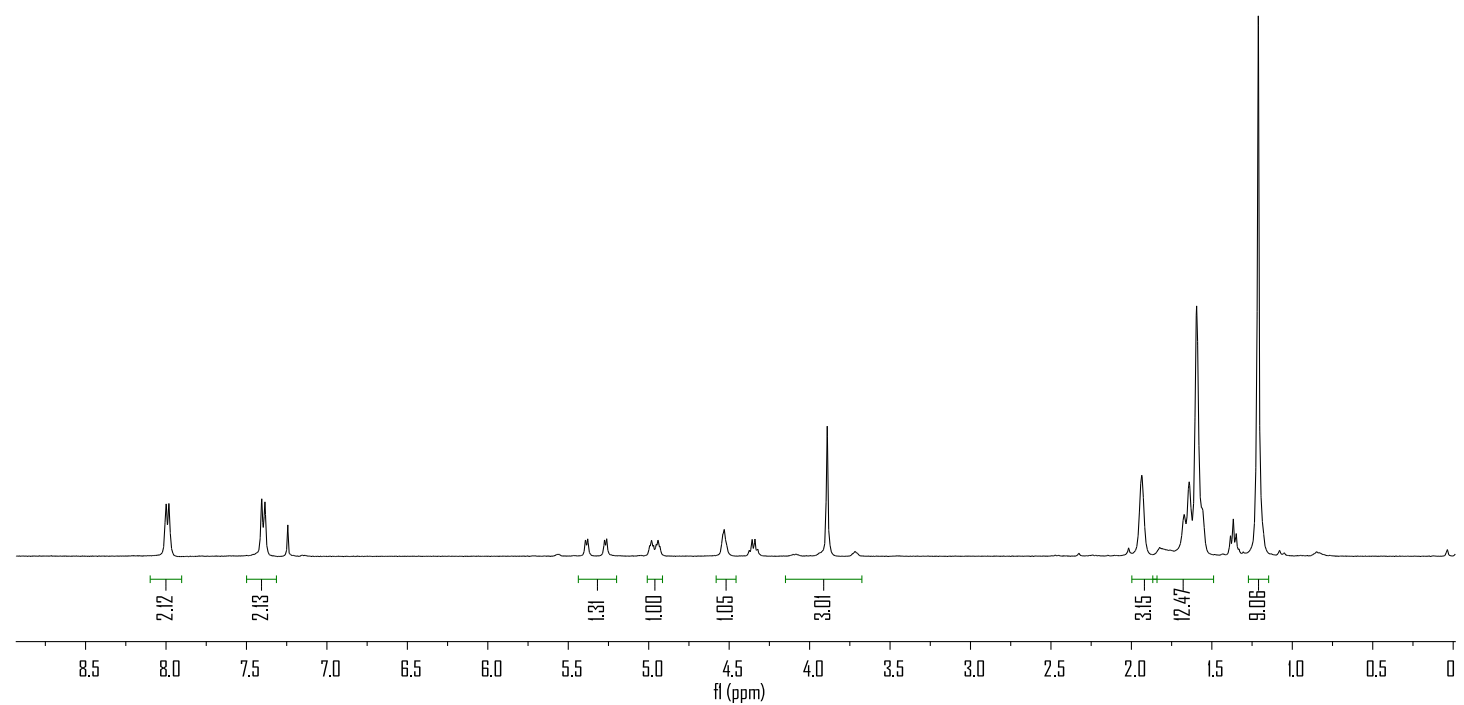


${ }^{19} \mathrm{~F}$ NMR $\left(376 \mathrm{MHz}, \mathrm{CDCl}_{3}\right.$ ) spectrum of $\mathbf{3 h j}$<smiles>CCC(C)(C)S(=O)N[C@H](c1ccc(C(=O)OC)cc1)[C@H](F)C(=O)C12CC3CC(CC(C3)C1)C2</smiles>

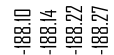

i

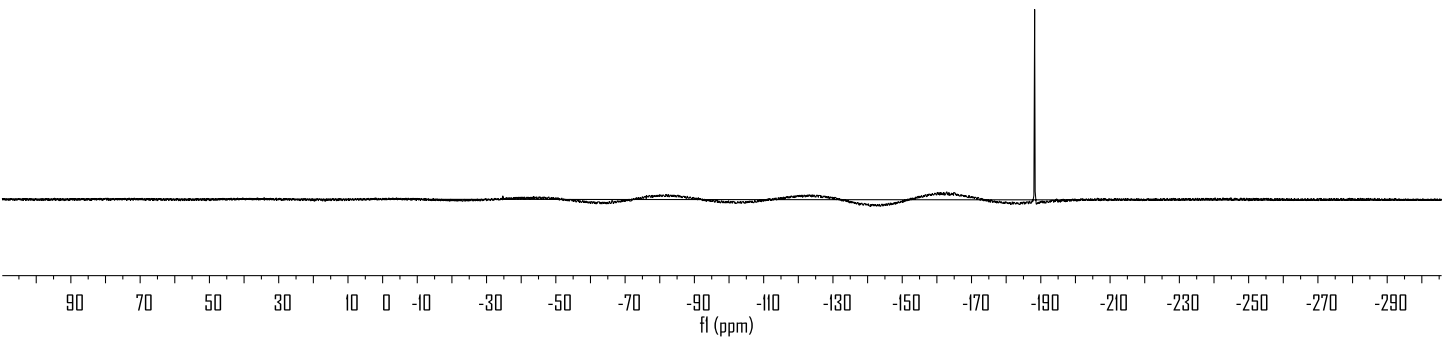


${ }^{13} \mathrm{C}$ NMR (101 MHz, $\left.\mathrm{CDCl}_{3}\right)$ spectrum of $\mathbf{3 h j}$

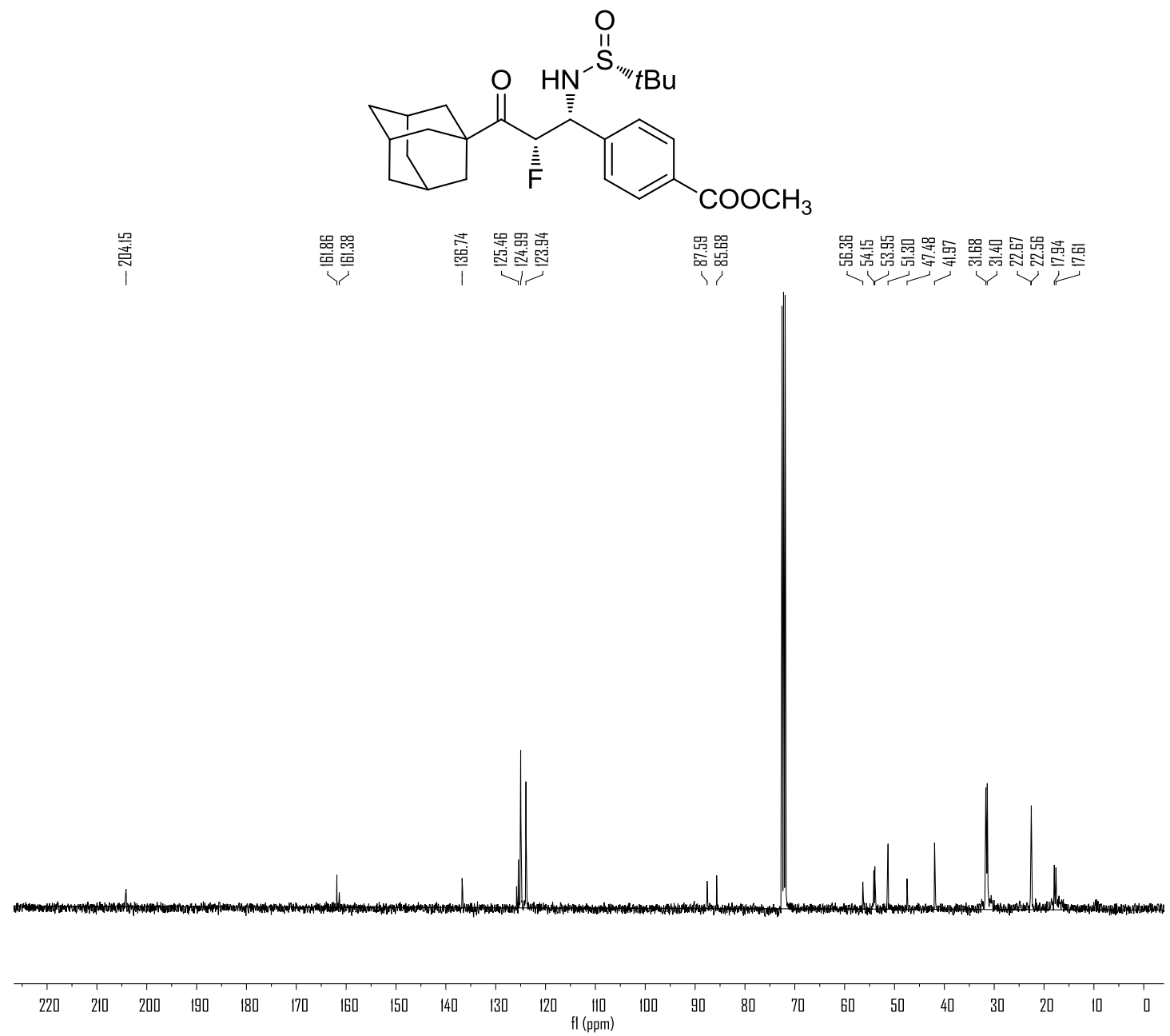


${ }^{19} \mathrm{~F}$ NMR $\left(376 \mathrm{MHz}, \mathrm{CDCl}_{3}\right.$ ) spectrum of $\mathbf{3 h k}$<smiles>O=C([18O])N[C@H](c1ccc([N+](=O)[O-])cc1)C(F)C12CC3CC(CC(C3)C1)C2</smiles>

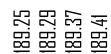

弥

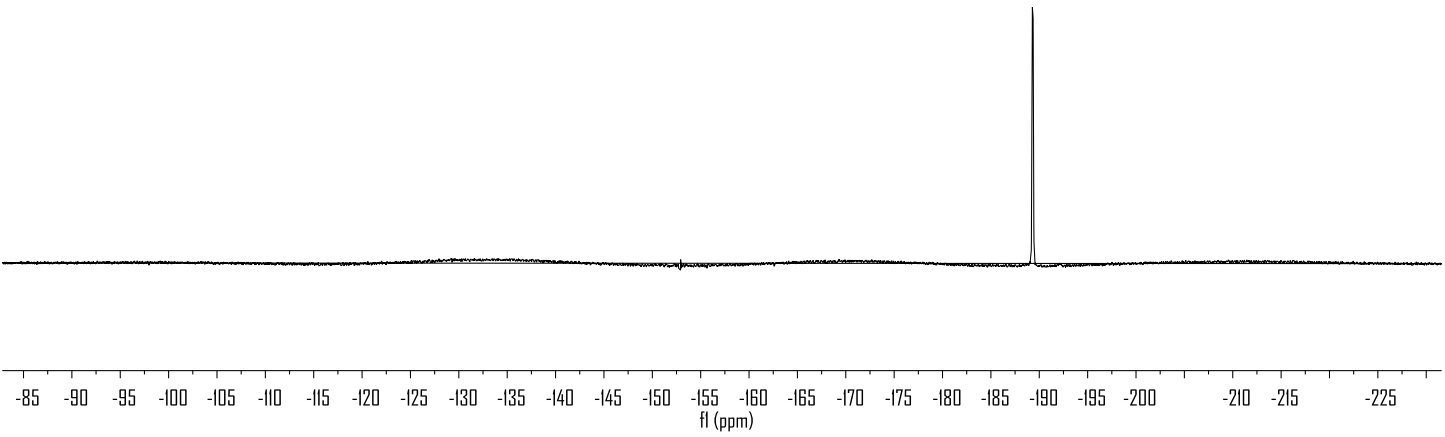


${ }^{13} \mathrm{C}$ NMR $\left(101 \mathrm{MHz}, \mathrm{CDCl}_{3}\right.$ ) spectrum of $\mathbf{3 h k}$<smiles>O=C(N[C@H](c1ccc([N+](=O)[O-])cc1)C(F)C12CC3CC(CC(C3)C1)C2)c1ccccc1</smiles>

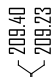

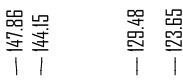

氛牙

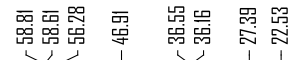

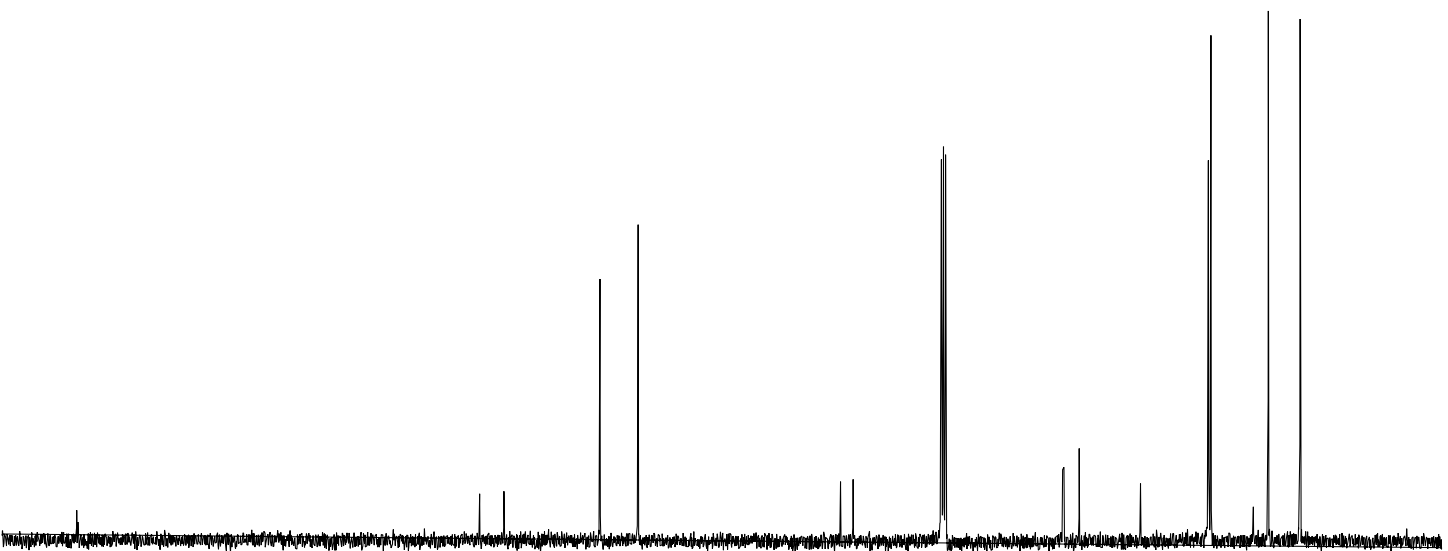

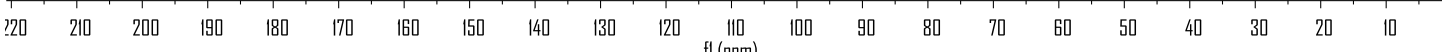


${ }^{1} \mathrm{H}$ NMR (400 MHz, $\mathrm{CDCl}_{3}$ ) spectrum of $\mathbf{3 h l}$<smiles>N#Cc1ccc(C(N[S@](=O)[O-])C(F)C(=O)C23CC4CC(CC(C4)C2)C3)cc1</smiles>

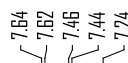

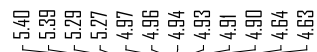

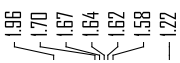

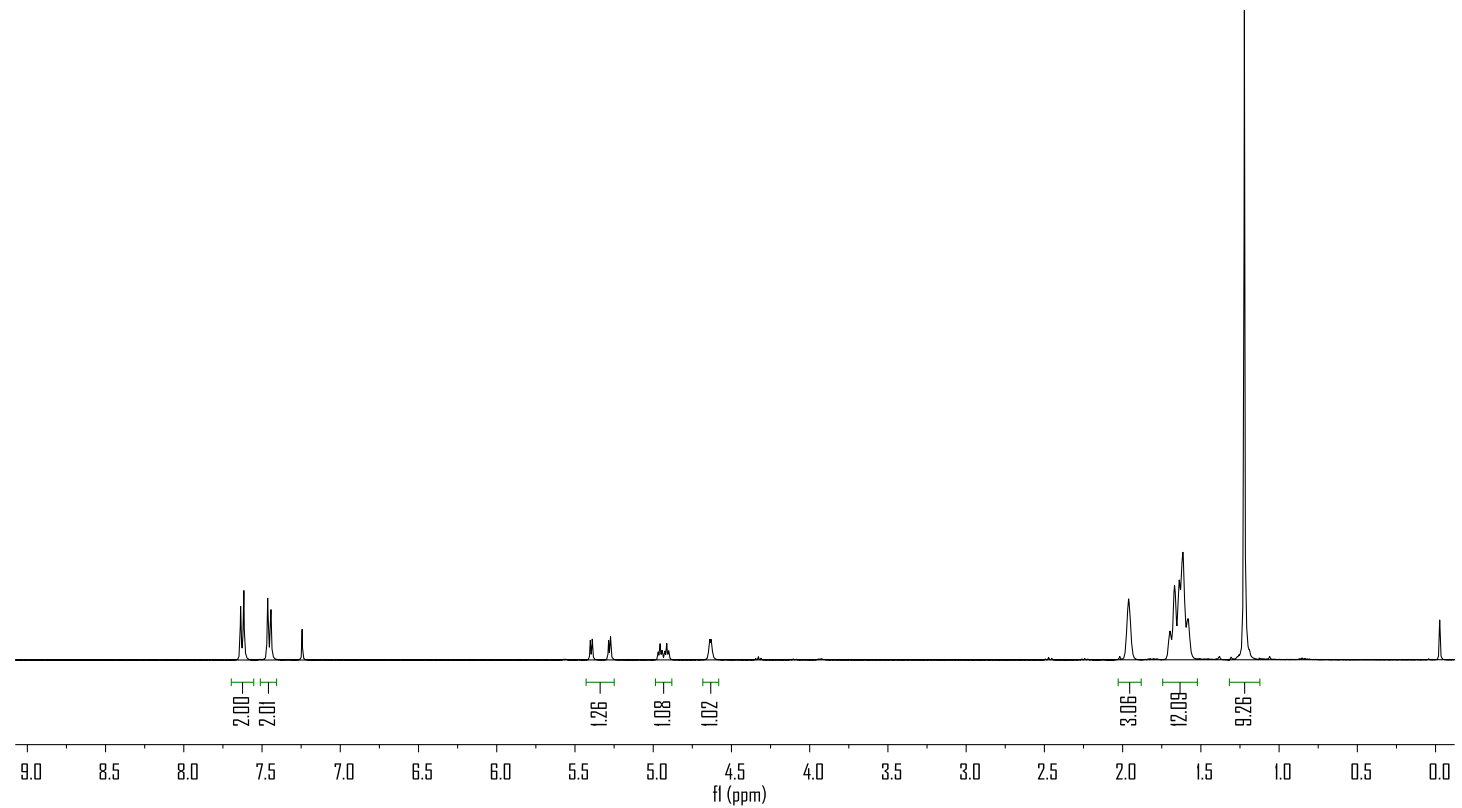


${ }^{19} \mathrm{~F}$ NMR $\left(376 \mathrm{MHz}, \mathrm{CDCl}_{3}\right.$ ) spectrum of $\mathbf{3 h l}$
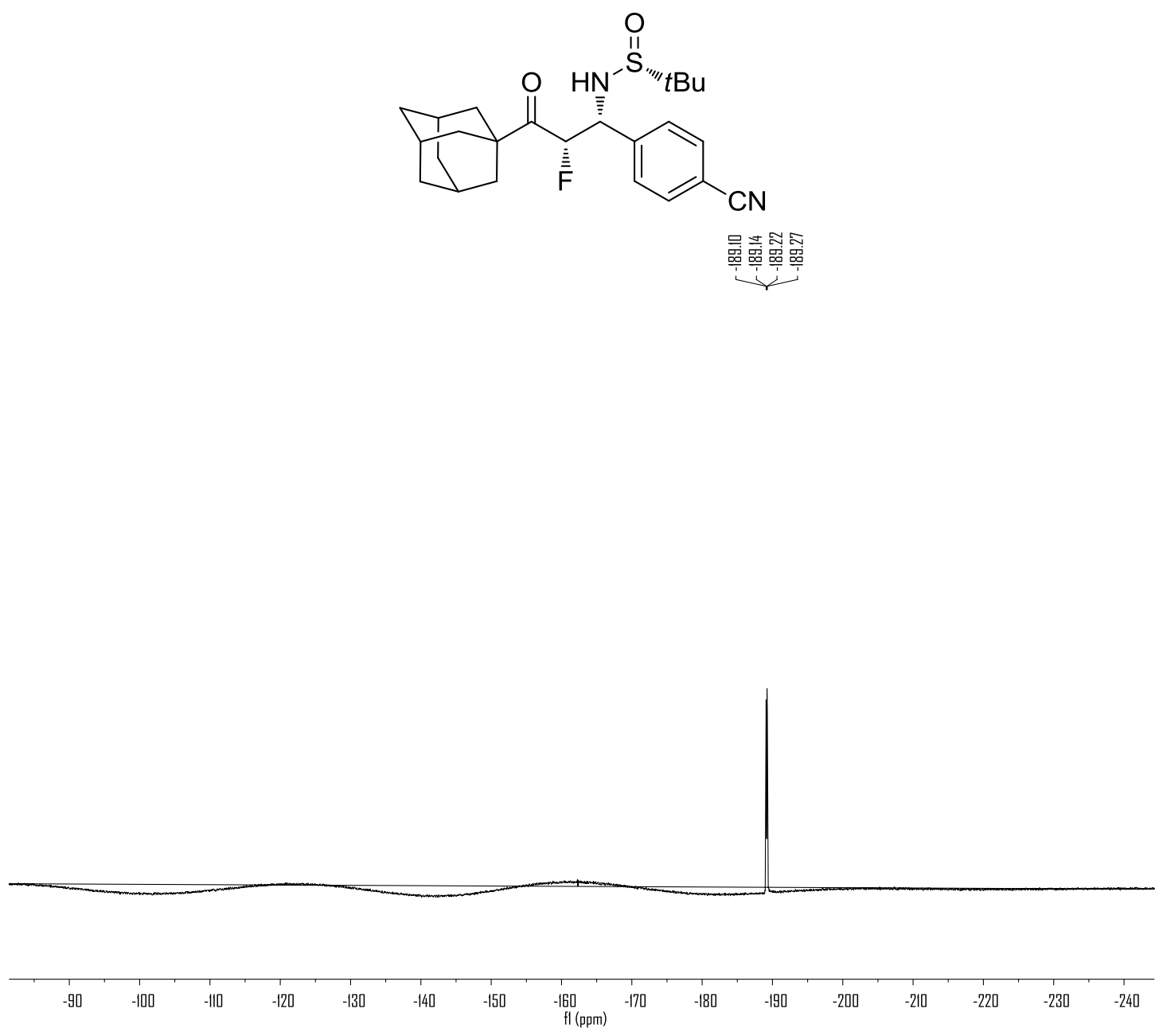

114 
${ }^{13} \mathrm{C}$ NMR (101 MHz, $\mathrm{CDCl}_{3}$ ) spectrum of $\mathbf{3 h l}$<smiles>N#Cc1ccc(C(N[S@](=O)[O-])C(F)C(=O)C23CC4CC(CC(C4)C2)C3)cc1</smiles>

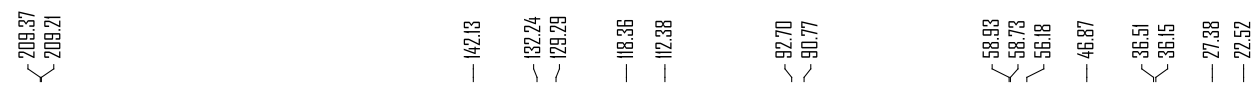

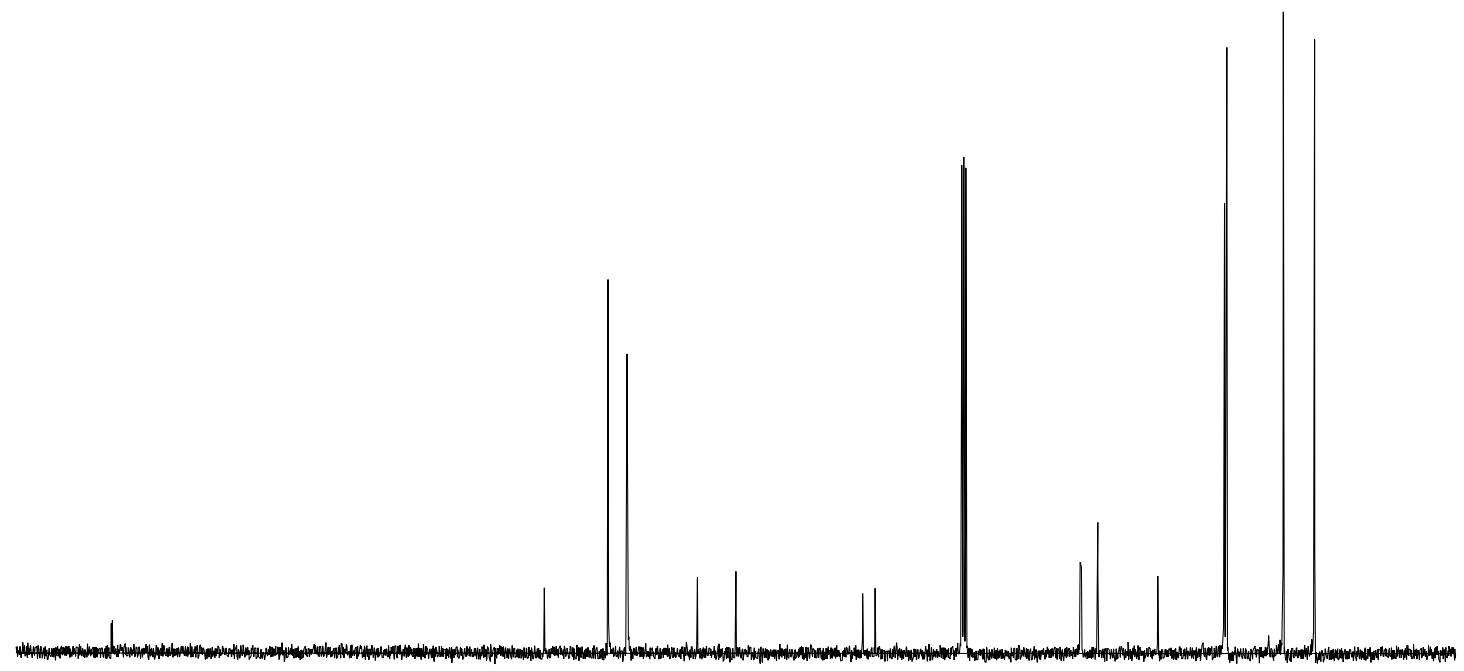

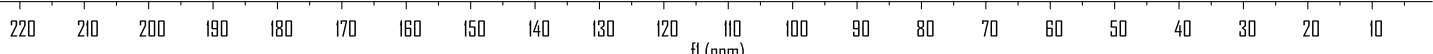


${ }^{1} \mathrm{H}$ NMR (400 MHz, $\mathrm{CDCl}_{3}$ ) spectrum of $\mathbf{3 j i}$<smiles>COc1ccccc1C(=O)C(F)[C@H](NS(=O)C(=O)c1ccccc1)c1ccc(Br)cc1</smiles>

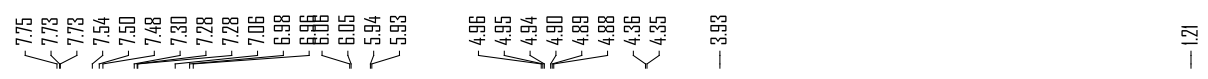

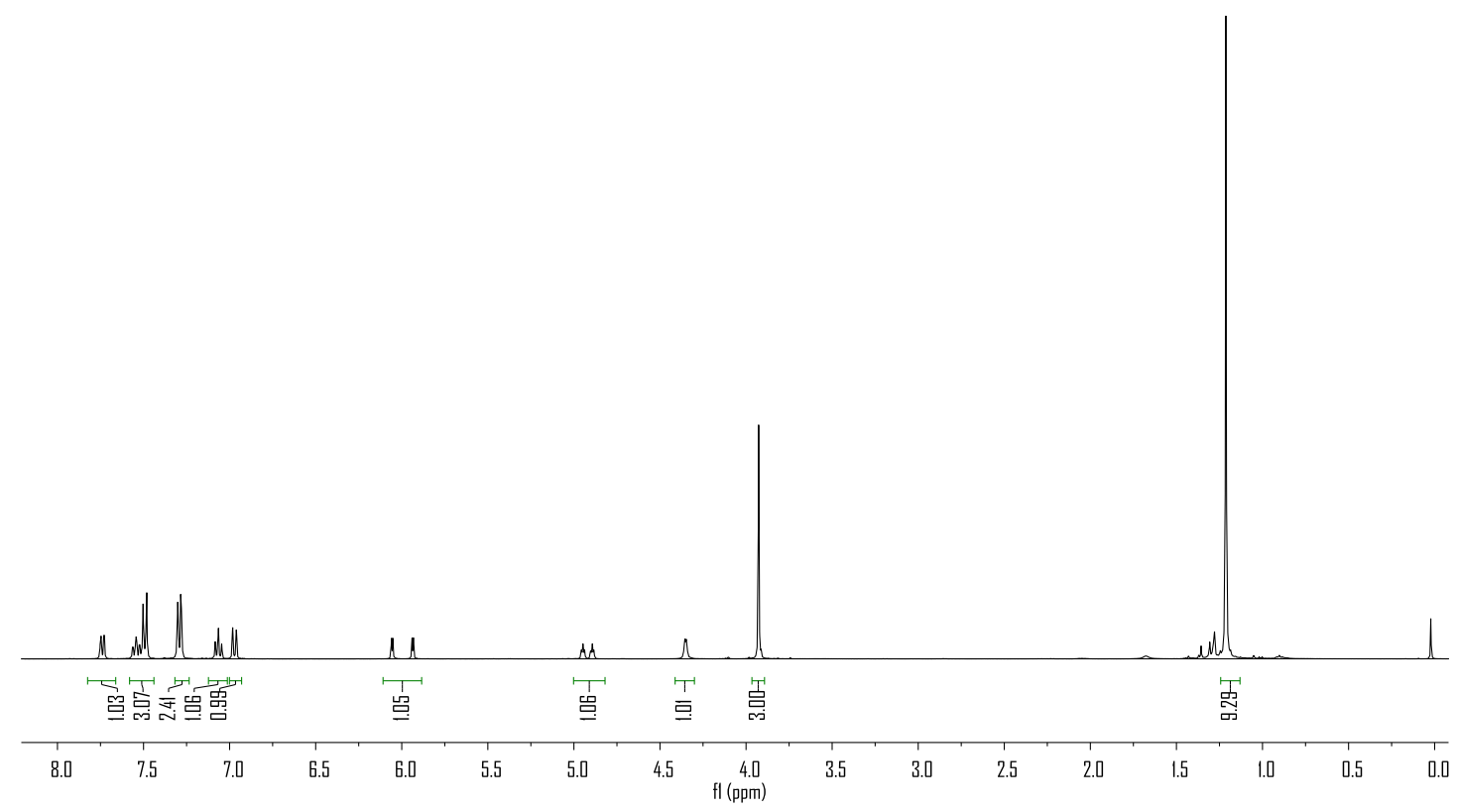


${ }^{19} \mathrm{~F}$ NMR (376 MHz, $\mathrm{CDCl}_{3}$ ) spectrum of $\mathbf{3 j i}$<smiles>COc1ccccc1C(=O)C(F)[C@H](NS(=O)[18O])c1ccc(Br)cc1</smiles>

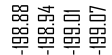

iji

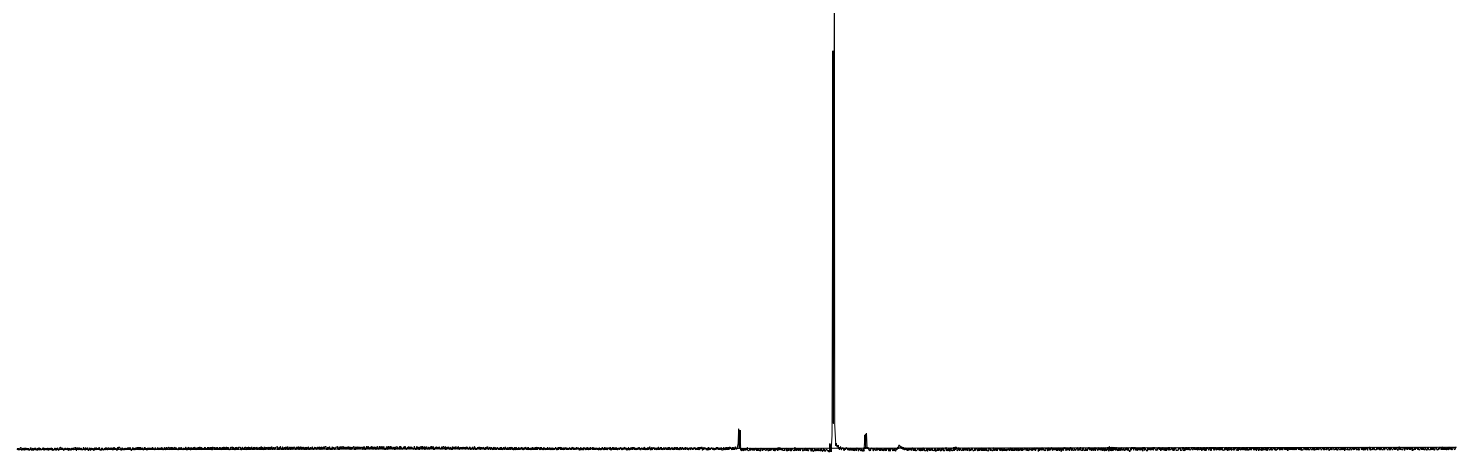

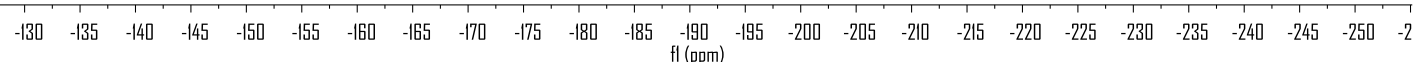


${ }^{13} \mathrm{C}$ NMR (101 MHz, $\mathrm{CDCl}_{3}$ ) spectrum of $\mathbf{3 j i}$<smiles>COc1ccccc1C(=O)C(F)[C@H](NS(=O)[18O])c1ccc(Br)cc1</smiles>

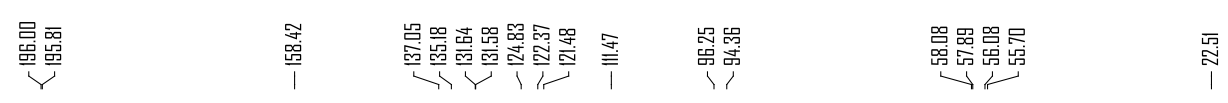

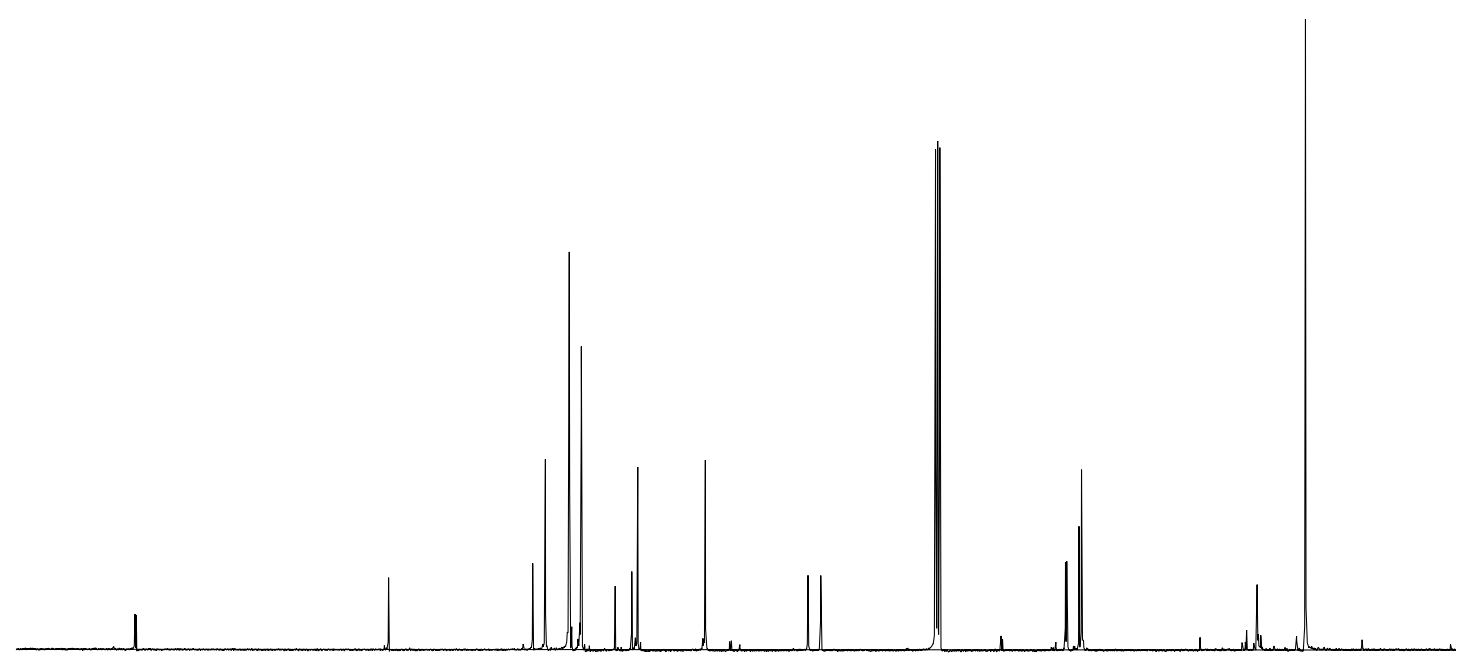

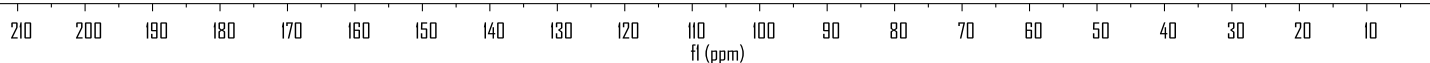


${ }^{1} \mathrm{H} \mathrm{NMR}\left(400 \mathrm{MHz}, \mathrm{CDCl}_{3}\right)$ spectrum of 3na

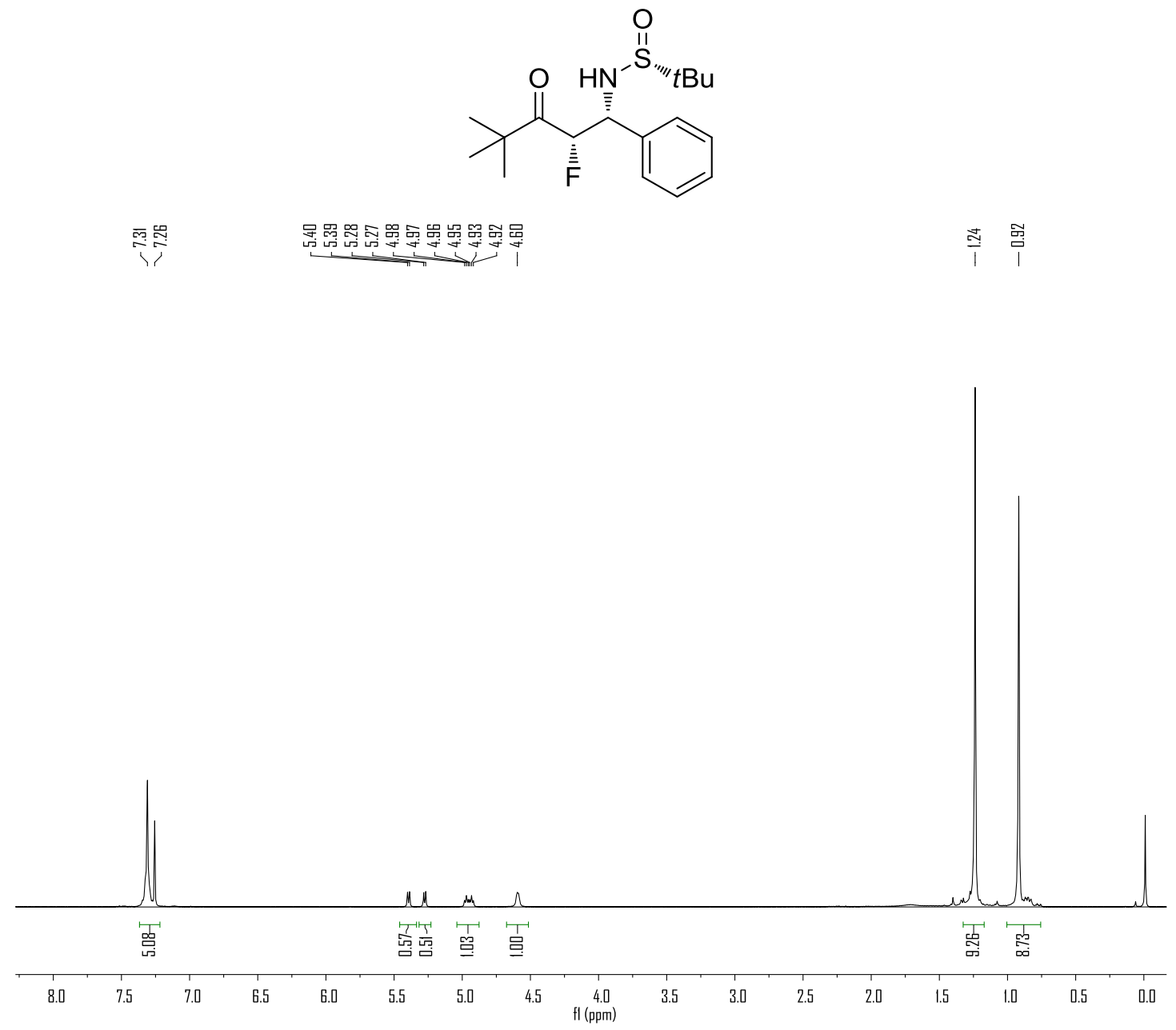


${ }^{19} \mathrm{~F} \mathrm{NMR}\left(376 \mathrm{MHz}, \mathrm{CDCl}_{3}\right.$ ) spectrum of 3na<smiles>CC(C)(C)C(=O)[C@H](F)[C@H](NS(=O)C(C)(C)C)c1ccccc1</smiles>

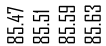

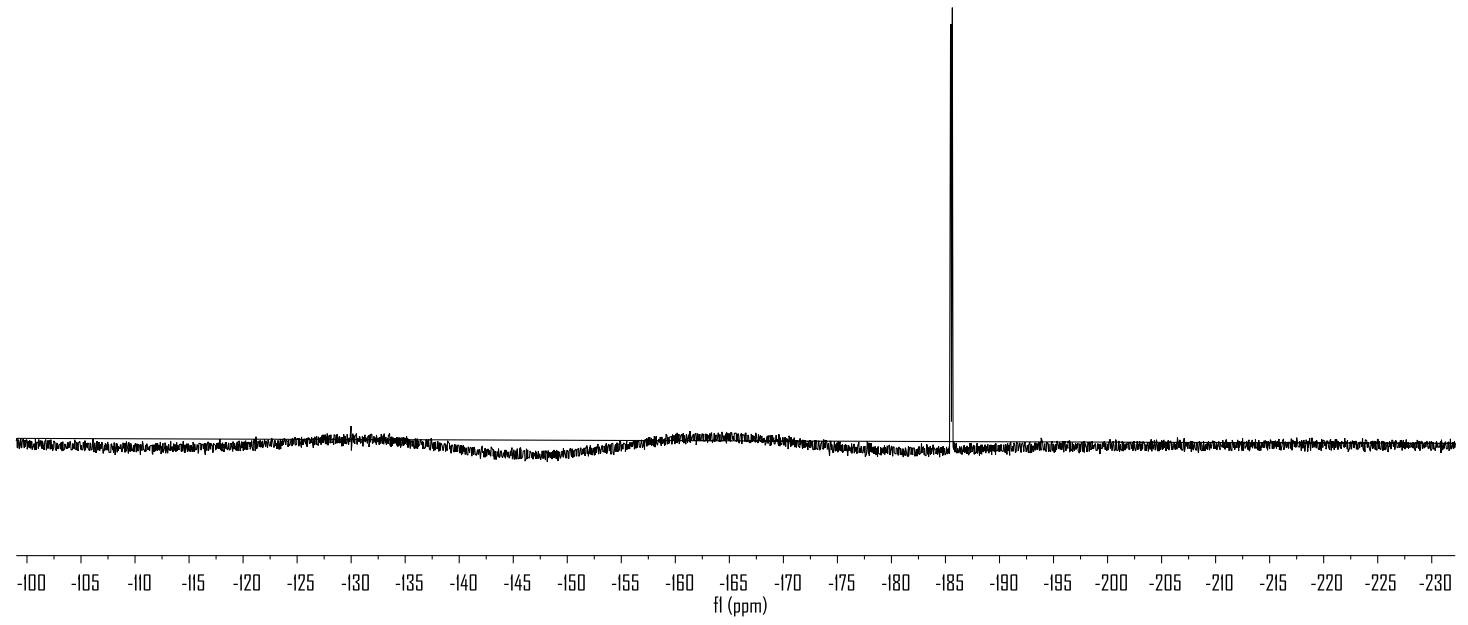


${ }^{13} \mathrm{C}$ NMR (101 MHz, $\mathrm{CDCl}_{3}$ ) spectrum of 3na<smiles>CC(C)(C)C(=O)C(F)[C@H](NS(=O)[O-])c1ccccc1</smiles>

覃昜

ํํำ

初

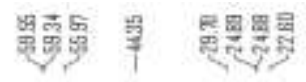

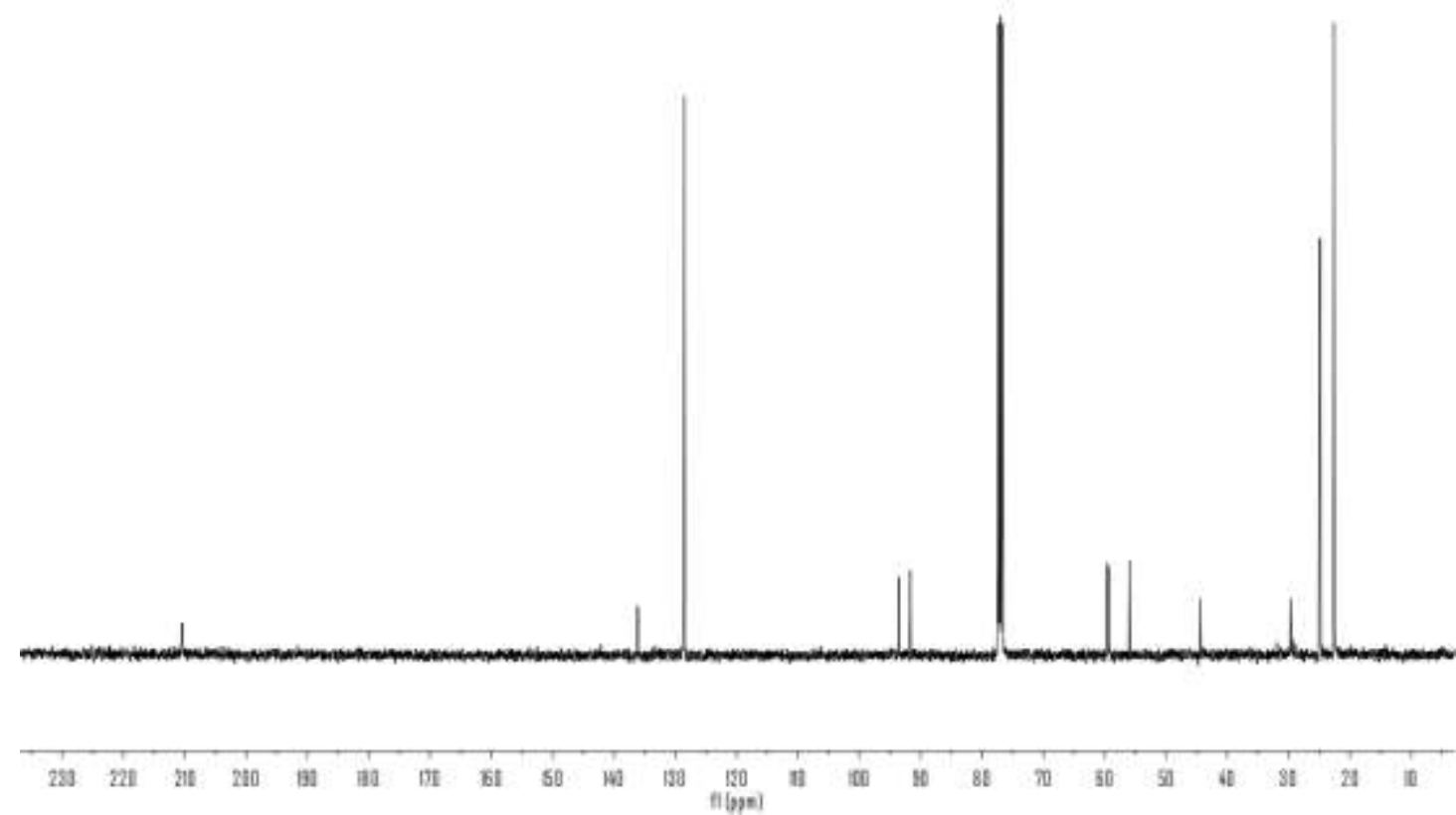


${ }^{1} \mathrm{H}$ NMR (400 MHz, $\mathrm{CDCl}_{3}$ ) spectrum of 4<smiles>CC(C)(C)C(=O)ON[C@H](c1ccc(Br)cc1)C(F)C(=O)C12CC3CC(CC(C3)C1)C2</smiles>

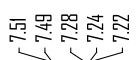

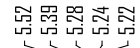

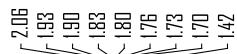

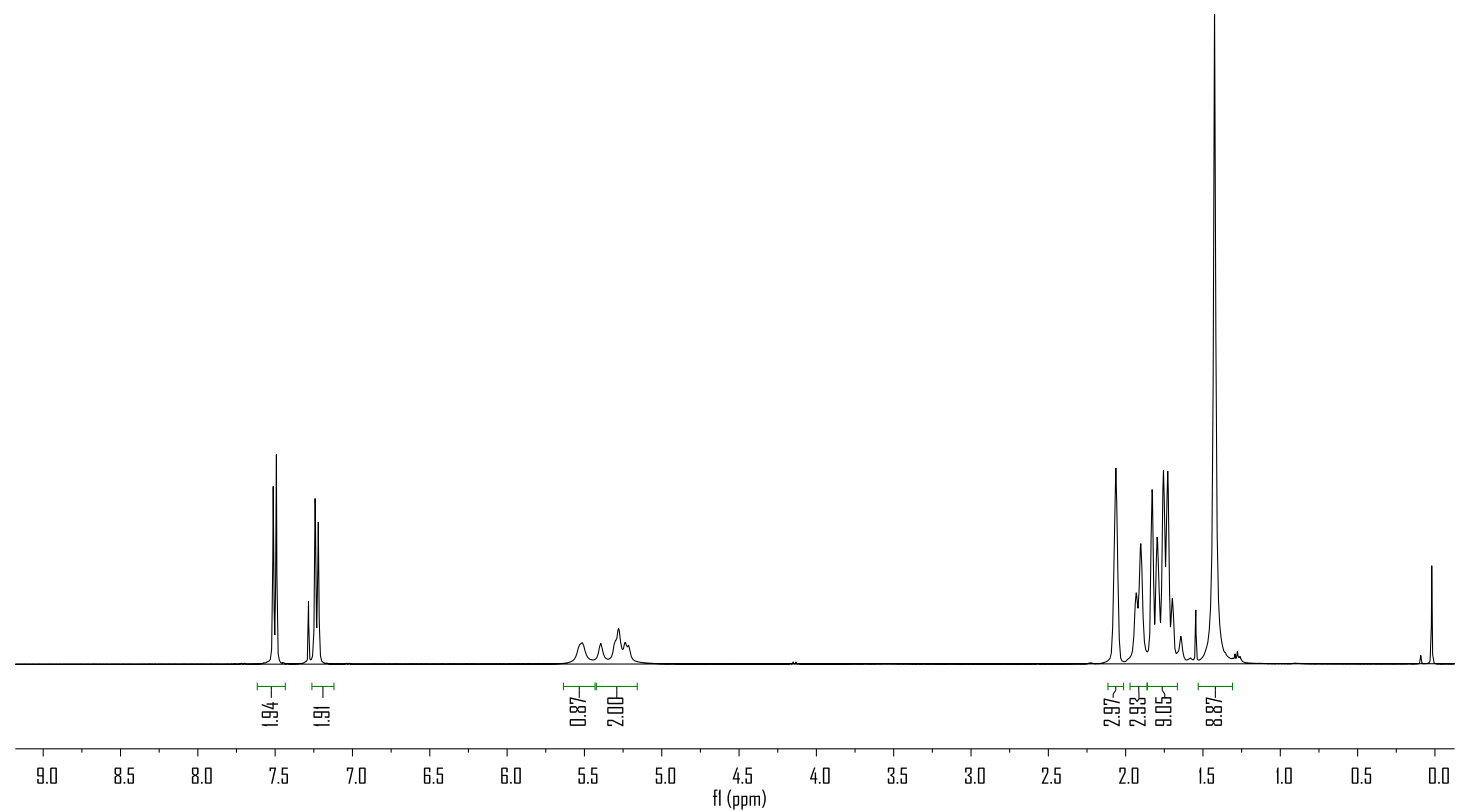


${ }^{19} \mathrm{~F}$ NMR $\left(376 \mathrm{MHz}, \mathrm{CDCl}_{3}\right.$ ) spectrum of 4<smiles>CC(C)(C)C(=O)ON[C@H](c1ccc(Br)cc1)[C@H](F)C(=O)C12CC3CC(CC(C3)C1)C2</smiles>

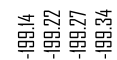

$r$

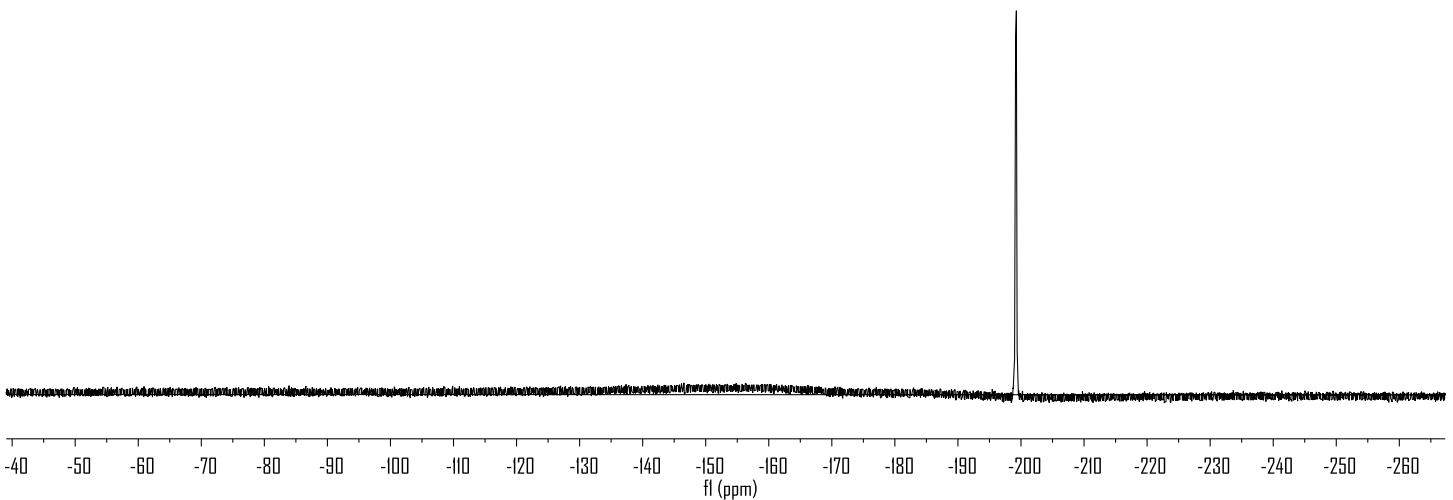


${ }^{13} \mathrm{C}$ NMR (101 MHz, $\mathrm{CDCl}_{3}$ ) spectrum of 4

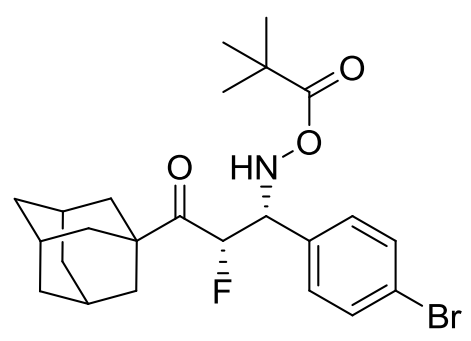

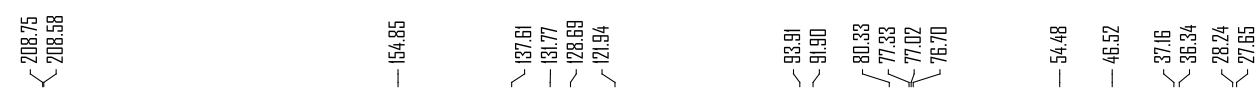

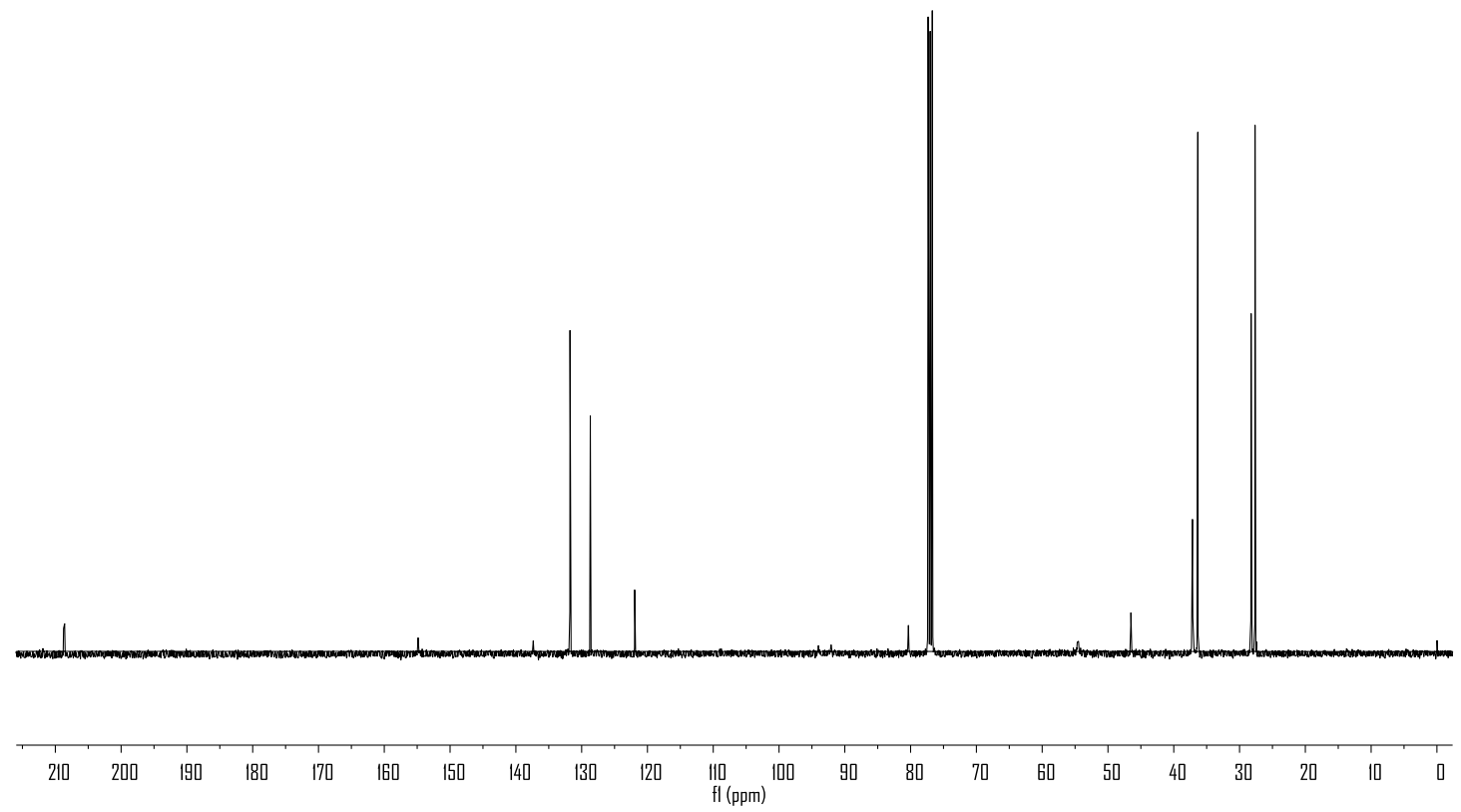

